\title{
On Hecke Algebras Associated with Elliptic Root Systems and the Double Affine Hecke Algebras
}

Dedicated to Masaki Kashiwara on the occasion of his sixtieth birthday

By

Yoshihisa SAIto* and Midori Shiota*

\begin{abstract}
We define the elliptic Hecke algebras for arbitrary marked elliptic root systems in terms of the corresponding elliptic Dynkin diagrams and make a 'dictionary' between the elliptic Hecke algebras and the double affine Hecke algebras.
\end{abstract}

\section{$\S 1$. Introduction}

1.1. Over the last fifteen years or so, there were remarkable developments in the study of multi-variable orthogonal polynomials, attached to root systems. One of these developments was due to Cherednik. In [C1], he defined an difference analogue of Knizhnik-Zamolodikov equations, so-called affine quantum difference Knizhnik-Zamolodikov equations and established their equivalence with the corresponding eigenvalue problem of Macdonald type. To prove the above equivalence, he introduced a new class of algebras, so-called the double affine Hecke algebras. Moreover, he proved Macdonald's inner product conjecture in [C2]. In a process of solving it, the double affine Hecke algebras also played an important role.

Cherednik's construction is generalized to an important class of nonreduced root systems, $\left(C_{n}^{\vee}, C_{n}\right)$ by Noumi $[\mathrm{N}]$ and Sahi [Sa]. When $n=1$

Communicated by M. Kashiwara. Received November 14, 2007. Revised September 1, 2008, December 12, 2008, January 30, 2009.

2000 Mathematics Subject Classification(s): Primary 17B35; Secondary 14D30, 16G20. ${ }^{*}$ Graduate School of Mathematical Sciences, University of Tokyo, Tokyo 153-8914, Japan.

e-mail: yosihisa@ms.u-tokyo.ac.jp

(c) 2009 Research Institute for Mathematical Sciences, Kyoto University. All rights reserved. 
(rank 1 case), the corresponding orthogonal polynomials are the Askey-Wilson polynomial $[\mathrm{AW}]$ which include as special and limiting cases all the classical families of orthogonal polynomials in one variable. In [M5], Macdonald formulated all the above results uniformly.

1.2. In the middle of 1980 's, K. Saito $[\mathrm{S}]$ defined a notion of the marked elliptic root systems which is a generalization of finite or affine root systems, motivated by the study of simple elliptic singularities. Attaching each marked elliptic root system, he introduced a diagram, so-called the elliptic Dynkin diagram which describes the structure of a marked elliptic root system. In addition, he gave a complete classification of marked elliptic root systems under some suitable assumptions. In the original motivation, vertices in an elliptic Dynkin diagram correspond to vanishing cycles and edges describe intersection numbers of them.

After K. Saito's work, he and Takebayashi studied the structure of the Weyl groups associated to marked elliptic root systems, so-called the elliptic Weyl groups [ST]. In particular, they found a new presentation of elliptic Weyl groups in terms of the corresponding elliptic Dynkin diagrams. The explicit meaning is as follows. In the finite and affine cases, it is well-known that the structure of the Weyl groups can be described by the corresponding Coxeter-Dynkin diagrams. Namely, the set of generators and relations of the Weyl group can be read from the corresponding Coxeter-Dynkin diagram. As a generalization, they gave a generating system of the elliptic Weyl group attached to vertices of the elliptic Dynkin diagram and the defining relation which are described by the 'shape' of it. These relations are called the elliptic Coxeter relations.

Since the Weyl groups of finite and affine root systems are Coxeter groups, one can consider the corresponding Hecke algebras. In the elliptic case, as an application of the K. Saito-Takebayashi's presentation, Yamada $[Y]$ defined a $q$-analogue of elliptic Weyl groups called the elliptic Hecke algebras for "onecodimensional" marked elliptic root systems which have only one dotted line in their elliptic Dynkin diagrams. After that Takebayashi [T1], [T2] defined them for arbitrary marked elliptic root systems except for the group (D) (c.f. 4.2). Yamada and Takebayashi also pointed out that elliptic Hecke algebras are much like double affine Hecke algebras. More precisely, for some cases, they stated that the elliptic Hecke algebras are embedded into the double affine Hecke algebras.

1.3. The aim of this article to establish an explicit connection between 
the elliptic Hecke algebras and the double affine Hecke algebras. For that purpose, we reformulate the uniform construction of the double affine Hecke algebras due to Macdonald [M5]. In Section 2, we give a quick review of the theory of the affine Hecke algebras. All statements in this section are wellknown. In Section 3, we introduce a notion of triplets. This is a basic datum to define the double affine Hecke algebras and a key of our construction. For a giving triplet, we define the double affine Hecke algebras and give some basic properties of them. After recalling the theory of elliptic root systems in Section 4 following K. Saito [S], we give the definition of the elliptic Hecke algebras in Section 5. They are defined by some generators and relations attached to the elliptic Dynkin diagrams of the corresponding (marked) elliptic root system. In addition, we give another presentation of them. (Proofs of the statements are given in Section 7.) Section 6 is the main part of this article. For a giving marked elliptic root system $(R, G)$, we introduce the corresponding triplet and the double affine Hecke algebra attached to it as in Section 3. On the other hand we have another algebra (the corresponding elliptic Hecke algebra) attached to $(R, G)$ as in Section 5. After that, we make a comparison between them. This is a main result of this article (Theorems 6.2.3, 6.3.2).

1.4. Finally, we must refer the results of Takebayashi. As we already mentioned above, he introduced a notion of the elliptic Hecke algebras. More precisely, in [T1], he defined them for elliptic root systems of type $(1,1)$ and compare them and the double affine Hecke algebras by case-by-case checking. After that, in [T2], he defined them for arbitrary marked elliptic root systems except for the group (D) (c.f. 4.2), but he did not compare them and the double affine Hecke algebras for arbitrary cases. In his definition, he use new diagrams which are called the "completed elliptic Dynkin diagrams". But, as we mentioned above, the elliptic Dynkin diagram have a concrete meaning in a geometrical setting. Therefore, in this article, we try to 're-define' elliptic Hecke algebras by using the original elliptic Dynkin diagrams, in stead of the completed elliptic Dynkin diagrams and to make an explicit and uniform 'dictionary' between the elliptic Hecke algebras and the double affine Hecke algebras for arbitrary cases.

The announcement of the results of this article already appeared as [SS].

\section{§2. $\quad$ Affine Hecke Algebras}

2.1. Affine root systems and affine Weyl groups. Let $V$ be an $n$ - 
dimensional real vector space with a positive definite symmetric bilinear form $\langle\cdot, \cdot\rangle, R_{0} \subset V$ an irreducible finite root system and fix $a_{1}, \cdots, a_{n}$ simple roots in $R_{0}$. For $a \in R_{0}$ set $a^{\vee}:=2 a /\langle a, a\rangle$. Denote by $Q\left(R_{0}\right)=\oplus \mathbb{Z} a_{i}$ the root lattice, $\left(Q\left(R_{0}\right)\right)_{+}=\oplus \mathbb{Z}_{\geq 0} a_{i}, P\left(R_{0}\right)=\left\{\lambda \in V \mid\left\langle\lambda, a_{i}^{\vee}\right\rangle \in \mathbb{Z}\right\}$ the weight lattice, $\left(P\left(R_{0}\right)\right)_{+}$the set of all dominant weights, $\left(P\left(R_{0}\right)\right)_{-}=-\left(P\left(R_{0}\right)\right)_{+}$and $W\left(R_{0}\right)$ the corresponding Weyl group. Set $R_{0}^{\vee}=\left\{a^{\vee} \mid a \in R_{0}\right\}$. It is also an irreducible finite root system.

Let $\mathbf{F}:=V \oplus \mathbb{R} c$ and we will interpret an element of $\mathbf{F}$ as a function on $V$ by $(u+r c)(v)=\langle u, v\rangle+r$. We extend $\langle\cdot, \cdot\rangle$ to a positive semidefinite bilinear form on $\mathbf{F}$ by $\left\langle u_{1}+r_{1} c, u_{2}+r_{2} c\right\rangle:=\left\langle u_{1}, u_{2}\right\rangle$. Let $S\left(R_{0}\right)$ be the set of all vectors of the form $a+r c$ where $a \in R_{0}$ and $r$ is any integer if $\frac{1}{2} a \notin R_{0}$ (resp. any odd integer if $\left.\frac{1}{2} a \in R_{0}\right)$. Set $a_{0}:=-\theta+c$, where $\theta$ is the highest root of $R_{0}$. Then $S\left(R_{0}\right)$ is an irreducible reduced affine root system with simple roots $a_{0}, a_{1}, \cdots, a_{n}$. We remark that $c$ can be written in the following form: $c=\sum_{i=0}^{n} n_{i} a_{i}$, where $n_{i} \in \mathbb{Z}_{>0}$ and $n_{0}=1$. The dual root system $S\left(R_{0}\right)^{\vee}:=\left\{a^{\vee} \mid a \in S\left(R_{0}\right)\right\}$ is also an irreducible reduced affine root system with a basis $a_{0}^{\vee}, \cdots, a_{n}^{\vee}$.

For later use, we introduce the following notation: set

$$
b_{i}:=\left\{\begin{array}{cl}
a_{i}, & \text { if } S=S\left(R_{0}\right), \\
a_{i}^{\vee}, & \text { if } S=S\left(R_{0}\right)^{\vee} .
\end{array}\right.
$$

For $S=S\left(R_{0}\right)$ or $S\left(R_{0}\right)^{\vee}$, we denote by $Q(S):=\oplus_{i=0}^{n} \mathbb{Z} b_{i}$ its root lattice.

If $R_{0}$ of type $X$ where $X$ is one of the symbols $A_{n}, B_{n}, C_{n}, B C_{n}, D_{n}, E_{6}$, $E_{7}, E_{8}, F_{4}, G_{2}$, we say that $S\left(R_{0}\right)\left(\right.$ resp. $\left.S\left(R_{0}\right)^{\vee}\right)$ is of type $X\left(\right.$ resp. $\left.X^{\vee}\right)$. It is known that any irreducible reduced affine root system $S$ is isomorphic to either $S\left(R_{0}\right)$ or $S\left(R_{0}\right)^{\vee}$. In Appendix, we will present a complete list of irreducible reduced affine root systems.

Firstly assume that $S$ is an irreducible reduced affine root system. Namely $S=S\left(R_{0}\right)$ or $S\left(R_{0}\right)^{\vee}$. Let $W(S)$ be the affine Weyl group of $S$. It is generated by reflections $w_{f}(f \in S)$ where $w_{f}(g)=g-\left\langle g, f^{\vee}\right\rangle f$ for $g \in \mathbf{F}$. Since $\left(f^{\vee}\right)^{\vee}=$ $f$, we have $w_{f} \vee=w_{f}$ and $W\left(S\left(R_{0}\right)\right)=W\left(S\left(R_{0}\right)^{\vee}\right)$. Define the action of $v \in V$ in $\mathbf{F}$ by $t(v): f \mapsto f-\langle f, v\rangle c$. The following fact is well-known.

Theorem 2.1.1. (1) $W(S)=W\left(R_{0}\right) \ltimes t\left(Q\left(R_{0}^{\vee}\right)\right)$. (2) $W(S)$ is generated by $w_{i}:=w_{b_{i}}(i=0, \cdots, n)$ and a Coxeter group which corresponds to the affine Dynkin diagram of $S$.

Let $\tilde{W}(S):=W_{0} \ltimes t\left(P\left(R_{0}^{\vee}\right)\right)$ be the extended affine Weyl group. It is easy to see that $W(S)$ is a normal subgroup of $\tilde{W}(S)$ and $\tilde{W}(S) / W(S) \cong$ 
$P\left(R_{0}^{\vee}\right) / Q\left(R_{0}^{\vee}\right)$. Let $S^{+}$be the set of positive roots and $S^{-}:=-S^{+}$. For $w \in$ $\tilde{W}(S)$, define $l(w):=\left|S^{+} \cap w^{-1} S^{-}\right|$. If $w \in W(S)$, its length with respect to the generators $w_{0}, \cdots, w_{n}$ is just equal to $l(w)$. Define $\Omega:=\{w \in \tilde{W}(S) \mid l(w)=0\}$. It is a subgroup and $\tilde{W}(S)=\Omega \ltimes W(S)$. Therefore $\Omega \cong P\left(R_{0}^{\vee}\right) / Q\left(R_{0}^{\vee}\right)$. Since $\Omega$ is a subgroup of $\tilde{W}(S)$, it acts on $S$. Moreover, it is known that, $\Omega$ preserves the set of all simple roots. Therefore, for $u \in \Omega$ such that $u\left(b_{i}\right)=b_{j}$, we have $u w_{i} u^{-1}=w_{j}$.

For later use, we will explain an explicit structure of $\Omega$. Let $v_{i}$ be the shortest element of $W\left(R_{0}\right)$ such that $v_{i} \omega_{i} \in P\left(R_{0}^{\vee}\right)_{-}$, where $\left\{\omega_{i}\right\}_{i=1}^{n}$ is the set of all fundamental weights of $P\left(R_{0}^{\vee}\right)$. Let $u_{i}=t\left(\omega_{i}\right) v_{i}^{-1}(1 \leq i \leq n)$ and $u_{0}=1$.

Lemma 2.1.2. Set $J=\left\{j \mid 0 \leq j \leq n, n_{j}=1\right\}$. We have $\Omega=$ $\left\{u_{j} \mid j \in J\right\}$.

Remark. We have already defined $W(S)$ and $\tilde{W}(S)$ for any irreducible reduced affine root system (not only for $S=S\left(R_{0}\right)$ ). As we mentioned above, $W\left(S\left(R_{0}\right)\right)=W\left(S\left(R_{0}\right)^{\vee}\right)$. Moreover, by the construction, we have $\tilde{W}\left(S\left(R_{0}\right)\right)=$ $\tilde{W}\left(S\left(R_{0}\right)^{\vee}\right)$.

Secondly assume $S$ is an irreducible, non-reduced affine root system. In this case, the following fact is known:

Fact 1. Let $S_{1}:=\{a \in S \mid a / 2 \notin S\}$ and $S_{2}:=\{a \in S \mid 2 a \notin S\}$. We have $S=S_{1} \cup S_{2}$ and both $S_{1}$ and $S_{2}$ are reduced affine root systems with the same affine Weyl group.

We say that $S$ is of type $\left(X_{1}, X_{2}\right)$ where $X_{i}$ is the type of $S_{i}(i=1,2)$. In this case, the basis of $S$ is that of $S_{1}$ and its affine Weyl group $W(S)$ is equal to $W\left(S_{1}\right)=W\left(S_{2}\right)$

2.2. Affine Hecke algebras. In this subsection, we assume $S$ is reduced.

Definition 2.2.1. (1) Let $\tilde{\mathcal{B}}$ the group with generators $T(w)(w \in \tilde{W}(S))$ and relations:

$$
T(v) T(w)=T(v w), \quad \text { if } l(v)+l(w)=l(v w) .
$$

(2) Let $\mathcal{B}$ be the subgroup of $\tilde{\mathcal{B}}$ generated by $T_{i}:=T\left(w_{i}\right)(i=0, \cdots, n)$.

We write $U_{j}=T\left(u_{j}\right)$ for $j \in J$. It is known that $\tilde{\mathcal{B}}$ is generated by $T_{i}, U_{j}$. 
Consider a Laurent polynomial ring $\mathbb{Z}\left[\tau_{0}^{ \pm 1}, \cdots, \tau_{n}^{ \pm 1}\right]$. Let $\tilde{\mathcal{I}}$ (resp. $\mathcal{I}$ ) be the ideal generated by the elements $\tau_{i}-\tau_{j}$ where $w_{i}$ and $w_{j}$ are conjugate in $\tilde{W}(S)($ resp. $W(S))$. Set

$$
\tilde{\mathcal{A}}_{a}=\mathbb{Z}\left[\tau_{0}^{ \pm 1}, \cdots, \tau_{n}^{ \pm 1}\right] / \tilde{\mathcal{I}} \quad \text { and } \quad \mathcal{A}_{a}=\mathbb{Z}\left[\tau_{0}^{ \pm 1}, \cdots, \tau_{n}^{ \pm 1}\right] / \mathcal{I} .
$$

Obviously both $\tilde{\mathcal{A}}_{a}$ and $\mathcal{A}_{a}$ are isomorphic to some Laurent polynomial rings in several variables. More precisely, if $R_{0}$ is simply laced, $\tilde{\mathcal{A}}_{a}$ is a Laurent polynomial ring in one variable. If not, $\tilde{\mathcal{A}}_{a}$ have two variables; one corresponds to short roots and the other to long roots. If $R_{0}$ is not of type $A_{1}$ or $C_{n}, \tilde{\mathcal{I}}=\mathcal{I}$. Therefore we have $\tilde{\mathcal{A}}_{a}=\mathcal{A}_{a}$. In the case of type $A_{1}, \mathcal{A}_{a}$ has two variables. These are $\tau_{0}, \tau_{1}$ which correspond to simple roots $a_{0}$ and $a_{1}$. In the case of type $C_{n}, \mathcal{A}_{a}$ has three variables. These are $\tau_{0}, \tau_{n}$ and $\tau_{1}=\cdots=\tau_{n-1}$. Here $a_{0}$ and $a_{n}$ are long simple roots and the others are short simple roots.

Definition 2.2.2. (1) The extended affine Hecke algebra $\mathcal{H}(\tilde{W}(S))$ is the quotient of the group algebra $\tilde{\mathcal{A}}_{a}[\mathcal{B}]$ by the ideal generated by the following relations:

$$
\left(T_{i}-\tau_{i}\right)\left(T_{i}+\tau_{i}^{-1}\right)=0, \quad \text { for } i=0, \cdots, n .
$$

(2) The affine Hecke algebra $\mathcal{H}(W(S))$ is the quotient of the group algebra $\mathcal{A}_{a}[\mathcal{B}]$ by the ideal generated by the same relations as (A1).

We regard $\tilde{\mathcal{A}}_{a}$ as an $\mathcal{A}_{a^{-}}$algebra via a natural projection $\mathcal{A}_{a} \rightarrow \tilde{\mathcal{A}}_{a}$. The $\tilde{\mathcal{A}}_{a^{-}}$ algebra $\tilde{\mathcal{A}}_{a} \otimes_{\mathcal{A}_{a}} \mathcal{H}(W(S))$ is naturally isomorphic to the subalgebra of $\mathcal{H}(\tilde{W}(S))$ which is generated by $T_{i}(i=0, \cdots, n)$.

Theorem 2.2.3. $\quad$ Under the convention which we mentioned above, we have $\mathcal{H}(\tilde{W}(S))=\Omega \ltimes\left(\tilde{\mathcal{A}}_{a} \otimes_{\mathcal{A}_{a}} \mathcal{H}(W(S))\right)$, where the action of $\Omega$ on $T_{i}$ is the same as Weyl group case.

There is another presentation of $\mathcal{H}(\tilde{W}(S))$ which is very useful to study affine Hecke algebras. For $\mu^{\prime} \in P\left(R_{0}^{\vee}\right)$ define $Y^{\mu^{\prime}} \in \mathcal{H}(\tilde{W}(S))$ as follows: (i) If $\mu^{\prime} \in P\left(R_{0}^{\vee}\right)_{+}$, then $Y^{\mu^{\prime}}:=T\left(t\left(\mu^{\prime}\right)\right)$; (ii) If $\mu^{\prime}=\lambda^{\prime}-\nu^{\prime}$ with $\lambda^{\prime}, \nu^{\prime} \in P\left(R_{0}^{\vee}\right)_{+}$, then $Y^{\mu^{\prime}}:=T\left(t\left(\lambda^{\prime}\right)\right) T\left(t\left(\nu^{\prime}\right)\right)^{-1}$.

For $\mathcal{H}(W(S))$, we also define $Y^{\mu^{\prime}} \in \mathcal{H}(W(S))$ in the similar way by replacing $P\left(R_{0}^{\vee}\right)$ with $Q\left(R_{0}^{\vee}\right)$.

We introduce the following notation: let

$$
\mathbf{b}\left(z_{1}, z_{2} ; x\right)=\frac{z_{1}-z_{1}^{-1}+\left(z_{2}-z_{2}^{-1}\right) x}{1-x^{2}},
$$


where $z_{1}, z_{2}$ and $x$ are indeterminates. When $z_{1}=z_{2}, \mathbf{b}\left(z_{1}, z_{2} ; x\right)$ has the simpler form

$$
\mathbf{b}\left(z_{1}, z_{1} ; x\right)=\frac{z_{1}-z_{1}^{-1}}{1-x} .
$$

In the case of $\mathcal{H}(\tilde{W}(S))$ such that $R_{0}$ is of type $A_{1}$ or of type $C_{n}$, for $1 \leq i \leq n$, we set

$$
\tau_{i}^{\prime}= \begin{cases}\tau_{i}, & i \neq n \\ \tau_{0}, & i=n\end{cases}
$$

For the other case, we set $\tau_{i}^{\prime}=\tau_{i}$ for any $i=1, \cdots, n$.

Theorem 2.2.4. (1) $Y^{\mu^{\prime}}$ is well-defined for all $\mu^{\prime}$ and

$$
Y^{\mu^{\prime}} Y^{\nu^{\prime}}=Y^{\mu^{\prime}+\nu^{\prime}} .
$$

(2) In the algebra $\mathcal{H}(\tilde{W}(S))$ (resp. $\mathcal{H}(W(S))$ ), the following relations hold (called Lusztig's relations) :

$$
Y^{\mu^{\prime}} T_{i}-T_{i} Y^{w_{i}\left(\mu^{\prime}\right)}=\mathbf{b}\left(\tau_{i}, \tau_{i}^{\prime} ; Y^{-a_{i}^{\vee}}\right)\left(Y^{\mu^{\prime}}-Y^{w_{i}\left(\mu^{\prime}\right)}\right),
$$

for $i=1, \cdots, n$ and $\mu^{\prime} \in P\left(R_{0}^{\vee}\right)\left(\right.$ resp. $\left.Q\left(R_{0}^{\vee}\right)\right)$.

(3) Let us consider the algebra generated by $T_{i}(i=1, \cdots, n)$ and $Y^{\mu^{\prime}}\left(\mu^{\prime} \in\right.$ $\left.P\left(R_{0}^{\vee}\right)\right)$ and relations $(A 1)$ for $i=1, \cdots, n,(A 2)$ and $(A 3)$. Then it is isomorphic to $\mathcal{H}(\tilde{W}(S))$. Further, by replacing $P\left(R_{0}^{\vee}\right)$ with $Q\left(R_{0}^{\vee}\right)$, the corresponding algebra is isomorphic to $\mathcal{H}(W(S))$.

\section{§3. Double Affine Hecke Algebras}

In this section, we give the definition of the double affine Hecke algebras in terms of triplets. We remark that our definition is not new. It is only a reformulation of the uniform construction of the double affine Hecke algebras due to Macdonald [M5].

3.1. Triplets. Let us consider the following three types of datum $\Xi=\left(R_{0} ; S\right.$, $\Lambda_{s}$ ) which we call a triplet:

(type I) $\quad R_{0}$ is a finite irreducible reduced root system,

$$
S=S\left(R_{0}\right) \text { or } S\left(R_{0}\right)^{\vee}, \quad \Lambda_{s}=Q\left(S^{\prime}\right) \text { where } S^{\prime}=S\left(R_{0}\right) .
$$

(type II)

$R_{0}$ and $S$ are as the same in type I, $\Lambda_{s}=Q\left(S^{\prime}\right)$ where $S^{\prime}=S\left(R_{0}\right)^{\vee}$. 
(type III) $\quad R_{0}$ is of type $C_{n}(n \geq 1)$ (Here we denote $\left.C_{1}=A_{1}.\right)$;

$$
S \text { is of type }\left(C_{n}^{\vee}, C_{n}\right), \Lambda_{s}=Q\left(S\left(R_{0}\right)^{\vee}\right) .
$$

(In Appendix, we present the detailed structure of the affine root system of type $\left(C_{n}^{\vee}, C_{n}\right)$.)

Set

$$
L=\left\{\begin{array}{ll}
P\left(R_{0}\right), & (\text { type I }), \\
P\left(R_{0}^{\vee}\right), & (\text { type II), } \\
Q\left(R_{0}^{\vee}\right), & (\text { type III }),
\end{array} \quad L^{\prime}= \begin{cases}P\left(R_{0}^{\vee}\right), & (\text { type I), } \\
P\left(R_{0}^{\vee}\right), & \text { (type II), } \\
Q\left(R_{0}^{\vee}\right), & \text { (type III) }\end{cases}\right.
$$

In each case, let $\Lambda:=L \oplus \mathbb{Z} c_{0}$. Here $c_{0}=e^{-1} c$ and $e$ is the exponent of $\Omega \cong P\left(R_{0}^{\vee}\right) / Q\left(R_{0}^{\vee}\right)$

Next we fix a normalization of $\langle\cdot, \cdot\rangle$. Recall a basis $\left\{a_{i}\right\}_{i=0}^{n}$ of $S\left(R_{0}\right)$. For type I and II, we normalize $\langle\cdot, \cdot\rangle$ as $\langle\theta, \theta\rangle=2$. Therefore we have $a_{0}^{\vee}=-\theta+c=$ $a_{0}$. For type III, $S=S_{1} \cup S_{2}$ where $S_{1}=S\left(R_{0}\right)^{\vee}$ and $S_{2}=S\left(R_{0}\right)$. Here $R_{0}$ is of type $C_{n}(n \geq 1)$. In this case $e=2$ and we normalize $\langle\cdot, \cdot \cdot\rangle$ as $\langle\theta, \theta\rangle=4$. Therefore we have $\left\langle a_{0}, a_{0}\right\rangle=\left\langle a_{n}, a_{n}\right\rangle=4$ and $\left\langle a_{i}, a_{i}\right\rangle=2(i=1, \cdots, n-1)$. Moreover a basis $\left\{a_{i}^{\vee}\right\}$ of $S_{1}$ and a basis $\left\{a_{i}\right\}$ of $S_{2}$ are related by the following way:

$$
a_{0}^{\vee}=\frac{1}{2} a_{0}=-\frac{\theta}{2}+\frac{c}{2}, \quad a_{n}^{\vee}=\frac{1}{2} a_{n}, \quad a_{i}^{\vee}=a_{i}(i=1, \cdots, n-1) .
$$

Under the above convention, we immediately have the following lemma.

Lemma 3.1.1. For any case, $\Lambda_{s}$ is a sublattice of $\Lambda$.

Let us introduce the following notations:

$$
a_{i}^{\sharp}:=\left\{\begin{array}{cl}
a_{i}, & (\text { type I) } \\
a_{i}^{\vee}, & (\text { type II or III). }
\end{array}\right.
$$

We remark that $\left\{a_{i}^{\sharp}\right\}_{i=0}^{n}$ is a basis of $\Lambda_{s}$.

In each case, let

$$
W(\Xi):=W\left(R_{0}\right) \ltimes t\left(L^{\prime}\right), \quad \text { and } \quad W(\Xi)_{s}:=W\left(R_{0}\right) \ltimes t\left(Q\left(R_{0}^{\vee}\right)\right) .
$$

If $\Xi$ is of type I or II, the first one is the extended affine Weyl group of $S$ and the second is non-extended one. On the other hand, for type III, both are the 
affine Weyl group of $S$.

The first statement of the following lemma is due to Macdonald [M5] and the second is trivial by the definition.

Lemma 3.1.2. (1) $\Lambda$ is stable under the action of $W(\Xi)$.

(2) $\Lambda_{s}$ is stable under the action of $W(\Xi)_{s}$.

3.2. Definition of double affine Hecke algebras. Let $\mathcal{A}$ be a commutative ring defined by the following way:

$$
\mathcal{A}= \begin{cases}\tilde{\mathcal{A}}_{a}, & \text { (type I or II), } \\ \mathcal{A}_{a}\left[\left(\tau_{0}^{\natural}\right)^{ \pm 1},\left(\tau_{n}^{\natural}\right)^{ \pm 1}\right], & \text { (type III), }\end{cases}
$$

where $\tau_{0}^{\natural}$ and $\tau_{n}^{\natural}$ are new indeterminates.

Definition 3.2.1. Let $\Xi=\left(R_{0} ; S, \Lambda_{s}\right)$ be a triplet given in the previous subsection. For $i=0, \cdots, n$, let $\mathbf{b}_{i}(x)=\mathbf{b}\left(\tau_{i}, \tau_{i}^{\natural} ; x\right)$. Here we set $\tau_{i}^{\natural}=\tau_{i}$ for all $i$ when $\Xi$ is of type I or II and for $i \neq 0, n$ when $\Xi$ is of type III.

The double affine Hecke algebra $\mathcal{H}(\Xi)$ is an associative $\mathcal{A}$-algebra defined by the following way.

If $\Xi$ is of type I or II, it is generated by $T_{i}(i=0, \cdots, n), U_{j}(j \in J)$, $X^{\lambda}(\lambda \in \Lambda)$ subject to the following relations:

$$
T_{i} \text { and } U_{j} \text { satisfy the same relations in } \mathcal{H}(W(S)) \text {, }
$$

$$
\begin{gathered}
X^{\lambda} X^{\mu}=X^{\mu} X^{\lambda}=X^{\lambda+\mu}, \\
T_{i} X^{\lambda}-X^{w_{i}(\lambda)} T_{i}=\mathbf{b}_{i}\left(X^{a_{i}^{\sharp}}\right)\left(X^{\lambda}-X^{w_{i}(\lambda)}\right), \\
U_{j} X^{\lambda} U_{j}^{-1}=X^{u_{j}(\lambda)} .
\end{gathered}
$$

If $\Xi$ is of type III, it is generated by $T_{i}(i=0, \cdots, n), X^{\lambda}(\lambda \in \Lambda)$ subject to the similar relations as (D1), (D2), (D3).

Following [M5], we say $\mathcal{H}(\Xi)$ is the double affine Hecke algebra of type $\left(S, S^{\prime}\right)$ for a triplet $\Xi$ of type I or II. For a triplet of type III, we say $\mathcal{H}(\Xi)$ is the double affine Hecke algebra of type $\left(C_{n}^{\vee}, C_{n}\right)$.

The following theorem is essentially due to Macdonald [M5]. 
Theorem 3.2.2. (1) $X^{c_{0}}$ is a central element. (2) Each of the following sets

$$
\begin{gathered}
\left\{X^{\lambda} T(w) Y^{\mu^{\prime}} \mid \lambda \in \Lambda, w \in W\left(R_{0}\right), \mu^{\prime} \in L^{\prime}\right\}, \\
\left\{Y^{\mu^{\prime}} T(w) X^{\lambda} \mid \lambda \in \Lambda, w \in W\left(R_{0}\right), \mu^{\prime} \in L^{\prime}\right\}, \\
\left\{X^{\lambda} T(w) \mid \lambda \in \Lambda, w \in W(\Xi)\right\}, \quad\left\{T(w) X^{\lambda} \mid \lambda \in \Lambda, w \in W(\Xi)\right\}
\end{gathered}
$$

forms a free $\mathcal{A}$-basis of $\mathcal{H}(\Xi)$.

Remark. In the original article [M5], the definition of $\mathcal{H}(\Xi)$ is slightly different: $e$ is the positive integer such that $\left\langle L, L^{\prime}\right\rangle=e^{-1} \mathbb{Z}$, except in type III in which case $e=2$. The central element $q_{0}:=X^{c_{0}}$ is considered as a real number such that $0<q_{0}<1 . \tau_{i}$ and $\tau_{i}^{\natural}$ are also considered as positive real numbers. Assume $q_{0}, \tau_{i}$ and $\tau_{i}^{\natural}$ are algebraically free over $\mathbb{Z}$ and $\mathcal{H}(\Xi)$ is defined as an algebra over $K$ with same generators and relations, where $K$ is a subfield of $\mathbb{R}$ containing $q_{0}$, all $\tau_{i}$ and $\tau_{i}^{\natural}$. The original theorem is the following: each of the four sets which is given by replacing $\Lambda$ with $L$ in the above theorem is a $K$-basis of $\mathcal{H}(\Xi)$. But, in our situation, we can prove our statements by the similar argument. So we omit the proof.

Definition 3.2.3. The small double affine Hecke algebra $\mathcal{H}(\Xi)_{s}$ is the subalgebra of $\mathcal{H}(\Xi)$ which is generated by $T(w)\left(w \in W(\Xi)_{s}\right)$ and $X^{\lambda}\left(\lambda \in \Lambda_{s}\right)$.

We remark that $\mathcal{H}(\Xi)_{s}$ is just equal to $\mathcal{H}(\Xi)$ for $\Xi$ of type III.

Assume $\Xi$ of type I or II. By Lemma 3.1.1 (2), Definition 3.2.1 and Theorem 3.2 .2 , we immediately have the following statement.

Corollary 3.2.4. (1) The similar relations as (D1), (D2) and (D3) in Definition 3.2.1 hold in $\mathcal{H}(\Xi)_{s}$. (2) Each of the following sets

$$
\begin{gathered}
\left\{X^{\lambda} T(w) Y^{\mu^{\prime}} \mid \lambda \in \Lambda_{s}, w \in W\left(R_{0}\right), \mu^{\prime} \in Q\left(R_{0}\right)^{\vee}\right\}, \\
\left\{Y^{\mu^{\prime}} T(w) X^{\lambda} \mid \lambda \in \Lambda_{s}, w \in W\left(R_{0}\right), \mu^{\prime} \in Q\left(R_{0}\right)^{\vee}\right\}, \\
\left\{X^{\lambda} T(w) \mid \lambda \in \Lambda_{s}, w \in W(\Xi)_{s}\right\}, \quad\left\{T(w) X^{\lambda} \mid \lambda \in \Lambda_{s}, w \in W(\Xi)_{s}\right\}
\end{gathered}
$$

forms a free $\mathcal{A}$-basis of $\mathcal{H}(\Xi)_{s}$.

Therefore we have the following.

Corollary 3.2.5. As a set $\mathcal{H}(\Xi) / \mathcal{H}(\Xi)_{s} \cong\left(\Lambda / \Lambda_{s}\right) \times \Omega$. Especially $\mathcal{H}(\Xi)_{s}$ is a subalgebra of $\mathcal{H}(\Xi)$ with a finite index. 


\section{$\S 4$. Summary of Elliptic Root Systems}

4.1. Marked Elliptic root systems. Let $F$ be an $(n+2)$ dimensional real vector space with a positive semi-definite symmetric bilinear form $I: F \times F \rightarrow \mathbb{R}$ with the two-dimensional radical which is denoted by $\operatorname{rad}(I)$. If $\alpha \in F$ satisfies $I(\alpha, \alpha) \neq 0$, we say $\alpha$ is a non isotropic vector. For a non isotropic vector $\alpha \in F$, put $\alpha^{\vee}:=2 \alpha / I(\alpha, \alpha)$ and define a reflection $s_{\alpha}$ by $s_{\alpha}(u):=u-I\left(u, \alpha^{\vee}\right) \alpha$ for $u \in F$.

Definition 4.1.1. $\quad$ A set $R$ of non isotropic vectors in $F$ is called an elliptic root system of rank $n$ if the following conditions are satisfied: (i) $Q(R) \otimes_{\mathbb{Z}} \mathbb{R} \cong F$. (Here $Q(R)$ is the additive subgroup of $F$ generated by $R$.) (ii) $s_{\alpha}(R)=R$ for any $\alpha \in R$. (iii) $I\left(\alpha, \beta^{\vee}\right) \in \mathbb{Z}$ for any $\alpha, \beta \in R$. (iv) $R$ is irreducible. That is, there exists no partition of $R$ into two non-empty subsets $R_{1}$ and $R_{2}$ such that $I(\alpha, \beta)=0$ for all $\alpha \in R_{1}$ and $\beta \in R_{2}$.

Let $W(R)$ be the group generated by all reflections $s_{\alpha}(\alpha \in R)$. We call $W(R)$ the elliptic Weyl group.

A subspace $G$ of $\operatorname{rad}(I)$ of rank 1 defined over $\mathbb{Q}$ is called a marking and the pair $(R, G)$ is called a marked elliptic root system.

We fix a generator $\delta_{1}$ of the rank 1 lattice $G \cap Q(R): G \cap Q(R)=\mathbb{Z} \delta_{1}$. For $\alpha \in R$, set $k_{\alpha}:=\inf \left\{k \in \mathbb{Z}_{>0} \mid \alpha+k \delta_{1} \in R\right\}$ and $\alpha^{*}:=\alpha+k_{\alpha} \delta_{1}$.

Let $\pi_{a}: F \rightarrow F / G$ (resp. $\left.\pi_{f}: F \rightarrow F / \operatorname{rad}(I)\right)$ be the natural projection and set $R_{a}:=\pi_{a}(R)\left(\right.$ resp. $\left.\pi_{f}(R):=R_{f}\right) . R_{a}$ (resp. $\left.R_{0}\right)$ is an affine (resp. finite) root system. In the present paper we assume that $R_{f}$ is reduced, which implies that $R_{a}$ is also reduced.

We fix a subset $\Gamma_{a}=\left\{\alpha_{0}, \cdots, \alpha_{n}\right\}$ of $R$ such that $\pi_{a}\left(\Gamma_{a}\right)$ forms a basis of the affine root system $R_{a}$. Let $\delta_{a}$ be the primitive imaginary root of $R_{a}$. Then $\delta_{a}$ can be written in the following form: $\delta_{a}=\sum_{i=0}^{n} n_{i} \pi_{a}\left(\alpha_{i}\right),\left(n_{i} \in \mathbb{Z}_{>0}\right)$. It is well-known that there always exists an element $\alpha \in \Gamma_{a}$, say $\alpha_{0}$, such that $n_{\alpha_{0}}=1$. Set $\delta_{2}:=\sum_{i=0}^{n} n_{i} \alpha_{i} \in R$ and $\theta:=\sum_{i=1}^{n} n_{i} \alpha_{i}$.

By the construction it is easy to see that $Q(R)$ has a following expression:

$$
Q(R)=\left(\bigoplus_{i=0}^{n} \mathbb{Z} \alpha_{i}\right) \oplus \mathbb{Z} \delta_{1}=\left(\bigoplus_{i=1}^{n} \mathbb{Z} \alpha_{i}\right) \oplus \mathbb{Z} \delta_{1} \oplus \mathbb{Z} \delta_{2} .
$$

For $0 \leq i \leq n$, set $m_{i}:=I_{R}\left(\alpha_{i}, \alpha_{i}\right) n_{i} / 2 k_{\alpha_{i}}$, where $I_{R}$ is a constant multiple of $I$ normalized such that $\inf \left\{I_{R}(\alpha, \alpha) \mid \alpha \in R\right\}$ is equal to 2. Consider the subset $\Gamma_{\max }:=\left\{\alpha_{i} \in \Gamma_{a} \mid m_{i}=m_{\max }\right\}$ of $\Gamma_{a}$, where $m_{\max }:=\max \left\{m_{i} \mid 0 \leq\right.$ $i \leq n\}$. Put $\Gamma_{\max }^{*}:=\left\{\alpha_{i}^{*} \mid \alpha_{i} \in \Gamma_{\max }\right\}$. 
Let $(R, G)$ be a marked elliptic root system belonging to $I$. Then $R^{\vee}:=$ $\left\{\alpha^{\vee} \in F \mid \alpha \in R\right\}$ is also an elliptic root system belonging to $I$. Moreover it is known that the same space $G$ defines a marking for $R^{\vee}$. We call the pair $\left(R^{\vee}, G\right)$ the dual marked elliptic root system of $(R, G)$.

4.2. Elliptic Dynkin diagrams. An elliptic Dynkin diagram $\Gamma(R, G)$ for a marked elliptic root system $(R, G)$ is a finite graph given by the following data:

(1) the vertex set of $\Gamma(R, G)$ is $\Gamma:=\Gamma_{a} \cup \Gamma_{\max }^{*}$.

(2) two vertices $\alpha, \beta \in \Gamma$ are connected according to the following conditions:

\begin{tabular}{|c|c|}
\hline$\bigcirc^{\beta}$ & if $I(\alpha, \beta)=I(\beta, \alpha)=0$, \\
\hline$\bigcirc$ & if $I\left(\alpha, \beta^{\vee}\right)=I\left(\beta, \alpha^{\vee}\right)=-1$ \\
\hline$\alpha \bigcirc \stackrel{t}{\longrightarrow} \bigcirc \beta$ & if $I\left(\alpha, \beta^{\vee}\right)=-\mu$ and $I\left(\beta, \alpha^{\vee}\right)=-1$ for $\mu=2,3$, \\
\hline${ }^{\alpha} \bigcirc \stackrel{\infty}{\bigcirc}{ }^{\beta}$ & if $I\left(\alpha, \beta^{\vee}\right)=I\left(\beta, \alpha^{\vee}\right)=-2$, \\
\hline ニニニニ○ $\beta$ & If $I\left(\alpha, \beta^{\vee}\right)=I\left(\beta, \alpha^{\vee}\right)=2$. \\
\hline
\end{tabular}

Afterwards we use the following conventions:

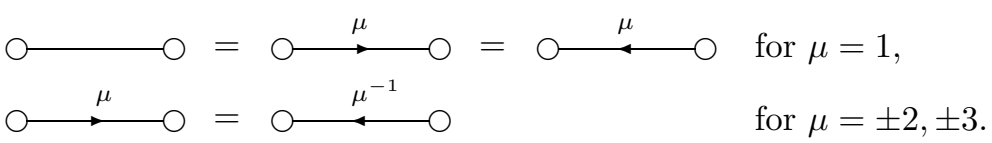

The following theorem is due to K. Saito [S].

Theorem 4.2.1. The isomorphism classes of marked elliptic root systems are completely classified by their elliptic Dynkin diagrams.

In Appendix, we will present a complete list of marked elliptic root systems $(R, G)$ under the assumption that $R_{f}$ is reduced.

By the above classification theorem we have the following lemma.

Lemma 4.2.2. The component $\Gamma(R, G) \backslash\left(\Gamma_{\max } \cup \Gamma_{\max }^{*}\right)=\Gamma_{a} \backslash \Gamma_{\max }$ is a disjoint union of $A$-type diagrams, say $\Gamma\left(A_{l_{1}}\right), \cdots, \Gamma\left(A_{l_{r}}\right)$.

For $\alpha_{i} \in \Gamma_{a}$, we set $\alpha_{i}^{\dagger}:=k_{\alpha_{i}} \alpha_{i}^{\vee}$. It is known that the $\operatorname{set} Q\left((R, G)_{a}\right):=$ $\oplus_{i=0}^{n} \mathbb{Z} \alpha_{i}^{\dagger}$ forms a root lattice of an irreducible reduced affine root system $(R, G)_{a}$ with a basis $\left\{\alpha_{i}^{\dagger}\right\}_{i=0}^{n}$. In order to describe the explicit type of $(R, G)_{a}$, we introduce a grouping of isomorphism classes of marked elliptic root systems due to K. Saito [S]: 
(A) $A_{n}^{(1,1)}(n \geq 1), D_{n}^{(1,1)}(n \geq 4), E_{n}^{(1,1)}(n=6,7,8)$,

(B) $B_{n}^{(1,2)}(n \geq 3), B_{n}^{(2,2)}(n \geq 2), C_{n}^{(1,2)}(n \geq 2), C_{n}^{(2,2)}(n \geq 3), F_{4}^{(1,2)}, F_{4}^{(2,2)}$, $G_{2}^{(1,3)}, G_{2}^{(3,3)}$,

(C) $B_{n}^{(1,1)}(n \geq 3), B_{n}^{(2,1)}(n \geq 2), C_{n}^{(1,1)}(n \geq 2), C_{n}^{(2,1)}(n \geq 3), F_{4}^{(1,1)}, F_{4}^{(2,1)}$, $G_{2}^{(1,1)}, G_{2}^{(3,1)}$,

(D) $A_{1}^{(1,1) *}, B_{n}^{(2,2) *}(n \geq 2), C_{n}^{(1,1) *}(n \geq 2)$.

Theorem 4.2.3. If $(R, G)$ belongs to the group $A, B$ or $C$, we have

$$
Q\left((R, G)_{a}\right) \cong \begin{cases}Q\left(R_{a}\right)=Q\left(R_{a}^{\vee}\right), & \text { if }(R, G) \text { belongs to the group } A, \\ Q\left(R_{a}\right), & \text { if }(R, G) \text { belongs to the group } B, \\ Q\left(R_{a}^{\vee}\right), & \text { if }(R, G) \text { belongs to the group } C .\end{cases}
$$

If $(R, G)$ belongs to the group $D$, we have

$$
Q\left(\left(A_{1}^{(1,1) *}\right)_{a}\right) \cong Q\left(S\left(A_{1}\right)\right), \quad Q\left(\left(B_{n}^{(2,2) *}\right)_{a}\right) \cong Q\left(\left(C_{n}^{(1,1) *}\right)_{a}\right) \cong Q\left(S\left(B C_{n}\right)\right) .
$$

If $(R, G)$ belongs to the group $\mathrm{A}, \mathrm{B}$ or $\mathrm{C}$, there exists the irreducible reduced finite root system $R_{f}^{(0)}$ such that $Q(R, G)_{a}$ is isomorphic to $Q\left(S\left(R_{f}^{(0)}\right)\right)$ or $Q\left(S\left(R_{f}^{(0)}\right)^{\vee}\right)$. But in general, $R_{f}^{(0)}$ is not isomorphic to $R_{f}$.

4.3. Boundary side. Let us introduce the notion of the boundary side due to K. Saito and Takebayashi [ST]. For each pair $\alpha_{i}, \alpha_{j} \in \Gamma_{a}$ which are connected as $\alpha_{i} \circ \stackrel{\mu}{\longrightarrow} \circ \alpha_{j}$ for $\mu=2^{ \pm 1}, 3^{ \pm 1}$, it is known that $k\left(\alpha_{i}, \alpha_{j}\right):=k_{\alpha_{i}} / k_{\alpha_{j}}$ is equal to either 1 or $\mu$.

Definition 4.3.1. $\quad$ In the above setting, $\alpha_{i}$ is called the boundary side (or b-side for short) for the bond $\alpha_{i} \circ \stackrel{\mu}{\longrightarrow} \circ \alpha_{j}$ with $\mu=2^{ \pm 1}, 3^{ \pm 1}$, if $k\left(\alpha_{i}, \alpha_{j}\right)=$ $\inf \{1, \mu\}$.

Remark. For the bond $\alpha_{i} \circ \stackrel{\mu}{\longrightarrow} \circ \alpha_{j}$ for $\mu=2^{ \pm 1}, 3^{ \pm 1}$, either $\alpha_{i}$ or $\alpha_{j}$ is a b-side.

4.4. Hyperbolic extension of elliptic Weyl groups. Let $(R, G)$ be a marked elliptic root system. Consider the pair $(\tilde{F}, \tilde{I})$ of a vector space $\tilde{F}$ over $\mathbb{R}$ and a symmetric bilinear form $\tilde{I}$ on $\tilde{F}$ such that $F$ is a 1-codimensional subspace of $\tilde{F},\left.\tilde{I}\right|_{F}=I$ and $\operatorname{rad}(\tilde{I})=G$. Such $(\tilde{F}, \tilde{I})$ exists uniquely up to isomorphisms. By the definition, we can regard $R$ is a subset of $\tilde{F}$. Let $\tilde{s}_{\alpha} \in O(\tilde{F}, \tilde{I})$ be the reflection with respect to $\alpha \in R$ and $\widetilde{W}(R, G)$ the group 
generated by all reflections $\tilde{s}_{\alpha}(\alpha \in R)$ which is called the hyperbolic extension of $W(R)$.

K. Saito and Takebayashi $[\mathrm{ST}]$ gave a presentation of $\widetilde{W}(R, G)$ by generators attached to the vertices of the elliptic Dynkin diagram $\Gamma(R, G)$ and finitely many relations. In their presentation, in addition to the ordinary Coxeter relations, new relations (so-called elliptic Coxeter relations) appeared. After [ST], Yamada [Y] gave a modification of K. Saito and Takebayashi's presentation for one-codimensional cases. In this article, we generalize Yamada's presentation of $\widetilde{W}(R, G)$ for arbitrary marked elliptic root systems.

The following is K. Saito and Takebayashi's presentation of $\widetilde{W}(R, G)$.

Theorem 4.4.1 ([ST]). $\quad \widetilde{W}(R, G)$ is isomorphic to the group with generators $r_{\alpha}(\alpha \in R)$ subject to the relations explained below.

For any subdiagrams of $\Gamma(R, G)$ isomorphic to the following list, we give relations attach to the diagrams in the following table.

$$
{ }^{\alpha} \bigcirc \quad r_{\alpha}^{2}=1,
$$

$$
{ }^{\alpha} \bigcirc \quad \bigcirc^{\beta} \quad\left(r_{\alpha} r_{\beta}\right)^{2}=1,
$$
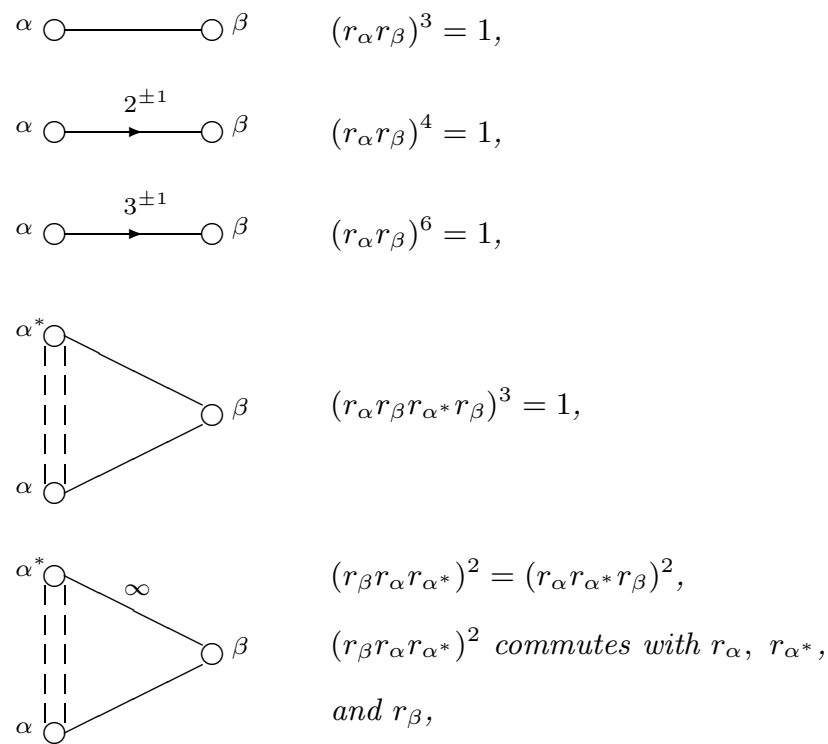
(W2-3)
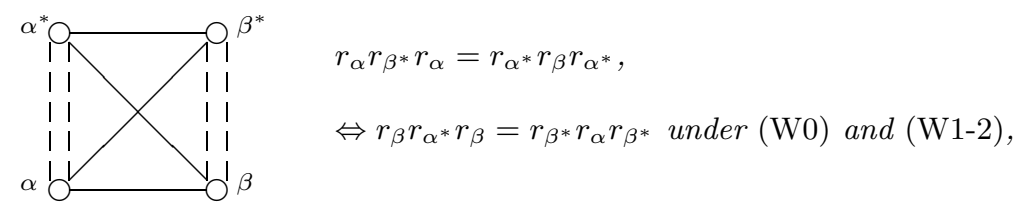

$(\mathrm{W} 2-4)$

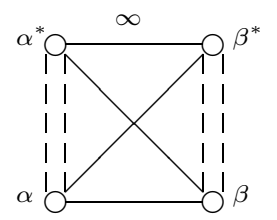

$$
\begin{aligned}
& r_{\alpha} r_{\alpha *} r_{\beta} r_{\beta^{*}}=r_{\alpha^{*}} r_{\beta} r_{\beta^{*}} r_{\alpha} \\
& \quad=r_{\beta} r_{\beta^{*}} r_{\alpha} r_{\alpha^{*}}=r_{\beta^{*}} r_{\alpha} r_{\alpha *} r_{\beta},
\end{aligned}
$$

(W3-1)

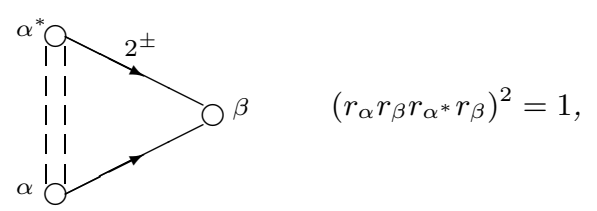

(W3-2)

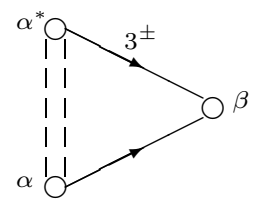

$\left(r_{\alpha} r_{\beta} r_{\alpha *} r_{\beta}\right)^{3}=1$

$\left(r_{\alpha} r_{\beta} r_{\alpha *} r_{\beta} r_{\alpha} r_{\beta}\right)^{2}=1$,

In the next diagram, we assume that $\alpha$ is b-side for the bond $\alpha \circ \stackrel{\mu}{\longrightarrow} \circ \beta$ for $\mu=2^{ \pm 1}, 3^{ \pm 1}$.

(W3-3)

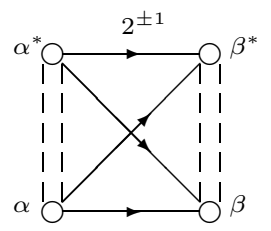

$$
r_{\alpha} r_{\beta^{*}} r_{\alpha}=r_{\alpha *} r_{\beta} r_{\alpha}
$$

In the next diagram, we assume $\mu=1,2^{ \pm 1}, 3^{ \pm 3}$.

(W4)

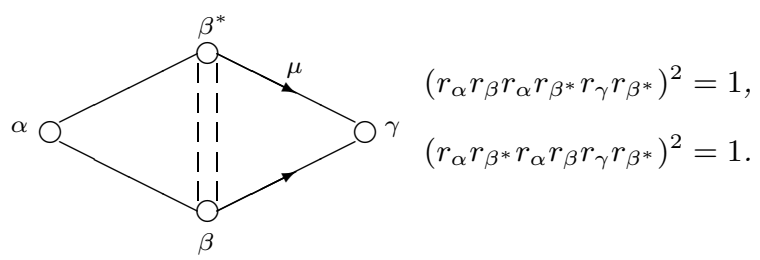

However there are exceptions. In the diagram (W2-4), there are four subdiagrams of type (W2-2). But, we do not assume the relations (W2-2) for these four subdiagrams. We only assume the relations (W2-4).

The relations $(\mathrm{W} 2-1) \sim(\mathrm{W} 4)$ are called elliptic Coxeter relations. 
The following theorem is a generalization of Yamada's presentation $[\mathrm{Y}]$.

Theorem 4.4.2. $\quad \widetilde{W}(R, G)$ has another presentation with generators $r_{\alpha}$ $(\alpha \in R)$ subject to the relations explained below.

For any subdiagrams of $\Gamma(R, G)$ isomorphic to the following list, we give relations attach to the diagrams in the following table.

$$
{ }^{\alpha} \bigcirc \quad r_{\alpha}^{2}=1, \quad \text { (as same as }(\mathrm{W} 0) \text { ), }
$$

$$
{ }^{\alpha} \bigcirc \quad \mathrm{O}^{\beta} \quad r_{\beta}=r_{\beta} r_{\alpha},
$$
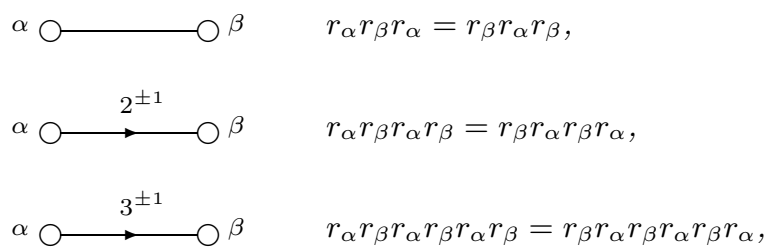

In the following diagrams, we always assume that $\alpha, \beta, \gamma \in \Gamma_{a}$. For $\alpha \in \Gamma_{\max }$, set $x_{\alpha^{\dagger}}=r_{\alpha} r_{\alpha^{*}}$.

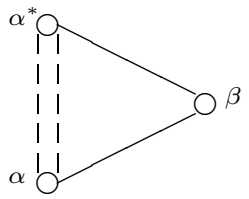

$r_{\beta} x_{\alpha^{\dagger}} r_{\beta} x_{\alpha^{\dagger}}=x_{\alpha^{\dagger}} r_{\beta} x_{\alpha^{\dagger}} r_{\beta}$,

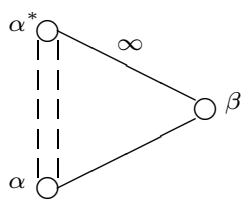

$r_{\beta} x_{\alpha^{\dagger}} r_{\beta} x_{\alpha^{\dagger}}=x_{\alpha^{\dagger}} r_{\beta} x_{\alpha^{\dagger}} r_{\beta}$,

$r_{\beta} x_{\alpha^{\dagger}} r_{\beta} x_{\alpha^{\dagger}}$ commutes with $r_{\alpha}, r_{\alpha^{*}}$, and $r_{\beta}$,

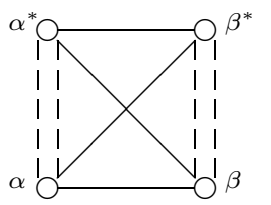

$$
\begin{aligned}
& x_{\beta^{\dagger}} x_{\alpha^{\dagger}}=r_{\beta} x_{\alpha^{\dagger}} r_{\beta}, \\
& \Leftrightarrow x_{\alpha^{\dagger}} x_{\beta^{\dagger}}=r_{\alpha} x_{\beta^{\dagger}} r_{\alpha} \text { under (E0) and (E1-2), }
\end{aligned}
$$


$(\mathrm{E} 2-4)$

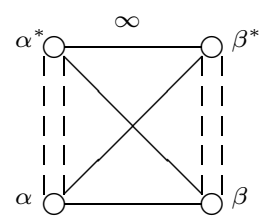

$x_{\beta^{\dagger}} x_{\alpha^{\dagger}}=x_{\alpha^{\dagger}} x_{\beta^{\dagger}}$,

$x_{\alpha^{\dagger}} x_{\beta^{\dagger}}$ commutes with $r_{\alpha}, r_{\alpha^{*}}, r_{\beta^{\dagger}}$ and $r_{\beta^{*}}$.

In the next three diagrams, we assume that $\alpha$ is $b$-side for the bond $\alpha \circ \stackrel{\mu}{\longrightarrow} \circ \beta$ for $\mu=2^{ \pm 1}, 3^{ \pm 1}$.

$(\mathrm{E} 3-1)$

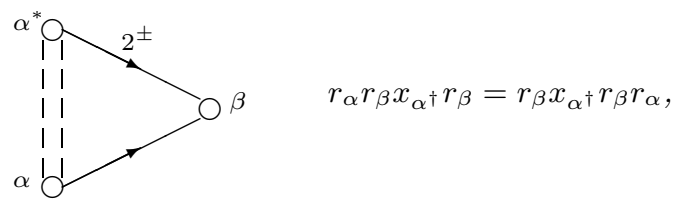

$(\mathrm{E} 3-2)$

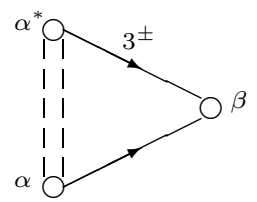

$$
\begin{aligned}
r_{\beta} x_{\alpha^{\dagger}} r_{\beta} x_{\alpha^{\dagger}} & =x_{\alpha^{\dagger}} r_{\beta} x_{\alpha^{\dagger}} r_{\beta} \\
& =r_{\alpha} r_{\beta} x_{\alpha^{\dagger}} r_{\beta} r_{\alpha},
\end{aligned}
$$

(E3-3)

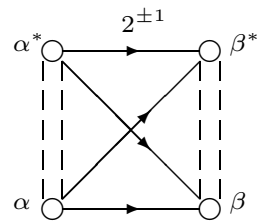

$$
x_{\beta^{\dagger}} x_{\alpha^{\dagger}}=r_{\beta} x_{\alpha^{\dagger}} r_{\beta} .
$$

In the next diagram, we assume $\gamma$ does not belong to $\Gamma_{\max } \cup \Gamma_{\max }^{*}$ and $\mu=$ $1,2^{ \pm 1}, 3^{ \pm 1}$

(E4)

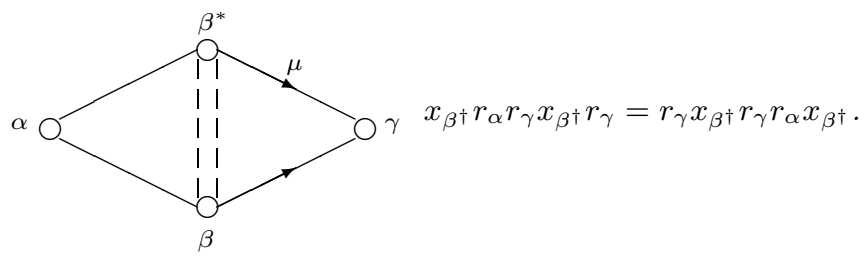

However there are similar exceptions as Theorem 4.4.1. In the diagram (E2-4), there are two subdiagrams of type (E2-2). But, we do not assume the relations (E2-2) for these two subdiagrams. We only assume the relations (E2-4).

It is well-known that the relations (W0), (W1-1), (W1-2), (W1-3), (W1-4) are equivalent to the relations (E0), (E1-1), (E1-2), (E1-3),(E1-4).

The following lemma is due to Yamada [Y]. 
Lemma 4.4.3. Assume the relations (W0), (W1-1), (W1-2), (W1-3), (W1-4) (equivalently, (E0), (E1-1), (E1-2), (E1-3), (E1-4)) hold.

(1) For a subdiagram of the form as (W2-1), we have

$$
\left(r_{\alpha} r_{\beta} r_{\alpha^{*}} r_{\beta}\right)^{3}=1 \quad \Leftrightarrow \quad r_{\beta} x_{\alpha^{\dagger}} r_{\beta} x_{\alpha^{\dagger}}=x_{\alpha^{\dagger}} r_{\beta} x_{\alpha^{\dagger}} r_{\beta} .
$$

(2) For a subdiagram of the form as (W3-1), we have

$$
\left(r_{\alpha} r_{\beta} r_{\alpha^{*}} r_{\beta}\right)^{2}=1 \Leftrightarrow r_{\alpha} r_{\beta} x_{\alpha^{\dagger}} r_{\beta}=r_{\beta} x_{\alpha^{\dagger}} r_{\beta} r_{\alpha} .
$$

(3) For a subdiagram of the form as (W3-2), we have

$$
\begin{aligned}
& \left(r_{\alpha} r_{\beta} r_{\alpha^{*}} r_{\beta}\right)^{3}=1 \quad \text { and } \quad\left(r_{\alpha} r_{\beta} r_{\alpha^{*}} r_{\beta} r_{\alpha} r_{\beta}\right)^{2}=1 \\
& \Leftrightarrow \quad r_{\beta} x_{\alpha^{\dagger}} r_{\beta} x_{\alpha^{\dagger}}=x_{\alpha^{\dagger}} r_{\beta} x_{\alpha^{\dagger}} r_{\beta}=r_{\alpha} r_{\beta} x_{\alpha^{\dagger}} r_{\beta} r_{\alpha} .
\end{aligned}
$$

(4) For a subdiagram of the form as (W4), we have

$$
\begin{aligned}
& \left(r_{\alpha} r_{\beta} r_{\alpha} r_{\beta^{*}} r_{\gamma} r_{\beta^{*}}\right)^{2}=1 \quad \Leftrightarrow \quad x_{\beta^{\dagger}} r_{\alpha} r_{\gamma} x_{\beta^{\dagger}} r_{\gamma}=r_{\gamma} x_{\beta^{\dagger}} r_{\gamma} r_{\alpha} x_{\beta^{\dagger}}, \\
& \left(r_{\alpha} r_{\beta^{*}} r_{\alpha} r_{\beta} r_{\gamma} r_{\beta^{*}}\right)^{2}=1 \quad \Leftrightarrow \quad x_{\beta^{\dagger}} r_{\gamma} r_{\alpha} x_{\beta^{\dagger}} r_{\alpha}=r_{\alpha} x_{\beta^{\dagger}} r_{\alpha} r_{\gamma} x_{\beta^{\dagger}},
\end{aligned}
$$

The following lemma is easily obtained from the definition of $x_{\alpha^{\dagger}}$ and the relation (W0).

Lemma 4.4.4. Under the same assumption as the previous lemma, we have the following:

(1) For a subdiagram of the form as (W2-3),

$$
r_{\alpha} r_{\beta^{*}} r_{\alpha}=r_{\alpha^{*}} r_{\beta} r_{\alpha^{*}} \Leftrightarrow x_{\beta^{\dagger}} x_{\alpha^{\dagger}}=r_{\beta} x_{\alpha} r_{\beta} .
$$

(2) For a subdiagram of the form as (W3-3),

$$
r_{\alpha} r_{\beta^{*}} r_{\alpha}=r_{\alpha^{*}} r_{\beta} r_{\alpha^{*}} \Leftrightarrow x_{\beta^{\dagger}} x_{\alpha^{\dagger}}=r_{\beta} x_{\alpha} r_{\beta} .
$$

(3) For a subdiagram of the form as (W2-4), the following conditions are equivalent.

(i) $r_{\alpha} r_{\alpha^{*}} r_{\beta} r_{\beta^{*}}=r_{\alpha^{*}} r_{\beta} r_{\beta^{*}} r_{\alpha}=r_{\beta} r_{\beta^{*}} r_{\alpha} r_{\alpha^{*}}=r_{\beta^{*}} r_{\alpha} r_{\alpha^{*}} r_{\beta}$.

(ii) $x_{\beta^{\dagger}} x_{\alpha^{\dagger}}=x_{\alpha^{\dagger}} x_{\beta^{\dagger}}$ and $x_{\alpha^{\dagger}} x_{\beta^{\dagger}}$ commutes with $r_{\alpha}, r_{\alpha^{*}}, r_{\beta^{\dagger}}$ and $r_{\beta^{*}}$.

Now we can state the difference of two presentations. In the first presentation, we assume (W2-1) and (W3-1) for all subdiagram of that forms. On the other hand, in the second presentation, we only assume (E2-1) and (E31) for subdiagrams such that $\beta$ belongs to $\Gamma_{a}$ and $\alpha$ is b-side for the bond 
$\alpha \circ \stackrel{\mu}{\longrightarrow} \circ \beta$ for $\mu=2^{ \pm 1}$. Similarly, in the first presentation, we assume (W4) for all subdiagram of that forms. On the other hand, in the second presentation, we only assume (E4) for subdiagrams such that $\gamma$ is not belong to $\Gamma_{\max } \cup \Gamma_{\max }^{*}$ and $\mu=1,2^{ \pm 1}, 3^{ \pm 1}$. These are the difference of two presentations.

We remark that there is no difference between (W3-2) (resp. (W2-2)) and (E3-2) (resp. (E2-2)). By the classification theorem of marked elliptic root systems, in the subdiagram of the following form:

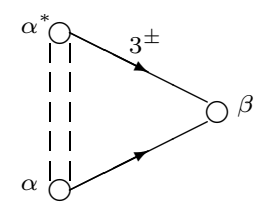

$\beta$ is automatically an element of $\Gamma_{a}$ and $\alpha$ is automatically b-side for the bond $\alpha \circ \stackrel{3^{ \pm 1}}{\longleftarrow} \circ \beta$. Similarly, for the subdiagram of the form as (W2-2) or (E2-2), $\beta$ is automatically an element of $\Gamma_{a}$.

For a proof of Theorem 4.4.2, the remaining problems are the followings: Assume that $r_{\alpha}(\alpha \in R)$ satisfy the relation (E0) $(\mathrm{E} 4)$ (not (W0) $(\mathrm{W} 4)$ ). Then, the problems are;

(a) for all subdiagrams of the form as (W2-1), to prove

$$
r_{\beta} x_{\alpha^{\dagger}} r_{\beta} x_{\alpha^{\dagger}}=x_{\alpha^{\dagger}} r_{\beta} x_{\alpha^{\dagger}} r_{\beta},
$$

(b) for all subdiagrams of the form as (W3-1), to prove

$$
r_{\alpha} r_{\beta} x_{\alpha^{\dagger}} r_{\beta}=r_{\beta} x_{\alpha \dagger} r_{\beta} r_{\alpha},
$$

(c) for all subdiagrams of the form as (W4), to prove

$$
x_{\beta^{\dagger}} r_{\alpha} r_{\gamma} x_{\beta^{\dagger}} r_{\gamma}=r_{\gamma} x_{\beta^{\dagger}} r_{\gamma} r_{\alpha} x_{\beta^{\dagger}} \text { and } \quad x_{\beta^{\dagger}} r_{\gamma} r_{\alpha} x_{\beta^{\dagger}} r_{\alpha}=r_{\alpha} x_{\beta^{\dagger}} r_{\alpha} r_{\gamma} x_{\beta^{\dagger}} \text {. }
$$

Firstly, let us prove (a). If $\beta$ belongs to $\Gamma_{a}$, the formula is nothing but (E2-1). Therefore it is enough to show the following lemma.

Lemma 4.4.5. For the diagram

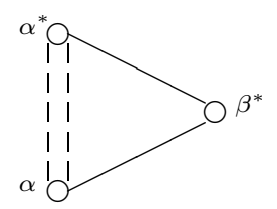


we have $r_{\beta^{*} x_{\alpha^{\dagger}}} r_{\beta^{*}} x_{\alpha^{\dagger}}=x_{\alpha^{\dagger}} r_{\beta^{*}} x_{\alpha^{\dagger}} r_{\beta^{*}}$.

Proof. Such diagram is a subdiagram of

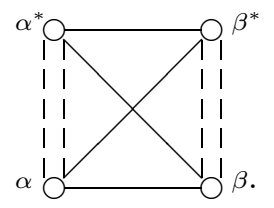

By $(\mathrm{E} 2-3)$ and $x_{\beta^{\dagger}}=r_{\beta} r_{\beta^{*}}$, we have

$$
r_{\beta^{*}}=x_{\alpha^{\dagger}} r_{\beta} x_{\alpha^{\dagger}}^{-1} .
$$

Therefore we have

$$
\begin{aligned}
r_{\beta^{*}} x_{\alpha^{\dagger}} r_{\beta^{*}} x_{\alpha^{\dagger}} & =x_{\alpha^{\dagger}} r_{\beta} x_{\alpha^{\dagger}} r_{\beta} \\
& =x_{\alpha^{\dagger}}\left(r_{\beta} x_{\alpha^{\dagger}} r_{\beta} x_{\alpha^{\dagger}}\right) x_{\alpha^{\dagger}}^{-1} \\
& =x_{\alpha^{\dagger}}^{2} r_{\beta} x_{\alpha^{\dagger}} r_{\beta} x_{\alpha^{\dagger}}^{-1}(\mathrm{by}(\mathrm{E} 2-1)) \\
& =\left(x_{\alpha^{\dagger}} \cdot x_{\alpha^{\dagger}} r_{\beta} x_{\alpha^{\dagger}}^{-1}\right)^{2} \\
& =x_{\alpha^{\dagger}} r_{\beta^{*}} x_{\alpha^{\dagger}} r_{\beta^{*}} .
\end{aligned}
$$

Secondly, let us prove (b). Assume $\alpha$ is b-side for the bond $\alpha \circ \stackrel{2^{ \pm 1}}{\longrightarrow} \beta \beta$ in the diagram of the form as (W3-1). If $\beta \in \Gamma_{a}$, the formula is nothing but (E3-1). For the case of $\beta \notin \Gamma_{a}$, we can prove the formula in the similar way as Lemma 4.4.5. Therefore it is enough to show the following lemma.

Lemma 4.4.6. In the diagram

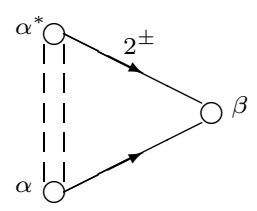

assume that $\beta$ is b-side for the bond $\alpha \stackrel{2^{ \pm 1}}{\longrightarrow} \beta$. Then we have $r_{\alpha} r_{\beta} x_{\alpha^{\dagger}} r_{\beta}=$ $r_{\beta} x_{\alpha \dagger} r_{\beta} r_{\alpha}$

Proof. By the classification of marked elliptic root systems, the above diagram only appeared as a subdiagram of 


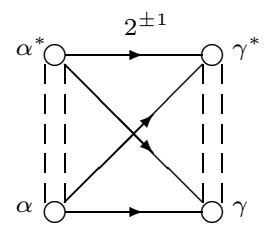

where $\gamma$ is b-side for the bond $\alpha \circ \stackrel{2^{ \pm 1}}{\longrightarrow} \gamma$. We remark that there are two choices of embeddings of diagrams. Namely $\beta=\gamma$ or $\beta=\gamma^{*}$. We give the proof only for the first case. For the second case we prove the formula by the similar way.

By using (E3-3), we have

$$
r_{\alpha^{*}}=r_{\beta} r_{\beta^{*}} r_{\alpha} r_{\beta^{*}} r_{\beta}
$$

Therefore we have

$$
\begin{aligned}
r_{\alpha} r_{\beta} x_{\alpha^{\dagger}} r_{\beta} & =r_{\alpha} r_{\beta} r_{\alpha} r_{\alpha^{*}} r_{\beta} \\
& =r_{\alpha} r_{\beta} r_{\alpha} r_{\beta} r_{\beta^{*}} r_{\alpha} r_{\beta^{*}} r_{\beta}^{2}(\text { by }(4.4 .1)) \\
& =r_{\beta} r_{\alpha} r_{\beta} r_{\alpha} r_{\beta^{*}} r_{\alpha} r_{\beta^{*}}(\text { by (E0) and (E1-3)) } \\
& =r_{\beta} r_{\alpha} r_{\beta} r_{\beta^{*}} r_{\alpha} r_{\beta^{*}} r_{\alpha}(\text { by (E1-3)) } \\
& =r_{\beta} r_{\alpha} r_{\beta} r_{\beta^{*}} r_{\alpha} r_{\beta^{*}} r_{\beta}^{2} r_{\alpha}(\text { by (E0)) } \\
& =r_{\beta} r_{\alpha} r_{\alpha^{*}} r_{\beta} r_{\alpha}(\text { by (4.4.1)) } \\
& =r_{\beta} x_{\alpha^{\dagger}} r_{\beta} r_{\alpha} .
\end{aligned}
$$

We need the next lemma for the proof of (c).

Lemma 4.4.7. In the diagram

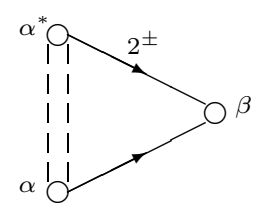

assume that $\alpha$ is b-side for the bond $\alpha \circ \stackrel{2^{ \pm 1}}{\longrightarrow} \beta$. Then we have $r_{\beta} x_{\alpha^{\dagger}} r_{\beta} x_{\alpha^{\dagger}}=$ $x_{\alpha^{\dagger}} r_{\beta} x_{\alpha^{\dagger}} r_{\beta}$.

Proof. It is enough to show that

$$
r_{\beta} r_{\alpha} r_{\alpha^{*}} r_{\beta} r_{\alpha} r_{\alpha^{*}}=r_{\alpha} r_{\alpha^{*}} r_{\beta} r_{\alpha} r_{\alpha^{*}} r_{\beta} .
$$


We have

$$
\begin{aligned}
(4.4 .2) \Leftrightarrow r_{\alpha^{*}} r_{\beta} r_{\alpha} r_{\alpha^{*}} & =r_{\alpha} r_{\beta} r_{\alpha} r_{\alpha^{*}} r_{\beta} r_{\alpha} r_{\alpha^{*}} r_{\beta} \\
& =r_{\beta} r_{\alpha} r_{\alpha^{*}} r_{\beta} r_{\alpha^{*}} r_{\beta}(\mathrm{by}(\mathrm{E} 3-1)) \\
& =r_{\beta} r_{\alpha} r_{\beta} r_{\alpha^{*}} r_{\beta} r_{\alpha^{*}}(\mathrm{by}(\mathrm{E} 1-3)) \\
\Leftrightarrow r_{\alpha} r_{\alpha^{*}} r_{\beta} r_{\alpha} & =r_{\alpha} r_{\beta} r_{\alpha} r_{\beta} r_{\alpha^{*}} r_{\beta} \\
& =r_{\beta} r_{\alpha} r_{\beta} r_{\alpha} r_{\alpha^{*}} r_{\beta}(\mathrm{by}(\mathrm{E} 1-3)) \\
\Leftrightarrow r_{\beta} r_{\alpha} r_{\alpha^{*}} r_{\beta} r_{\alpha} & =r_{\alpha} r_{\beta} r_{\alpha} r_{\alpha^{*}} r_{\beta} .
\end{aligned}
$$

This is nothing but the formula (E3-1).

Finally, let us prove (c). Consider the diagram of the form as (W4):

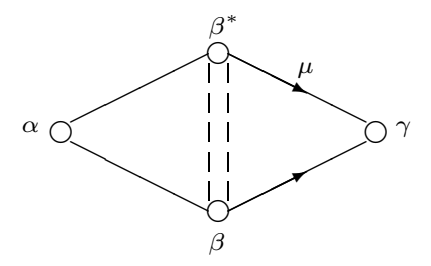

where $\mu=1,2^{ \pm 1}, 3^{ \pm 1}$. It is enough to show the formula for the case that $\gamma \in \Gamma_{\max } \cup \Gamma_{\max }^{*}$. In such case, $\mu=1,2^{ \pm 1}$ by the classification theorem of elliptic Dynkin diagrams.

Lemma 4.4.8. In the above diagram, we assume that $\gamma$ belongs to $\Gamma_{\max }$ $\cup \Gamma_{\text {max }}^{*}$. Then we have $x_{\beta^{\dagger}} r_{\alpha} r_{\gamma} x_{\beta^{\dagger}} r_{\gamma}=r_{\gamma} x_{\beta^{\dagger}} r_{\gamma} r_{\alpha} x_{\beta^{\dagger}}$ and $x_{\beta^{\dagger}} r_{\gamma} r_{\alpha} x_{\beta^{\dagger}} r_{\alpha}=$ $r_{\alpha} x_{\beta^{\dagger}} r_{\alpha} r_{\gamma} x_{\beta^{\dagger}}$.

Proof. In the following, we assume $\gamma \in \Gamma_{\max }$. For the case that $\gamma \in \Gamma_{\max }^{*}$ we can prove the formula by the similar method.

Since $\gamma \in \Gamma_{\max }$, the above diagram is a subdiagram of the following one:

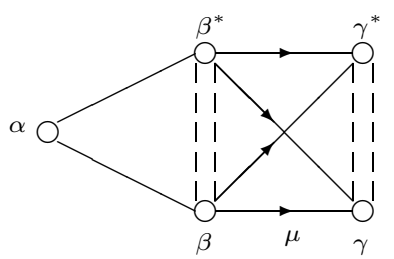

In the following we assume $\mu=2^{ \pm 1}$. For the case that $\mu=1$, we can prove the lemma by the similar methods. 
Let us prove the first formula. By the classification theorem of marked elliptic root systems, $\gamma$ is a b-side for the bond $\beta \stackrel{2^{ \pm 1}}{\longrightarrow} 0 \gamma$.

Let us prove the following formulas:

$$
x_{\beta^{\dagger}} x_{\gamma^{\dagger}}=x_{\gamma^{\dagger}} x_{\beta^{\dagger}},
$$

$$
r_{\gamma} x_{\beta^{\dagger}} r_{\gamma}=x_{\beta^{\dagger}} x_{\gamma^{\dagger}}^{2} .
$$

Since (E3-3) and Lemma 4.4.7, we have (4.4.3). Indeed,

$$
x_{\beta^{\dagger}} x_{\gamma^{\dagger}}=r_{\beta} x_{\gamma^{\dagger}} r_{\beta}=x_{\gamma^{\dagger}} r_{\beta} x_{\gamma^{\dagger}} r_{\beta} x_{\gamma^{\dagger}}^{-1}=x_{\gamma^{\dagger}} x_{\beta^{\dagger}} .
$$

On the other hand,

$$
\begin{aligned}
\text { (4.4.4) } \Leftrightarrow r_{\gamma} x_{\beta^{\dagger}} & =x_{\beta^{\dagger}} x_{\gamma^{\dagger}}^{2} r_{\gamma} \\
& =x_{\gamma^{\dagger}}\left(x_{\beta^{\dagger}} x_{\gamma^{\dagger}}\right) r_{\gamma}(\text { by }(4.4 .3)) \\
& =x_{\gamma^{\dagger}} r_{\beta} x_{\gamma^{\dagger}} r_{\beta} r_{\gamma}(\text { by }(\mathrm{E} 3-3)) \\
& =x_{\gamma^{\dagger}} r_{\gamma} r_{\beta} x_{\gamma^{\dagger}} r_{\beta} \text {. (by (E3-1)) }
\end{aligned}
$$

Since $x_{\gamma^{\dagger}}=r_{\gamma} r_{\gamma^{*}}$, we have

$$
\begin{array}{r}
r_{\gamma} x_{\beta^{\dagger}}=x_{\gamma^{\dagger}} r_{\gamma} r_{\beta} x_{\gamma^{\dagger}} r_{\beta} \Leftrightarrow x_{\beta^{\dagger}}=r_{\gamma^{*}} r_{\gamma} r_{\beta} x_{\gamma^{\dagger}} r_{\beta} \\
=x_{\gamma^{\dagger}}^{-1} r_{\beta} x_{\gamma^{\dagger}} r_{\beta} \\
\Leftrightarrow x_{\beta^{\dagger}} x_{\gamma^{\dagger}}=r_{\beta} x_{\gamma^{\dagger}} r_{\beta} .
\end{array}
$$

This is nothing but (E3-3). Therefore we have (4.4.4).

For the proof of the first formula, it is enough to show the following:

$$
r_{\alpha} r_{\gamma} x_{\beta^{\dagger}} r_{\gamma} x_{\beta^{\dagger}}^{-1}=x_{\beta^{\dagger}}^{-1} r_{\gamma} x_{\beta^{\dagger}} r_{\gamma} r_{\alpha} x_{\beta^{\dagger}} .
$$

By (E1-1), (4.4.3) and (4.4.4) we have

(The left hand side) $=r_{\alpha} x_{\gamma^{\dagger}}^{2}$

$$
\begin{aligned}
& =x_{\gamma^{\dagger}}^{2} r_{\alpha} \\
& =(\text { The right hand side }) .
\end{aligned}
$$


Let us prove the second formula. We have

$$
\begin{aligned}
r_{\gamma} r_{\alpha} x_{\beta^{\dagger}} r_{\alpha} x_{\beta^{\dagger}}^{-1} & =r_{\alpha} r_{\gamma} x_{\beta^{\dagger}} r_{\alpha} x_{\beta^{\dagger}}^{-1}(\text { by }(\mathrm{E} 1-1)) \\
& =r_{\alpha} x_{\beta^{\dagger}} x_{\gamma^{\dagger}}^{2} r_{\gamma} r_{\alpha} x_{\beta^{\dagger}}^{-1}(\mathrm{by}(4.4 .4)) \\
& =r_{\alpha} x_{\beta^{\dagger}} r_{\alpha} x_{\gamma^{\dagger}}^{2} r_{\gamma} x_{\beta^{\dagger}}^{-1} \text { (by (E1-1)) } \\
& =r_{\alpha} x_{\beta^{\dagger}} r_{\alpha} x_{\gamma^{\dagger}}^{2} x_{\gamma^{\dagger}}^{-2} x_{\beta^{\dagger}}^{-1} r_{\gamma}(\text { by (4.4.4)) } \\
& =r_{\alpha} x_{\beta^{\dagger}} r_{\alpha} x_{\beta^{\dagger}}^{-1} r_{\gamma} \\
& =x_{\beta^{\dagger}}^{-1} r_{\alpha} x_{\beta^{\dagger}} r_{\alpha} r_{\gamma} .
\end{aligned}
$$

This is nothing but the second formula. We remark that the last equality follows from (E2-1) if $\alpha \in \Gamma_{a}$ or Lemma 4.4.5 if $\alpha \notin \Gamma_{a}\left(\Leftrightarrow \alpha \in \Gamma_{\max }^{*}\right.$ ).

So the proof of Theorem 4.4.2 is completed.

\section{§5. Elliptic Hecke Algebras}

5.1. Definition of Elliptic Hecke algebras. Let $(R, G)$ be a marked elliptic root system. Consider a Laurent polynomial ring $\mathbb{Z}\left[t_{\alpha}^{ \pm 1}\right]_{\alpha \in \Gamma}$ and let $\mathcal{J}$ be the ideal generated by the elements $t_{\alpha}-t_{\beta}$ where $\alpha$ and $\beta$ are in a same $W(R)$ orbit. Set $\mathbb{A}=\mathbb{Z}\left[t_{\alpha}^{ \pm 1}\right]_{\alpha \in \Gamma} / \mathcal{J}$.

We define the elliptic Hecke algebra $\mathbb{H}=\mathbb{H}(R, G)$ associated with $(R, G)$ as a deformation of the group algebra $\mathbb{Z}[\widetilde{W}(R, G)]$.

Definition 5.1.1. The elliptic Hecke algebra $\mathbb{H}=\mathbb{H}(R, G)$ is an associative $\mathbb{A}$ algebra with generators $g_{\alpha}(\alpha \in \Gamma)$ subject to the relations

$$
{ }^{\alpha} \bigcirc \quad\left(g_{\alpha}-t_{\alpha}\right)\left(g_{\alpha}+t_{\alpha}^{-1}\right)=0
$$

and $(\mathrm{H} 1-1) \sim(\mathrm{H} 4)$ which are obtained from $(\mathrm{E} 1-1) \sim(\mathrm{E} 4)$ by replacing $r_{\alpha}$ with $g_{\alpha}$. Here we set $x_{\alpha^{\dagger}}:=g_{\alpha} g_{\alpha^{*}}$ for $\alpha \in \Gamma_{\max }$.

Remark. (1) In our previous announcement [SS], we assume the other relations

$$
g_{\beta} x_{\alpha^{\dagger}} g_{\beta} x_{\alpha^{\dagger}}=g_{\beta} x_{\alpha^{\dagger}} g_{\beta} x_{\alpha^{\dagger}}
$$

for the subdiagrams of the form as (H3-1) (or (E3-1)). But these relations follows from (H1-3) and (H3-1) by the similar computation as the proof of Lemma 4.4.7. So we omit the above relations form the definition. 
(2) As we already mentioned in the introduction, Takebayashi [T2] defined the elliptic Hecke algebras for marked elliptic root systems except for the group (D) by using the "completed elliptic Dynkin Diagrams". For a given marked elliptic root system except for the group (D), one can show that our algebra is isomorphic to Takebayashi's one. Therefore our definition gives another presentation of Takebayashi's algebra attached by the original elliptic Dynkin diagrams. In this article we omit the proof of the equivalence between our presentation and Takebayashi's one.

(3) Let $\left(R^{\vee}, G\right)$ be the dual marked elliptic root system of $(R, G)$. By the definition, it is easy to see that $\mathbb{H}\left(R^{\vee}, G\right)$ is isomorphic to $\mathbb{H}(R, G)$.

5.2. Lusztig's relations. Recall that, for $\alpha_{i} \in \Gamma_{\max }$, we already defined

$$
x_{\alpha_{i}^{\dagger}}=g_{\alpha_{i}} g_{\alpha_{i}^{*}} .
$$

We remark that it is invertible by the definition.

Let us define $x_{\alpha_{i}^{\dagger}}$ for $\alpha_{i} \in \Gamma_{a} \backslash \Gamma_{\max }$ by the following way.

Definition 5.2.1. Assume $\alpha_{i} \in \Gamma_{a} \backslash \Gamma_{\max }$, then $\alpha_{i}$ belongs to a component $\Gamma\left(A_{l_{k}}\right)=\left\{\beta_{1}, \cdots, \beta_{l_{k}}\right\}$ (cf. Lemma 4.2.2.). Let us consider of the following diagram :

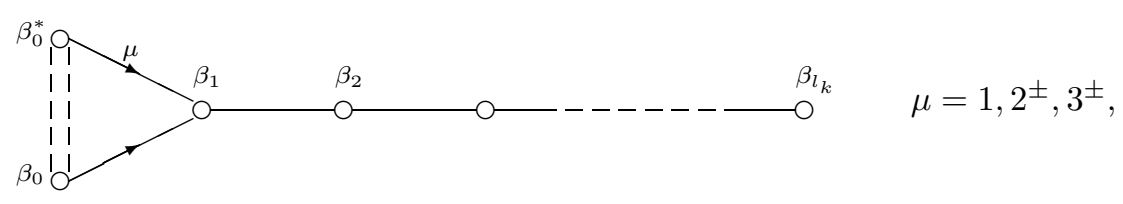

where $\beta_{0}$ is a vertex in $\Gamma_{\max }$ which is connected to $\Gamma\left(A_{l_{k}}\right)$. By using this diagram we define

$$
x_{\beta_{j+1}^{\dagger}}:=g_{\beta_{j+1}} x_{\beta_{j}^{\dagger}} g_{\beta_{j+1}} x_{\beta_{j}^{\dagger}}^{-1} \quad\left(0 \leq j \leq l_{k}-1\right)
$$

inductively. We remark that $x_{\beta_{0}^{\dagger}}$ is already defined by (5.2.1). We also remark that the above $\beta_{0}$ is uniquely determined by the classification theorem of elliptic Dynkin diagrams (Theorem 4.2.1). We say $\beta_{0}$ is the terminal vertex of $\alpha_{i}$ which is denoted by $\alpha_{i}^{t r e m}$.

The following two propositions are key steps in this article.

Proposition 5.2.2. $\quad x_{\alpha_{i}^{\dagger}}\left(\alpha_{i} \in \Gamma_{a}\right)$ are pairwise commutative. In other wards, let $N(R, G)$ be the subalgebra of $\mathbb{H}$ generated by $\left\{x_{\alpha_{i}^{\dagger}}^{ \pm} \mid \alpha_{i} \in \Gamma_{a}\right\}$. Then $N(R, G)$ is a commutative subalgebra of $\mathbb{H}$. 
For $\xi=\xi_{0} \alpha_{0}^{\dagger}+\cdots+\xi_{n} \alpha_{n}^{\dagger} \in Q\left((R, G)_{a}\right)$, set

$$
x_{\xi}:=\left(x_{\alpha_{0}^{\dagger}}\right)^{\xi_{0}} \cdots\left(x_{\alpha_{n}^{\dagger}}\right)^{\xi_{n}} .
$$

By the aid of Proposition 5.2.2, it is well-defined.

Proposition 5.2.3. Lusztig's relations hold in $\mathbb{H}(R, G)$. Namely, we have

$$
g_{\alpha_{i}} x_{\xi}-x_{s_{\alpha_{i}}(\xi)} g_{\alpha_{i}}=\mathbf{b}\left(t_{\alpha_{i}}, t_{\alpha_{i}^{*}} ; x_{\alpha_{i}^{\dagger}}^{-1}\right)\left(x_{\xi}-x_{s_{\alpha_{i}}(\xi)}\right),
$$

for $\alpha_{i} \in \Gamma_{a}, \xi \in Q\left((R, G)_{a}\right)$. Here we set $t_{\alpha_{i}^{*}}:=t_{\alpha_{i}}$ for $\alpha_{i} \in \Gamma_{a} \backslash \Gamma_{\max }$.

Proofs of these propositions will be given in Section 7 .

\subsection{Another presentation of $\mathbb{H}(R, G)$.}

Definition 5.3.1. Let $\hat{\mathbb{H}}(R, G)$ be an associative $\mathbb{A}$ algebra with generators $\hat{g}_{\alpha_{i}}\left(\alpha_{i} \in \Gamma_{a}\right), \hat{x}_{\lambda}\left(\lambda \in Q\left((R, G)_{a}\right)\right)$ subject to the following relations:

$$
\left(\hat{g}_{\alpha_{i}}-t_{\alpha_{i}}\right)\left(\hat{g}_{\alpha_{i}}+t_{\alpha_{i}}^{-1}\right)=0, \quad\left(\alpha_{i} \in \Gamma_{a}\right),
$$

$\left(\mathrm{H}^{\prime} 1-1\right) \quad \hat{g}_{\alpha_{i}} \hat{g}_{\alpha_{j}}=\hat{g}_{\alpha_{j}} \hat{g}_{\alpha_{i}}$

$\alpha_{i} \bigcirc \quad \bigcirc^{\alpha_{j}}$

$$
\hat{g}_{\alpha_{i}} \hat{g}_{\alpha_{j}} \hat{g}_{\alpha_{i}}=\hat{g}_{\alpha_{j}} \hat{g}_{\alpha_{i}} \hat{g}_{\alpha_{j}}
$$

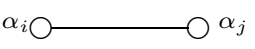

$$
\hat{g}_{\alpha_{i}} \hat{g}_{\alpha_{j}} \hat{g}_{\alpha_{i}} \hat{g}_{\alpha_{j}}=\hat{g}_{\alpha_{j}} \hat{g}_{\alpha_{i}} \hat{g}_{\alpha_{j}} \hat{g}_{\alpha_{i}}
$$

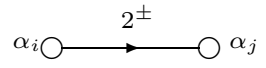

$$
\hat{g}_{\alpha_{i}} \hat{g}_{\alpha_{j}} \hat{g}_{\alpha_{i}} \hat{g}_{\alpha_{j}} \hat{g}_{\alpha_{i}} \hat{g}_{\alpha_{j}}=\hat{g}_{\alpha_{j}} \hat{g}_{\alpha_{i}} \hat{g}_{\alpha_{j}} \hat{g}_{\alpha_{i}} \hat{g}_{\alpha_{j}} \hat{g}_{\alpha_{i}}
$$

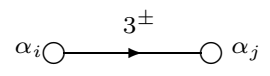

$$
\begin{aligned}
& \hat{x}_{\lambda} \hat{x}_{\mu}=\hat{x}_{\mu} \hat{x}_{\lambda}=\hat{x}_{\lambda+\mu}, \quad \hat{x}_{\lambda} \hat{x}_{-\lambda}=1, \quad\left(\lambda, \mu \in Q\left((R, G)_{a}\right),\right. \\
& \hat{g}_{\alpha_{i}} \hat{x}_{\lambda}-\hat{x}_{s_{\alpha_{i}}(\lambda)} \hat{g}_{\alpha_{i}}=\mathbf{b}\left(t_{\alpha_{i}}, t_{\alpha_{i}^{*}} ; \hat{x}_{\alpha_{i}^{\dagger}}^{-1}\right)\left(\hat{x}_{\lambda}-\hat{x}_{s_{\alpha_{i}}(\lambda)}\right) .
\end{aligned}
$$

The following proposition will be used in the next section and we will prove it in Section 7.

Proposition 5.3.2. $\quad$ There is an isomorphism $\Phi: \mathbb{H}(R, G) \rightarrow \hat{\mathbb{H}}(R, G)$ defined by

$$
g_{\alpha_{i}} \mapsto \hat{g}_{\alpha_{i}}\left(\alpha_{i} \in \Gamma_{a}\right) \quad \text { and } \quad g_{\alpha_{i}^{*}} \mapsto \hat{g}_{\alpha_{i}}^{-1} \hat{x}_{\alpha_{i}^{\dagger}}\left(\alpha_{i}^{*} \in \Gamma_{\max }^{*}\right) .
$$

In other words, the system of the defining relations of $\hat{\mathbb{H}}(R, G)$ gives another presentation of $\mathbb{H}(R, G)$. 
This proposition says that $\mathbb{H}(R, G)$ has another presentation. Namely, $\mathbb{H}(R, G)$ is an algebra with generators $g_{\alpha_{i}}\left(\alpha_{i} \in \Gamma_{a}\right)$ and $x_{\lambda}\left(\lambda \in Q\left((R, G)_{a}\right)\right)$, and relations $\left(\mathrm{H}^{\prime} 0\right) \sim\left(\mathrm{H}^{\prime} 3\right)$ in Definition 5.3.1, by replacing $\hat{g}_{\alpha_{i}}$ and $\hat{x}_{\lambda}$ with $g_{\alpha_{i}}$ and $x_{\lambda}$, respectively.

5.4. PBW-type theorem for $\hat{\mathbb{H}}(R, G)$. For $\mu^{\prime} \in Q\left(R_{f}^{\vee}\right)$, we have introduced $Y^{\mu^{\prime}} \in \mathcal{H}\left(R_{a}\right)$ in Section 2.2. Let us recall the definition. We write $\mu^{\prime} \in Q\left(R_{f}^{\vee}\right)$ as the following form: $\mu^{\prime}=\lambda^{\prime}-\nu^{\prime}$ with $\lambda^{\prime}, \nu^{\prime} \in Q\left(R_{f}^{\vee}\right)^{+}$. We define $Y^{\mu^{\prime}}=$ $T\left(t\left(\lambda^{\prime}\right)\right) T\left(t\left(\nu^{\prime}\right)\right)^{-1}$. By the construction, $T\left(t\left(\lambda^{\prime}\right)\right)$ and $T\left(t\left(\nu^{\prime}\right)\right)$ can be written as

$$
T\left(t\left(\lambda^{\prime}\right)\right)=T_{i_{1}} \cdots T_{i_{N}} \quad \text { and } \quad T\left(t\left(\nu^{\prime}\right)\right)=T_{j_{1}} \cdots T_{j_{M}},
$$

where $w_{i_{1}} \cdots w_{i_{N}}$ and $w_{j_{1}} \cdots w_{i_{M}}$ are reduced expressions in $W\left(R_{a}\right)$ of $t\left(\lambda^{\prime}\right)$ and $t\left(\nu^{\prime}\right)$, respectively.

By the defining relations $\left(\mathrm{H}^{\prime} 0\right) \sim\left(\mathrm{H}^{\prime} 1-4\right)$, we can define a well-defended element $\hat{y}_{\mu^{\prime}}$ in $\hat{\mathbb{H}}(R, G)$ as

$$
\hat{y}_{\mu^{\prime}}:=\hat{g}_{\alpha_{i_{1}}} \cdots \hat{g}_{\alpha_{i_{N}}}\left(\hat{g}_{\alpha_{j_{1}}} \cdots \hat{g}_{\alpha_{i_{M}}}\right)^{-1} \text {. }
$$

By the similar method in [M5], we have PBW-type theorem for $\hat{\mathbb{H}}(R, G)$.

Theorem 5.4.1. Each of the following sets

$$
\begin{aligned}
& \left\{\hat{x}_{\lambda} \hat{g}_{w} \hat{y}_{\mu^{\prime}} \mid \lambda \in Q\left((R, G)_{a}\right), w \in W\left(R_{0}\right), \mu^{\prime} \in Q\left(R_{f}\right)^{\vee}\right\}, \\
& \left\{\hat{y}_{\mu^{\prime}} \hat{g}_{w} \hat{x}_{\lambda} \mid \lambda \in Q\left((R, G)_{a}\right), w \in W\left(R_{0}\right), \mu^{\prime} \in Q\left(R_{f}\right)^{\vee}\right\},
\end{aligned}
$$

$\left\{\hat{x}_{\lambda} \hat{g}_{w} \mid \lambda \in Q\left((R, G)_{a}\right), w \in W\left(R_{a}\right)\right\}, \quad\left\{\hat{g}_{w} \hat{x}_{\lambda} \mid \lambda \in Q\left((R, G)_{a}\right), w \in W\left(R_{a}\right)\right\}$

forms a free $\mathbb{A}$-basis of $\hat{\mathbb{H}}(R, G)$.

Set

$$
y_{\mu^{\prime}}:=\Phi^{-1}\left(\hat{y}_{\mu^{\prime}}\right) \quad\left(\mu^{\prime} \in Q\left(R_{f}\right)^{\vee}\right) .
$$

By Proposition 5.3.2 and Theorem 5.4.1 we have the following corollary.

Corollary 5.4.2. $\quad$ Each of the following sets

$$
\begin{gathered}
\left.\qquad x_{\lambda} g_{w} y_{\mu^{\prime}} \mid \lambda \in Q\left((R, G)_{a}\right), w \in W\left(R_{0}\right), \mu^{\prime} \in Q\left(R_{f}\right)^{\vee}\right\}, \\
\left\{y_{\mu^{\prime}} g_{w} x_{\lambda} \mid \lambda \in Q\left((R, G)_{a}\right), w \in W\left(R_{0}\right), \mu^{\prime} \in Q\left(R_{f}\right)^{\vee}\right\}, \\
\left\{x_{\lambda} g_{w} \mid \lambda \in Q\left((R, G)_{a}\right), w \in W\left(R_{a}\right)\right\}, \quad\left\{g_{w} x_{\lambda} \mid \lambda \in Q\left((R, G)_{a}\right), w \in W\left(R_{a}\right)\right\} \\
\text { forms a free } \mathbb{A} \text {-basis of } \mathbb{H}(R, G) .
\end{gathered}
$$




\section{§6. Elliptic Hecke Algebras and Double Affine Hecke Algebras}

6.1. Triplets associated to elliptic root systems. In this section we assume $(R, G)$ is a marked elliptic root system which belongs to the group A, B or C. Recall that the affine root system $(R, G)_{a}$ is isomorphic to $R_{a}$ or $R_{a}^{\vee}$. Let us consider the following triplet

$$
\Xi(R, G)=\left(R_{f}^{(0)} ; R_{a}, Q\left((R, G)_{a}\right)\right) .
$$

This is a triplet of type I or II in the sense of Section 3.1. In each case, we normalize $I$ so that $I(\theta, \theta)=2$.

For $(R, G)$ of type $A_{1}^{(1,1)}, B_{n}^{(2,2)}$ or $C_{n}^{(1,1)}(n \geq 2)$, we consider another triplet

$$
\Upsilon(R, G)=\left(R_{f}^{(0)} ; R_{a} \cup R_{a}^{\vee}, Q\left((R, G)_{a}\right)\right) .
$$

This is a triplet of type III. In each case, we normalize $I$ so that $I(\theta, \theta)=4$.

6.2. Triplets of type I or II. For a triplet $\Xi(R, G)$, let us consider the corresponding double affine Hecke algebra $\mathcal{H}(\Xi(R, G))$. Since $\Xi(R, G)$ is a triplet of type I or II, $\mathcal{H}(\Xi(R, G))$ is an algebra over $\mathcal{A}=\tilde{\mathcal{A}}_{a}$.

Recall that $\pi_{a}: F \rightarrow F / G$ and $\pi_{a}(R)=R_{a}$. By the definition, the following lemma is easy to verify.

Lemma 6.2.1. Let $\alpha, \beta \in R$. Then the following statements are equivalent. (a) $\alpha$ and $\beta$ are in the same $W(R)$-orbit. (b) $\pi_{a}(\alpha)$ and $\pi_{a}(\beta)$ are in the same $W\left(R_{a}\right)$-orbit.

Let $\mathcal{J}_{a}$ be the ideal of $\mathbb{A}$ generated by the elements $t_{\alpha}-t_{\beta}(\alpha, \beta \in \Gamma)$ where $\pi_{a}(\alpha)=\pi_{a}(\beta)$ and set $\mathbb{A}_{a}:=\mathbb{A} / \mathcal{J}_{a}$. By the construction, $\mathbb{A}_{a}$ is generated by $t_{\alpha_{i}}(0 \leq i \leq n)$. From the above lemma, we easily have;

Corollary 6.2.2. The map $\mathbb{A}_{a} \rightarrow \mathcal{A}_{a}$ defined by $t_{\alpha_{i}} \mapsto \tau_{i}(0 \leq i \leq n)$ is an algebra isomorphism.

From now on, we shall identify these two algebras by the above isomorphism. Let $\mathbb{A} \rightarrow \mathbb{A}_{a}$ and $\mathcal{A}_{a} \rightarrow \tilde{\mathcal{A}}_{a}$ be the natural projections. By taking a composition of these maps, we have a homomorphism $\mathbb{A} \rightarrow \tilde{\mathcal{A}}_{a}$ and we regard $\tilde{\mathcal{A}}_{a}$ as an $\mathbb{A}$-algebra via this homomorphism.

Let us consider an $\tilde{\mathcal{A}}_{a}$-algebra $\tilde{\mathcal{A}}_{a} \otimes_{\mathbb{A}} \mathbb{H}(R, G)$. We remark that the number of unequal parameters of $\tilde{\mathcal{A}}_{a} \otimes_{\mathbb{A}} \mathbb{H}(R, G)$ becomes smaller than one of $\mathbb{H}(R, G)$ in general. 
By the definition of double affine Hecke algebras and Proposition 5.3.2, we have the main theorem of this article.

Theorem 6.2.3. The map $\Theta: \tilde{\mathcal{A}}_{a} \otimes_{\mathbb{A}} \mathbb{H}(R, G) \rightarrow \mathcal{H}(\Xi(R, G))$ defined by

$$
g_{\alpha_{i}} \mapsto T_{i}, \quad x_{\alpha_{i}^{\dagger}} \mapsto X^{\alpha_{i}^{\dagger}} \quad\left(\alpha_{i} \in \Gamma_{a}\right)
$$

is an injective algebra homomorphism and the image just coincides with the small double affine Hecke algebra $\mathcal{H}(\Xi(R, G))_{s}$. Namely, $\tilde{\mathcal{A}}_{a} \otimes_{\mathbb{A}} \mathbb{H}(R, G)$ is isomorphic to $\mathcal{H}(\Xi(R, G))_{s}$.

Proof. By Proposition 5.3.2, $\mathbb{H}(R, G)$ can be regarded as an algebra with generators $g_{\alpha_{i}}\left(\alpha_{i} \in \Gamma_{a}\right)$ and $x_{\lambda}\left(\lambda \in Q\left((R, G)_{a}\right)\right)$, and relations (H'0) $\left(\mathrm{H}^{\prime} 3\right)$ in Definition 5.3.1. Therefore $\Theta$ is well-defined and the image coincides with $\mathcal{H}(\Xi(R, G))_{s}$.

By the construction, we immediately have

$\Theta\left(x_{\lambda} g_{w} y_{\mu^{\prime}}\right)=X^{\lambda} T(w) Y^{\mu^{\prime}} \quad$ for any $\lambda \in Q\left((R, G)_{a}\right), w \in W\left(R_{f}\right), \mu^{\prime} \in Q\left(R_{f}\right)^{\vee}$.

Now the injectivity of $\Theta$ follows from Corollary 3.2.4 and Corollary 5.4.2.

6.3. Triplet of type III. Assume $(R, G)$ is of type $A_{1}^{(1,1)}, B_{n}^{(2,2)}$ or $C_{n}^{(1,1)}$ $(n \geq 2)$. In this case, the coordinate ring $\mathbb{A}$ of the elliptic Hecke algebra $\mathbb{H}(R, G)$ is given as follows;

$$
\mathbb{A}= \begin{cases}\mathbb{Z}\left[t_{0}^{ \pm 1}, t_{0^{*}}^{ \pm 1}, t_{1}^{ \pm 1}, t_{1^{*}}^{ \pm 1}\right], & \text { if }(R, G) \text { is of type } A_{1}^{(1,1)}, \\ \mathbb{Z}\left[t_{0}^{ \pm 1}, t_{0^{*}}^{ \pm 1}, t^{ \pm 1}, t_{n}^{ \pm 1}, t_{n^{*}}^{ \pm 1}\right], & \text { if }(R, G) \text { is of type } B_{n}^{(2,2)} \text { or } C_{n}^{(1,1)}\end{cases}
$$

where $t=t_{i}=t_{i^{*}}(1 \leq i \leq n-1)$.

On the other hand, since $\Upsilon(R, G)$ is a triplet of type III, the corresponding double affine Hecke algebra $\mathcal{H}(\Upsilon(R, G))$ is an algebra over $\mathcal{A}=\mathcal{A}_{a}\left[\left(\tau_{0}^{\natural}\right)^{ \pm 1}\right.$, $\left.\left(\tau_{n}^{\natural}\right)^{ \pm 1}\right]$. Here

$$
\mathcal{A}_{a}= \begin{cases}\mathbb{Z}\left[\tau_{0}^{ \pm 1}, \tau_{1}^{ \pm 1}\right], & \text { if }(R, G) \text { is of type } A_{1}^{(1,1)}, \\ \mathbb{Z}\left[\tau_{0}^{ \pm 1}, \tau^{ \pm 1}, \tau_{n}^{ \pm 1}\right], & \text { if }(R, G) \text { is of type } B_{n}^{(2,2)} \text { or } C_{n}^{(1,1)}\end{cases}
$$

and $\tau=\tau_{i}(1 \leq i \leq n-1)$.

By the above considerations, it immediately follows that;

Lemma 6.3.1. The map defined by

$$
t \mapsto \tau, \quad t_{j} \mapsto \tau_{j}, \quad t_{j^{*}} \mapsto \tau_{j}^{\natural} \quad(j=0, n)
$$

gives an isomorphism $\mathbb{A} \stackrel{\sim}{\rightarrow} \mathcal{A}$. 
By this lemma, we may identify $\mathbb{A}$ with $\mathcal{A}$.

Theorem 6.3.2. The map defined by

$$
g_{\alpha_{i}} \mapsto T_{i}, \quad g_{\alpha_{i}^{*}} \mapsto T_{i}^{-1} X^{\alpha_{i}^{\dagger}} \quad\left(\alpha_{i} \in \Gamma_{a}\right)
$$

gives an isomorphism $\mathbb{H}(R, G) \stackrel{\sim}{\rightarrow} \mathcal{H}(\Upsilon(R, G))$.

Here, we remark that $\Gamma_{a}=\Gamma_{\max }$ since $(R, G)$ is of type $A_{1}^{(1,1)}, B_{n}^{(2,2)}$ or $C_{n}^{(1,1)}(n \geq 2)$.

Proof of Theorem 6.3.2. As same as the proof of Proposition 6.2.3, $\mathbb{H}(R, G)$ can be regarded as an algebra with generators $g_{\alpha_{i}}\left(\alpha_{i} \in \Gamma_{a}\right)$ and $x_{\lambda}\left(\lambda \in Q\left((R, G)_{a}\right)\right)$, and relations (H'0) (H'3) in Definition 5.3.1, by Proposition 5.3.2. By replacing $g_{\alpha_{i}}$ with $T_{i}$ and $x_{\lambda}$ with $X^{\lambda}$, these are nothing but the defining relations of $\mathcal{H}(\Upsilon(R, G))$.

\section{§7. Proofs of the Propositions in Section 5}

7.1. Preliminaries. Let us start from the following lemma which is easily verified by case-by-case checking.

Lemma 7.1.1. Assume $\alpha_{i}, \alpha_{j} \in \Gamma_{a}$ such that $\alpha_{i \circ} \stackrel{\mu^{ \pm 1}}{\longrightarrow} \alpha_{j}(\mu=1,2,3)$. Then the following formula holds:

$$
s_{\alpha_{j}}\left(\alpha_{i}^{\dagger}\right)= \begin{cases}\alpha_{i}^{\dagger}+\mu \alpha_{j}^{\dagger}, & \text { if } \mu \neq 1 \text { and } \alpha_{j} \text { is b-side, } \\ \alpha_{i}^{\dagger}+\alpha_{j}^{\dagger}, & \text { otherwise. }\end{cases}
$$

Lemma 7.1.2. Assume a marked elliptic root system $(R, G)$ is neither of type $A_{1}^{(1,1)}$ nor $A_{1}^{(1,1) *}$. Let $\Gamma\left(A_{l_{k}}\right)=\left\{\beta_{1}, \cdots, \beta_{l_{k}}\right\}$ be a connected component of $\Gamma_{a} \backslash \Gamma_{\max }$ and $\beta_{0} \in \Gamma_{\max }$ the unique vertex which is connected to $\Gamma\left(A_{l_{k}}\right)$ (cf. Definition 5.2.1). Then we have the following formulas:

$$
x_{\beta_{i}^{\dagger}} x_{\beta_{j}^{\dagger}}=x_{\beta_{j}^{\dagger}} x_{\beta_{i}^{\dagger}}\left(0 \leq i, j \leq l_{k}\right) \text {, }
$$

$$
g_{\beta_{i}} x_{\beta_{j}^{\dagger}}-x_{s_{\beta_{i}}\left(\beta_{j}^{\dagger}\right)} g_{\beta_{i}}=\mathbf{b}\left(t_{\beta_{i}}, t_{\beta_{i}^{*}} ; x_{\beta_{i}^{\dagger}}^{-1}\right)\left(x_{\beta_{j}^{\dagger}}-x_{s_{\beta_{i}}\left(\beta_{j}^{\dagger}\right)}\right)\left(0 \leq i, j \leq l_{k}\right)
$$

Proof. For simplicity we denote $g_{i}=g_{\beta_{i}}, x_{i}=x_{\beta_{i}^{\dagger}}\left(i=0, \cdots, l_{k}\right)$ and $\mathbf{b}_{i}=\mathbf{b}\left(t_{\beta_{i}}, t_{\beta_{i}^{*}} ; x_{\beta_{i}^{\dagger}}^{-1}\right)$. By the definition we have $t_{\beta_{i}}=t_{\beta_{i}^{*}}$ for $i=1, \cdots, l_{k}$. Moreover by the classification theorem of marked elliptic root systems (Theorem 4.2.1), it is known that $t_{\beta_{0}}=t_{\beta_{0}^{*}}=t_{\beta_{1}}=\cdots=t_{\beta_{l_{k}}}$. We denote it by $t$. 
Therefore we have $\mathbf{b}_{i}=\left(t-t^{-1}\right) /\left(1-x_{i}^{-1}\right)$. By using Theorem 4.2.1 again, it is also known that $\beta_{0}$ is b-side for the bond $\beta_{0 \circ \longrightarrow} \stackrel{\mu^{ \pm 1}}{\longrightarrow} \beta_{1}$ for $\mu=2,3$. Therefore, by Lemma 7.1.1, we have

$$
s_{\beta_{0}}\left(\beta_{1}^{\dagger}\right)=\mu \beta_{0}^{\dagger}+\beta_{1}^{\dagger} \text { and } s_{\beta_{1}}\left(\beta_{0}^{\dagger}\right)=\beta_{0}^{\dagger}+\beta_{1}^{\dagger} \text { for } \mu=1,2,3 \text {. }
$$

Let us prove (7.1.2). By the definition, the formula $g_{1} x_{0} g_{1} x_{0}=x_{0} g_{1} x_{0} g_{1}$ holds for any $t=1,2^{ \pm 1}, 3^{ \pm 1}$. Recall $x_{1}:=g_{1} x_{0} g_{1} x_{0}^{-1}$. Therefore we have

$$
x_{1} x_{0}=g_{1} x_{0} g_{1}=x_{0} g_{1} x_{0} g_{1} x_{0}^{-1}=x_{0} x_{1}
$$

and

$$
x_{2} x_{0}=\left(g_{2} x_{1} g_{2} x_{1}^{-1}\right) x_{0}=x_{0}\left(g_{2} x_{1} g_{2} x_{1}^{-1}\right)=x_{0} x_{2} .
$$

Next we show $g_{2} x_{1} g_{2} x_{1}=x_{1} g_{2} x_{1} g_{2}$. Indeed, from (H1-1), (H1-2) and (7.1.5), we have

$$
\begin{aligned}
g_{2} x_{1} g_{2} x_{1}-x_{1} g_{2} x_{1} g_{2} & =g_{2} g_{1} x_{0} g_{1} x_{0}^{-1} g_{2} x_{1}-g_{1} x_{0} g_{1} x_{0}^{-1} g_{2} x_{1} g_{2} \\
& =\left(g_{2} g_{1} x_{0} g_{1} g_{2} x_{1}-g_{1} x_{0} g_{1} g_{2} x_{1} g_{2}\right) x_{0}^{-1} \\
& =\left(g_{2} g_{1} x_{0} g_{1} g_{2} g_{1} x_{0} g_{1} x_{0}^{-1}-g_{1} x_{0} g_{1} g_{2} g_{1} x_{0} g_{1} x_{0}^{-1} g_{2}\right) x_{0}^{-1} \\
& =\left(g_{2} g_{1} x_{0} g_{1} g_{2} g_{1} x_{0} g_{1}-g_{1} x_{0} g_{1} g_{2} g_{1} x_{0} g_{1} g_{2}\right) x_{0}^{-2} \\
& =\left(g_{2} g_{1} x_{0} g_{2} g_{1} g_{2} x_{0} g_{1}-g_{1} x_{0} g_{2} g_{1} g_{2} x_{0} g_{1} g_{2}\right) x_{0}^{-2} \\
& =\left(g_{2} g_{1} g_{2} x_{0} g_{1} x_{0} g_{2} g_{1}-g_{1} g_{2} x_{0} g_{1} x_{0} g_{2} g_{1} g_{2}\right) x_{0}^{-2} \\
& =\left(g_{1} g_{2} g_{1} x_{0} g_{1} x_{0} g_{2} g_{1}-g_{1} g_{2} x_{0} g_{1} x_{0} g_{1} g_{2} g_{1}\right) x_{0}^{-2} \\
& =g_{1} g_{2}\left(g_{1} x_{0} g_{1} x_{0}-x_{0} g_{1} x_{0} g_{1}\right) g_{2} g_{1} x_{0}^{-2} \\
& =0 .
\end{aligned}
$$

From this formula and the definition of $x_{2}$ we have

$$
x_{2} x_{1}=g_{2} x_{1} g_{2}=x_{1} g_{2} x_{1} g_{2} x_{1}^{-1}=x_{1} x_{2} \text {. }
$$

By the similar computation, we have (7.1.2).

Let us prove (7.1.3). Firstly we shall prove it for $i=0$ and $j=0$. By (H0) we have

$$
\begin{aligned}
g_{0} x_{0}-x_{0}^{-1} g_{0} & =g_{0}^{2} g_{0^{*}}-g_{0^{*}}^{-1} \\
& =\left\{\left(t-t^{-1}\right) g_{0}+1\right\} g_{0^{*}}-\left\{g_{0^{*}}-\left(t-t^{-1}\right)\right\} \\
& =\left(t-t^{-1}\right)\left(x_{0}+1\right) \\
& =\mathbf{b}_{0}\left(x_{0}-x_{0}^{-1}\right) .
\end{aligned}
$$


This is nothing but the formula.

Secondly let us prove (7.1.3) for $i=0$ and $j=1$. Namely

$$
g_{0} x_{1}-x_{0}^{\mu} x_{1} g_{0}=\mathbf{b}_{0}\left(x_{1}-x_{0}^{\mu} x_{1}\right) .
$$

Assume $\mu=1$. By (H1-2) we have $g_{0^{*}} g_{1} g_{0^{*}}=g_{1} g_{0^{*}} g_{1}$. Then we have $g_{0} x_{1} g_{0}=$ $x_{0} x_{1}$; since $g_{1} x_{0} g_{1}=x_{0} x_{1}$ by $(7.1 .5)$, we have

$$
\begin{aligned}
g_{1} g_{0^{*}} g_{1}=g_{0^{*}} g_{1} g_{0^{*}} & \Rightarrow g_{1} g_{0} g_{1} g_{0^{*}} g_{1} g_{0^{*}}^{-1}=g_{1} g_{0} g_{0^{*}} g_{1} \\
& \Rightarrow g_{0} g_{1} g_{0} g_{0^{*}} g_{1} g_{0^{*}}^{-1}=g_{1} g_{0} g_{0^{*}} g_{1} \\
& \Rightarrow g_{0} x_{1} g_{0}=x_{0} x_{1} .
\end{aligned}
$$

By the above formula and (H0) we have

$$
\begin{aligned}
g_{0} x_{1}-x_{0} x_{1} g_{0} & =g_{0} x_{1}-x_{0} x_{1}\left\{g_{0}^{-1}+\left(t-t^{-1}\right)\right\} \\
& =-\left(t-t^{-1}\right) x_{0} x_{1} \\
& =\mathbf{b}_{0}\left(x_{1}-x_{0} x_{1}\right) .
\end{aligned}
$$

Assume $\mu=2$. By the definition of $x_{1}$, we have $x_{1} x_{0}=g_{1} x_{0} g_{1}$. Therefore, by (H3-1) and (7.1.5), we obtain

$$
g_{0} x_{0} x_{1}=g_{0} g_{1} x_{0} g_{1}=g_{1} x_{0} g_{1} g_{0}=x_{0} x_{1} g_{0} .
$$

By the above formula and (7.1.6) we have

$$
\begin{aligned}
g_{0} x_{1}-x_{0}^{2} x_{1} g_{0} & =\left(g_{0}-x_{0} g_{0} x_{0}\right) x_{1} \\
& =\left\{g_{0}-x_{0}\left(x_{0}^{-1} g_{0}-\mathbf{b}_{0}\left(x_{0}-x_{0}^{-1}\right)\right)\right\} x_{1} \\
& =\mathbf{b}_{0}\left(x_{1}-x_{0}^{2} x_{1}\right) .
\end{aligned}
$$

Assume $\mu=3$. By (H3-2) and the definition of $x_{1}$, we have

$$
x_{0}^{2} x_{1}=g_{0} x_{0} x_{1} g_{0} .
$$

By (7.1.6) and the above formula, we have

$$
\begin{aligned}
g_{0} x_{1}-x_{0}^{3} x_{1} g_{0} & =\left(g_{0}-x_{0}^{2} g_{0}^{-1} x_{0}^{2}\right) x_{1} \\
& =\left\{-\left(t-t^{-1}\right)\left(x_{0}+x_{0}^{2}+x_{0}^{3}\right)\right\} x_{1} \\
& =\mathbf{b}_{0}\left(x_{1}-x_{0}^{3} x_{1}\right) .
\end{aligned}
$$

Thus we have proved (7.1.7). 
We shall prove (7.1.3) for $i=0$ and $j=2$. From (H1-1) and (7.1.7), we have

$$
\begin{aligned}
g_{0} x_{2} & =g_{0} g_{2} x_{1} g_{2} x_{1}^{-1} \\
& =g_{2}\left\{x_{0}^{\mu} x_{1} g_{0}+\mathbf{b}_{0}\left(x_{1}-x_{0}^{\mu} x_{1}\right)\right\} g_{2} x_{1}^{-1} \\
& =g_{2} x_{1} g_{2}\left\{x_{0}^{\mu} g_{0} x_{1}^{-1}+\mathbf{b}_{0}\left(1-x_{0}^{\mu}\right) x_{1}^{-1}\right\} \\
& =g_{2} x_{1} g_{2}\left[x_{0}^{\mu}\left\{x_{0}^{-\mu} x_{1}^{-1} g_{0}+\mathbf{b}_{0}\left(x_{1}^{-1}-x_{0}^{-\mu} x_{1}^{-1}\right)\right\}+\mathbf{b}_{0}\left(1-x_{0}^{\mu}\right) x_{1}^{-1}\right] \\
& =g_{2} x_{1} g_{2} x_{1}^{-1} g_{0} \\
& =x_{2} g_{0} .
\end{aligned}
$$

This is nothing but (7.1.3) for $i=0$ and $j=2$.

By the definition and the formulas we already proved above, we immediately have $g_{0} x_{j}=x_{j} g_{0}(j \geq 3)$. Therefore we have (7.1.3) for $i=0$.

Let us prove (7.1.3) for $i=1$. Since $g_{1} x_{0} g_{1}=x_{0} x_{1}$, we have the formula for $j=0$ by the similar argument of the proof of (7.1.8).

By (H0) we have

$$
\begin{aligned}
g_{1} x_{1}-x_{1}^{-1} g_{1} & =g_{1}^{2} x_{0} g_{1} x_{0}^{-1}-x_{0} g_{1}^{-1} x_{0}^{-1} \\
& =\left\{\left(t-t^{-1}\right) g_{1}+1\right\} x_{0} g_{1} x_{0}^{-1}-x_{0}\left\{g_{1}-\left(t-t^{-1}\right)\right\} x_{0}^{-1} \\
& =\left(t-t^{-1}\right)\left(x_{1}+1\right) \\
& =\mathbf{b}_{1}\left(x_{1}-x_{1}^{-1}\right) .
\end{aligned}
$$

Therefore we have the formula for $j=1$.

Next, we shall prove it for $j=2$. By the similar argument of the proof of (7.1.8), it enough to show that

$$
g_{1} x_{2} g_{1}=x_{1} x_{2}
$$

By (H1-1) and (H1-2) we have

$$
\begin{aligned}
g_{1} x_{2} g_{1} & =g_{1}\left\{g_{2}\left(g_{1} x_{0} g_{1} x_{0}^{-1}\right) g_{2}\left(x_{0} g_{1}^{-1} x_{0}^{-1} g_{1}^{-1}\right)\right\} g_{1} \\
& =g_{2} g_{1} g_{2} x_{0} g_{2}^{-1} g_{1} g_{2} x_{0}^{-1} \\
& =g_{2} x_{1} g_{2} \\
& =x_{1} x_{2} .
\end{aligned}
$$

By the similar argument of the proof of (7.1.11), we have $g_{1} x_{3}=x_{3} g_{1}$. Moreover, by the definition and the formulas we already proved above, we immediately have $g_{1} x_{j}=x_{j} g_{1}(j \geq 4)$. Therefore we have (7.1.3) for $i=1$. 
By the similar computation, we have (7.1.3) for all $i$ and $j$.

Lemma 7.1.3. Let $\Gamma$ is of type $A_{1}^{(1,1) *}$. Then the formulas (7.1.2) and (7.1.3) hold.

Proof. We have $g_{0} x_{1} g_{0} x_{1}=x_{1} g_{0} x_{1} g_{0}$ and $x_{0}=g_{0} x_{1} g_{0} x_{1}^{-1}$ by the definitions. Therefore we have (7.1.2) by the similar way as (7.1.5). Let us prove (7.1.3). In this case, (7.1.3) can be written as

$$
\begin{gathered}
g_{i} x_{i}-x_{i}^{-1} g_{i}=\mathbf{b}_{i}\left(x_{i}-x_{i}^{-1}\right) \quad(i=0,1), \\
g_{0} x_{1}-x_{0} x_{1} g_{0}=\mathbf{b}_{0}\left(x_{1}-x_{0} x_{1}\right), \quad g_{1} x_{0}-x_{0} x_{1}^{4} g_{1}=\mathbf{b}_{1}\left(x_{0}-x_{0} x_{1}^{4}\right)
\end{gathered}
$$

where $\mathbf{b}_{0}=\left(t_{0}-t_{0}^{-1}\right) /\left(1-x_{0}^{-1}\right)$ and $\mathbf{b}_{1}=\left\{\left(t_{1}-t_{1}^{-1}\right)+\left(t_{1^{*}}-t_{1^{*}}{ }^{-1}\right) x_{1}^{-1}\right\} /(1-$ $\left.x_{1}^{-2}\right)$. Since the first and second formulas are proved by the same computations as (7.1.6) and (7.1.8), the remaining problem is the proof of the third formula. By (H2-2), $g_{0} x_{1} g_{0} x_{1}=x_{0} x_{1}^{2}$ is a central element. Therefore we have

$$
g_{1} x_{0}-x_{0} x_{1}^{4} g_{1}=\left(g_{1}-x_{1}^{2} g_{1} x_{1}^{2}\right) x_{0}
$$

By the first formula for $i=1$, we have

$$
\begin{aligned}
x_{1}^{2} g_{1} x_{1}^{2} & =x_{1}^{2}\left(x_{1}^{-1} g_{1}+\mathbf{b}_{1}\left(x_{1}-x_{1}^{-1}\right)\right) x_{1} \\
& =x_{1} g_{1} x_{1}+\mathbf{b}_{1}\left(x_{1}^{4}-x_{1}^{2}\right) \\
& =x_{1}\left(x_{1}^{-1} g_{1}+\mathbf{b}_{1}\left(x_{1}-x_{1}^{-1}\right)\right)+\mathbf{b}_{1}\left(x_{1}^{4}-x_{1}^{2}\right) \\
& =g_{1}+\mathbf{b}_{1}\left(x_{1}^{4}-1\right) .
\end{aligned}
$$

Thus we have the formula.

7.2. Proof of Proposition 5.2.2. The goal of this subsection is to prove the following formula:

$$
x_{\alpha_{i}^{\dagger}} x_{\alpha_{j}^{\dagger}}=x_{\alpha_{j}^{\dagger}} x_{\alpha_{i}^{\dagger}} \text { for } \alpha_{i}, \alpha_{j} \in \Gamma_{a} .
$$

It is enough to prove it in the following three cases: (a) $\alpha_{i}, \alpha_{j} \in \Gamma_{\max }$, (b) $\alpha_{i} \in \Gamma_{\max }$ and $\alpha_{j} \in \Gamma_{a} \backslash \Gamma_{\max },(\mathrm{c}) \alpha_{i}, \alpha_{j} \in \Gamma_{a} \backslash \Gamma_{\max }$.

Case (a); If $\alpha_{i}$ and $\alpha_{j}$ are not connected, the statement is trivial by the definition. Therefore it is enough to show the formula for $i$ and $j$ such that $\alpha_{i \circ \longrightarrow} \stackrel{\mu}{\longrightarrow} \alpha_{j}\left(\mu=1,2^{ \pm}, \infty\right)$. If $\mu=\infty$, the statement is automatically satisfied by the defining relation (H2-4). For the case $\mu=1,2^{ \pm 1}$, we have the formula by the similar computation as (7.1.5). 
Case (b); if $\alpha_{i}=\alpha_{j}^{t e r m}$, the formula is nothing but (7.1.2). Assume $\alpha_{i} \neq$ $\alpha_{j}^{\text {term }}$ and $\alpha_{j} \in \Gamma\left(A_{l_{k}}\right)$. Let us denote $\alpha_{k}=\alpha_{j}^{\text {term }}$. We remark that the formula $x_{i} x_{k}=x_{k} x_{i}$ is already proved in case (a). Since there is no vertex in $\Gamma\left(A_{l_{k}}\right)$ which is connected to $\alpha_{i}$, the statement is trivial by the definition.

Case (c); if $\alpha_{i}$ and $\alpha_{j}$ belong to the same connected component of $\Gamma_{a} \backslash \Gamma_{\max }$, the formula is nothing but (7.1.2). Otherwise, it is trivial by the definition.

So the proof of Proposition 5.2.2 is completed.

\subsection{Proof of Proposition 5.2.3.}

To prove Proposition 5.2.3, it is enough to show the following formula:

$$
g_{\alpha_{i}} x_{\alpha_{j}^{\dagger}}-x_{s_{\alpha_{i}}\left(\alpha_{j}^{\dagger}\right)} g_{\alpha_{i}}=\mathbf{b}\left(t_{\alpha_{i}}, t_{\alpha_{i}^{*}} ; x_{\alpha_{i}^{\dagger}}^{-1}\right)\left(x_{\alpha_{j}^{\dagger}}-x_{s_{\alpha_{i}}\left(\alpha_{j}^{\dagger}\right)}\right)(0 \leq i, j \leq n) .
$$

For simplicity we denote $g_{i}=g_{\alpha_{i}}, x_{i}=x_{\alpha_{i}^{\dagger}}$ and $\mathbf{b}_{i}=\mathbf{b}\left(t_{\alpha_{i}}, t_{\alpha_{i}^{*}} ; x_{\alpha_{i}^{\dagger}}^{-1}\right)$.

By using the method of the proof of (7.1.6), we have the formula for $i=j$ and $\alpha_{i} \in \Gamma_{\max }$.

Assume $i \neq j$. As same as the proof of Proposition 5.2.2, let us consider the following three cases: (a) $\alpha_{i}, \alpha_{j} \in \Gamma_{\max }$, (b) $\alpha_{i} \in \Gamma_{\max }$ and $\alpha_{j} \in \Gamma_{a} \backslash \Gamma_{\max }$, (c) $\alpha_{i}, \alpha_{j} \in \Gamma_{a} \backslash \Gamma_{\max }$.

Case (a); If $\alpha_{i}$ is not connected to $\alpha_{j}$ in the elliptic Dynkin diagram, the statement is clear by (H1-1). Assume $\alpha_{i}$ is connected to $\alpha_{j}$. By the classification theorem of elliptic Dynkin diagrams, there are the following three cases;

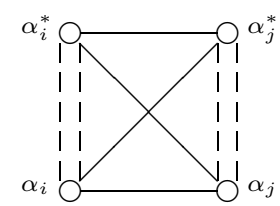

(ii)

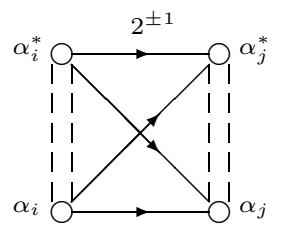

(iii)

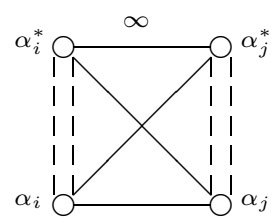

For the case (i), (7.3.1) can be written as

$$
g_{i} x_{j}-x_{i} x_{j} g_{i}=\mathbf{b}_{i}\left(x_{j}-x_{i} x_{j}\right), \quad g_{j} x_{i}-x_{i} x_{j} g_{j}=\mathbf{b}_{j}\left(x_{i}-x_{i} x_{j}\right) .
$$

Since $g_{i} x_{j} g_{i}=g_{j} x_{i} g_{j}=x_{i} x_{j}$, we have the statement by the similar computation of the proof of (7.1.8).

For the case (ii), we may assume $\alpha_{i}$ is b-side. In such case, (7.3.1) can be written as

$$
g_{i} x_{j}-x_{i}^{2} x_{j} g_{i}=\mathbf{b}_{i}\left(x_{j}-x_{i}^{2} x_{j}\right), \quad g_{j} x_{i}-x_{i} x_{j} g_{j}=\mathbf{b}_{j}\left(x_{i}-x_{i} x_{j}\right) .
$$


Since $g_{j} x_{i} g_{j}=x_{i} x_{j}$, we have the second formula by the same way as the case (i).

Let us prove the first formula. We remark that there is the following subdiagram.

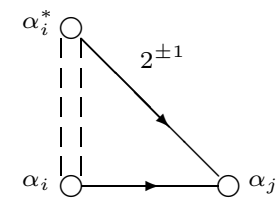

Then the first formula is proved by the similar method as the proof of (7.1.9).

For the case (iii), (7.3.1) can be written as

$$
g_{i} x_{j}-x_{i}^{2} x_{j} g_{i}=\mathbf{b}_{i}\left(x_{j}-x_{i}^{2} x_{j}\right), \quad g_{j} x_{i}-x_{i} x_{j}^{2} g_{j}=\mathbf{b}_{j}\left(x_{i}-x_{i} x_{j}^{2}\right) .
$$

Since $x_{i} x_{j}$ is a central element, we have $g_{i} x_{i} x_{j}=x_{i} x_{j} g_{i}$ and $g_{j} x_{i} x_{j}=x_{i} x_{j} g_{j}$. Therefore we have the statement as same as the proof of (7.1.9).

Case (b); If $\alpha_{i}=\alpha_{j}^{\text {term }}$ we already proved the formula in Lemma 7.1.2 and Lemma 7.1.3. Assume $\alpha_{i} \neq \alpha_{j}^{\text {term }}$. Then $\alpha_{i}$ and $\alpha_{j}$ live in the following positions:

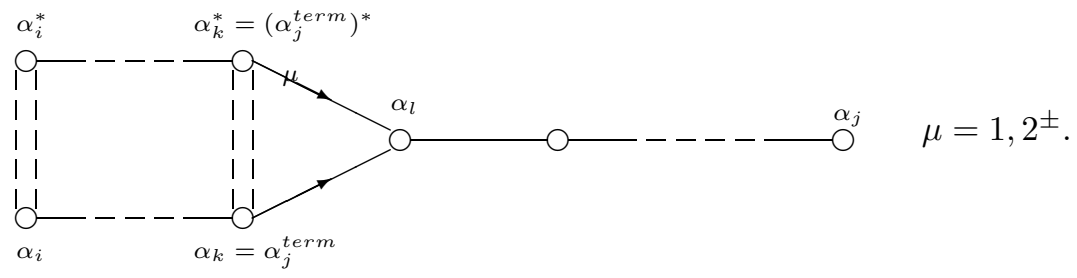

Since $\alpha_{i}$ and $\alpha_{j}$ are not connected, (7.3.1) can be written as

$$
g_{i} x_{j}=x_{j} g_{i} \quad \text { and } \quad g_{j} x_{i}=x_{i} g_{j} .
$$

The second formula is clear by the definition. If $\alpha_{i}$ is not connected to $\alpha_{k}=$ $\alpha_{j}^{\text {term }}$, the first formula is also trivial. From now on we assume $\alpha_{i}$ is connected to $\alpha_{k}$ and, in the following, we will give the proof of the first one for the case that $\alpha_{j}=\alpha_{l}$. In the other cases, it is proved by the similar computation.

We remark that, in such case, both $\alpha_{i}$ and $\alpha_{k}$ belong to $\Gamma_{\max }$ and are connected by a bond such that $\mu=1$. Therefore we already proved the following formula in the case (a): $g_{i} x_{k}-x_{i} x_{k} g_{i}=\mathbf{b}_{i}\left(x_{k}-x_{i} x_{k}\right)$. Then we have the first formula of (7.3.2) by the similar method of the proof of (7.1.11). 
Case (c); If both $\alpha_{i}$ and $\alpha_{j}$ are in the same connected component of $\Gamma_{a} \backslash$ $\Gamma_{\max }$, (7.3.1) is already proved in Lemma 7.1.2. Otherwise, $\alpha_{i}$ is not connected to $\alpha_{j}$. Therefore (7.3.1) can be written as $g_{i} x_{j}=x_{j} g_{i}$ and $g_{j} x_{i}=x_{i} g_{j}$. Firstly assume $\alpha_{i}^{\text {term }}=\alpha_{j}^{\text {term }}$. Then $\alpha_{i}$ and $\alpha_{j}$ live in the following positions:

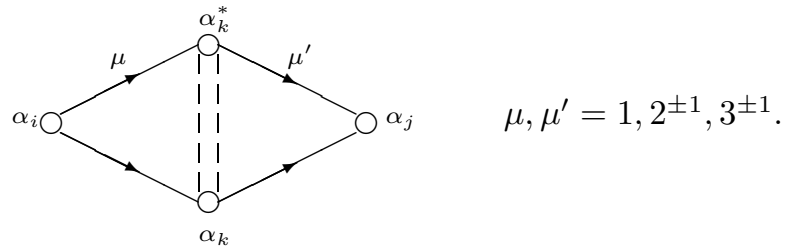

Here we denote $\alpha_{k}=\alpha_{i}^{\text {term }}=\alpha_{j}^{\text {term }}$. By (H2-1), (H3-1) and (H3-2), we have $g_{i} x_{k} g_{i} x_{k}=x_{k} g_{i} x_{k} g_{i}$ for any $\mu$ and $\mu^{\prime}$. On the other hand we have

$$
x_{k} g_{j} g_{i} x_{k} g_{i}=g_{i} x_{k} g_{i} g_{j} x_{k} \quad \Leftrightarrow \quad g_{j} g_{i} x_{k} g_{i} x_{k}^{-1}=x_{k}^{-1} g_{i} x_{k} g_{i} g_{j} .
$$

by (H4). Therefore we have

$$
g_{j} x_{i}=g_{j} g_{i} x_{k} g_{i} x_{k}^{-1}=x_{k}^{-1} g_{i} x_{k} g_{i} g_{j}=g_{i} x_{k} g_{i} x_{k}^{-1} g_{j}=x_{i} g_{j} .
$$

The formula $g_{i} x_{j}=x_{j} g_{i}$ is also proved by the similar method.

Secondly assume $\alpha_{i}^{\text {term }} \neq \alpha_{j}^{\text {term }}$. In such case, by (H1-1), $g_{j}$ commutes with $x_{\left(\alpha_{i}^{t e r m}\right)^{\dagger}}$. Moreover $\alpha_{j}$ is not connected to the connected component of $\Gamma_{a} \backslash \Gamma_{\max }$ which contains $\alpha_{i}$. Therefore we have $g_{j} x_{i}=x_{i} g_{j}$. The formula $g_{i} x_{j}=x_{j} g_{i}$ can be proved by the similar way.

So the proof of Proposition 5.2.3 is completed.

7.4. Proof of Proposition 5.3.2 I (Hecke relations and Coxeter relations). In this and next subsection we will show the well-definedness of the map $\Phi$. For this purpose, in this subsection, we will show that Hecke relations and usual Coxeter relations hold in $\hat{\mathbb{H}}(R, G)$.

For simplicity we denote $t_{i}=t_{\alpha_{i}}, \hat{g}_{i}=\hat{g}_{\alpha_{i}}, \hat{x}_{i}=\hat{x}_{\alpha_{i}^{\dagger}}$ for $\alpha_{i} \in \Gamma_{a}$ and $t_{j^{*}}=t_{\alpha_{j}^{*}}, \hat{g}_{j^{*}}=\hat{g}_{\alpha_{j}}^{-1} \hat{x}_{\alpha_{j}^{\dagger}}$ for $\alpha_{j}^{*} \in \Gamma_{\max }^{*}$.

Lemma 7.4.1. For any $\alpha_{j}^{*} \in \Gamma_{\text {max }}^{*}$, we have $\left(\hat{g}_{j^{*}}-t_{j^{*}}\right)\left(\hat{g}_{j^{*}}-t_{j^{*}}^{-1}\right)=0$.

Proof. By (H'3) we have

$$
\hat{g}_{j} \hat{x}_{j}-\hat{x}_{j}^{-1} \hat{g}_{j}=\hat{\mathbf{b}}_{j}\left(\hat{x}_{j}-\hat{x}_{j}^{-1}\right) .
$$

Here we denote $\hat{\mathbf{b}}_{j}=\mathbf{b}\left(t_{j}, t_{j^{*}} ; \hat{x}_{j}^{-1}\right)=\left\{\left(t_{j}-t_{j}^{-1}\right)+\left(t_{j^{*}}-t_{j^{*}}^{-1}\right) \hat{x}_{j}^{-1}\right\} /\left(1-\hat{x}_{j}^{-2}\right)$. Therefore the right hand side is equal to $\left(t_{j}-t_{j}^{-1}\right) \hat{x}_{j}+\left(t_{j^{*}}-t_{j^{*}}^{-1}\right)$. By $\left(\mathrm{H}^{\prime} 0\right)$ 
and the above equality we have

$$
\begin{aligned}
\hat{g}_{j}^{-1} \hat{x}_{j} & =\left\{\hat{g}_{j}-\left(t_{j}-t_{j}^{-1}\right)\right\} \hat{x}_{j} \\
& =\hat{x}_{j}^{-1} \hat{g}_{j}+\left(t_{j^{*}}-t_{j^{*}}^{-1}\right) .
\end{aligned}
$$

Therefore we have

$$
\begin{aligned}
\left(\hat{g}_{j}^{-1} \hat{x}_{j}\right)^{2} & =\left\{\hat{x}_{j}^{-1} \hat{g}_{j}+\left(t_{j^{*}}-t_{j^{*}}^{-1}\right)\right\} \hat{g}_{j}^{-1} \hat{x}_{j} \\
& =1+\left(t_{j^{*}}-t_{j^{*}}^{-1}\right) \hat{g}_{j}^{-1} \hat{x}_{j} .
\end{aligned}
$$

Hence we have the statement.

The following statement is trivial by the definition.

Lemma 7.4.2. Assume $\alpha_{j^{*}}$ is not connected to $\alpha_{i}$ (resp. $\left.\alpha_{i^{*}}\right)$ in $\Gamma(R$, $G)$, then $\hat{g}_{j^{*}} \hat{g}_{i}=\hat{g}_{i} \hat{g}_{j^{*}}\left(\right.$ resp. $\left.\hat{g}_{j^{*}} \hat{g}_{i^{*}}=\hat{g}_{i^{*}} \hat{g}_{j^{*}}\right)$.

From now on we assume $\alpha_{i} \in \Gamma_{a}, \alpha_{j} \in \Gamma_{\max }$ such that $\alpha_{i \circ} \stackrel{\mu^{ \pm 1}}{\longrightarrow} \circ \alpha_{j}$ for $\mu=$ $1,2,3$. Moreover we assume $\alpha_{j}$ is the b-side for $\mu=2,3$. By the classification theorem of elliptic Dynkin diagram and the definition of the coordinate ring $\mathbb{A}$ we have the following:

(a) If $\mu=1$ then $t_{j}=t_{j^{*}}$ in $\mathbb{A}$.

(b) Assume $\mu=2$. If the vertex $\alpha_{i^{*}}$ appears in $\Gamma$ then $t_{i}=t_{i^{*}}$ and $t_{j} \neq t_{j^{*}}$ in

A. On the other hand, if $\alpha_{i^{*}}$ does not appear in $\Gamma$ then $t_{j}=t_{j^{*}}$.

(c) If $\mu=3$ then $\alpha_{i^{*}}$ does not appear in $\Gamma$ and $t_{j}=t_{j^{*}}$.

Lemma 7.4.3. Let us denote $\sharp=i$ or $j$. Under the above assumption we have the following. (1) If $s_{\alpha_{\sharp}}\left(a \alpha_{i}^{\dagger}+b \alpha_{j}^{\dagger}\right)=a \alpha_{i}^{\dagger}+b \alpha_{j}^{\dagger}$, then $\hat{g}_{\sharp} \hat{x}_{i}^{a} \hat{x}_{j}^{b}=\hat{x}_{i}^{a} \hat{x}_{j}^{b} \hat{g}_{\sharp}$. (2) If $t_{\sharp}=t_{\sharp^{*}}$ in $\mathbb{A}$ and $s_{\alpha_{\sharp}}\left(a \alpha_{i}^{\dagger}+b \alpha_{j}^{\dagger}\right)=a \alpha_{i}^{\dagger}+b \alpha_{j}^{\dagger}+\alpha_{\sharp}^{\dagger}$, then $\hat{g}_{\sharp} \hat{x}_{i}^{a} \hat{x}_{j}^{b} \hat{g}_{\sharp}=\hat{x}_{i}^{a} \hat{x}_{j}^{b} \hat{x}_{\sharp}$.

Proof. The first statement is trivial by the definition. Let us show the second one. By (H'3) we have

$$
\hat{g}_{\sharp} \hat{x}_{i}^{a} \hat{x}_{j}^{b}-\hat{x}_{i}^{a} \hat{x}_{j}^{b} \hat{x}_{\sharp} \hat{g}_{\sharp}=\hat{\mathbf{b}}_{\sharp}\left(\hat{x}_{i}^{a} \hat{x}_{j}^{b}-\hat{x}_{i}^{a} \hat{x}_{j}^{b} \hat{x}_{\sharp}\right) .
$$

Since $t_{\sharp}=t_{\sharp^{*}}$, we have $\hat{\mathbf{b}}_{\sharp}=\left(t_{\sharp}-t_{\sharp}^{-1}\right) /\left(1-\hat{x}_{\sharp}^{-1}\right)$. Therefore the right hand side is equal to $-\left(t_{\sharp}-t_{\sharp}^{-1}\right) \hat{x}_{i}^{a} \hat{x}_{j}^{b} \hat{x}_{\sharp}$. By the Hecke relation for $\hat{g}_{\sharp}$, we have the statement.

Lemma 7.4.4. Let $\alpha_{i} \in \Gamma_{a}, \alpha_{j} \in \Gamma_{\max }$ be as same as in Lemma 7.4.3. Moreover assume $t_{j}=t_{j^{*}}$ in $\mathbb{A}$. Then the corresponding Coxeter relation for $\hat{g}_{i}$ and $\hat{g}_{j^{*}}$ holds. 
Proof. Firstly we assume $\mu=1$. In this case, the corresponding Coxeter relation is

$$
\hat{g}_{i} \hat{g}_{j^{*}} \hat{g}_{i}=\hat{g}_{j^{*}} \hat{g}_{i} \hat{g}_{j^{*}}
$$

and we claim that the condition $t_{j}=t_{j^{*}}$ is automatically satisfied. By the definition of $\hat{g}_{j^{*}}$ and the Coxeter relation for $\hat{g}_{i}$ and $\hat{g}_{j}$, one can easily see that (7.4.1) is equivalent to the following formula (as same as in the proof of (7.1.7)):

$$
\hat{g}_{j} \hat{x}_{i} \hat{g}_{j}=\hat{x}_{i} \hat{x}_{j}
$$

But this formula holds from Lemma $7.4 .3(2)$ because $s_{\alpha_{j}}\left(\alpha_{i}^{\dagger}\right)=\alpha_{i}^{\dagger}+\alpha_{j}^{\dagger}$.

Secondly assume $\mu=2$. Then the corresponding Coxeter relation is

$$
\hat{g}_{i} \hat{g}_{j^{*}} \hat{g}_{i} \hat{g}_{j^{*}}=\hat{g}_{j *} \hat{g}_{i} \hat{g}_{j *} \hat{g}_{i}
$$

By Lemma 7.4.3 we have

$$
\hat{g}_{i} \hat{x}_{j} \hat{g}_{i}=\hat{x}_{i} \hat{x}_{j}, \quad \hat{g}_{j} \hat{x}_{i} \hat{x}_{j}=\hat{x}_{i} \hat{x}_{j} \hat{g}_{j} .
$$

By using the above formulas and the Coxeter relation for $\hat{g}_{i}$ and $\hat{g}_{j}$, it is easy to see that (7.4.3) is equivalent to the following formula:

$$
\hat{g}_{i} \hat{x}_{i} \hat{x}_{j}^{2}=\hat{x}_{i} \hat{x}_{j}^{2} \hat{g}_{i}
$$

Indeed, since $\hat{g}_{j^{*}}=\hat{g}_{j}^{-1} \hat{x}_{j}$

$$
\begin{aligned}
(7.4 .3) & \Leftrightarrow \hat{g}_{i} \hat{g}_{j}^{-1} \hat{x}_{j} \hat{g}_{i} \hat{g}_{j}^{-1} \hat{x}_{j}=\hat{g}_{j}^{-1} \hat{x}_{j} \hat{g}_{i} \hat{g}_{j}^{-1} \hat{x}_{j} \hat{g}_{i} \\
& \Leftrightarrow \hat{g}_{i} \hat{g}_{j}^{-1} \hat{g}_{i}^{-1}\left(\hat{g}_{i} \hat{x}_{j} \hat{g}_{i}\right) \hat{g}_{j}^{-1} \hat{x}_{j}=\hat{g}_{j}^{-1} \hat{g}_{i}^{-1}\left(\hat{g}_{i} \hat{x}_{j} \hat{g}_{i}\right) \hat{g}_{j}^{-1} \hat{x}_{j} \hat{g}_{i} \\
& \Leftrightarrow \hat{g}_{j} \hat{g}_{i} \hat{g}_{j}^{-1} \hat{g}_{i}^{-1} \hat{x}_{i} \hat{x}_{j} \hat{g}_{j}^{-1} \hat{x}_{j}=\hat{g}_{i}^{-1} \hat{x}_{i} \hat{x}_{j} \hat{g}_{j}^{-1} \hat{x}_{j} \hat{g}_{i} \\
& \Leftrightarrow \hat{g}_{i}^{-1} \hat{g}_{j}^{-1} \hat{g}_{i} \hat{g}_{j} \hat{x}_{i} \hat{x}_{j} \hat{g}_{j}^{-1} \hat{x}_{j}=\hat{g}_{i}^{-1} \hat{x}_{i} \hat{x}_{j} \hat{g}_{j}^{-1} \hat{x}_{j} \hat{g}_{i} \\
& \Leftrightarrow \hat{g}_{j}^{-1} \hat{g}_{i} \hat{x}_{i} \hat{x}_{j}^{2}=\hat{g}_{j}^{-1} \hat{x}_{i} \hat{x}_{j}^{2} \hat{g}_{i} \\
& \Leftrightarrow \hat{g}_{i} \hat{x}_{i} \hat{x}_{j}^{2}=\hat{x}_{i} \hat{x}_{j}^{2} \hat{g}_{i} .
\end{aligned}
$$

This is nothing but (7.4.5). Since $s_{\alpha_{i}}\left(\alpha_{i}^{\dagger}+2 \alpha_{j}^{\dagger}\right)=\alpha_{i}^{\dagger}+2 \alpha_{j}^{\dagger}$, (7.4.5) holds by Lemma $7.4 .3(1)$.

Finally assume $\mu=3$. Then the corresponding Coxeter relation is

$$
\hat{g}_{i} \hat{g}_{j^{*}} \hat{g}_{i} \hat{g}_{j^{*}} \hat{g}_{i} \hat{g}_{j^{*}}=\hat{g}_{j^{*}} \hat{g}_{i} \hat{g}_{i} \hat{g}_{j^{*}} \hat{g}_{j^{*}} \hat{g}_{i}
$$

and the condition $t_{j}=t_{j^{*}}$ is automatically satisfied. By Lemma 7.4.3 we have

$$
\hat{g}_{i} \hat{x}_{j} \hat{g}_{i}=\hat{x}_{i} \hat{x}_{j}, \quad \hat{g}_{i} \hat{x}_{i} \hat{x}_{j}^{2}=\hat{x}_{i} \hat{x}_{j}^{2} \hat{g}_{i}, \quad \hat{g}_{j} \hat{x}_{i} \hat{x}_{j}=\hat{x}_{i} \hat{x}_{j}^{2} \hat{g}_{j}^{-1} .
$$


Therefore we have

$$
\begin{aligned}
\hat{g}_{i} \hat{g}_{j^{*}} \hat{g}_{i} \hat{g}_{j^{*}} \hat{g}_{i} \hat{g}_{j^{*}} & =\hat{g}_{i} \hat{g}_{j}^{-1} \hat{x}_{j} \hat{g}_{i} \hat{g}_{j}^{-1} \hat{x}_{j} \hat{g}_{i} \hat{g}_{j}^{-1} \hat{x}_{j} \\
& =\hat{g}_{i} \hat{g}_{j}^{-1} \hat{g}_{i}^{-1} \hat{x}_{i} \hat{x}_{j} \hat{g}_{j}^{-1} \hat{x}_{j} \hat{g}_{i} \hat{g}_{j}^{-1} \hat{x}_{j} \\
& =\hat{g}_{i} \hat{g}_{j}^{-1} \hat{g}_{i}^{-1} \hat{x}_{j}^{-1} \hat{g}_{j} \hat{x}_{i} \hat{x}_{j}^{2} \hat{g}_{i} \hat{g}_{j}^{-1} \hat{x}_{j} \\
& =\hat{g}_{i} \hat{g}_{j}^{-1} \hat{x}_{i}^{-1} \hat{x}_{j}^{-1} \hat{g}_{i} \hat{g}_{j} \hat{g}_{i} \hat{x}_{i} \hat{x}_{j} \hat{g}_{j}^{-1} \hat{x}_{j} \\
& =\hat{g}_{i} \hat{x}_{i}^{-1} \hat{x}_{j}^{-2} \hat{g}_{j} \hat{g}_{i} \hat{g}_{j} \hat{g}_{i} \hat{g}_{j} \hat{x}_{i} \hat{x}_{j}^{2} \\
& =\hat{x}_{i}^{-1} \hat{x}_{j}^{-2} \hat{g}_{i} \hat{g}_{j} \hat{g}_{i} \hat{g}_{j} \hat{g}_{i} \hat{g}_{j} \hat{x}_{i} \hat{x}_{j}^{2}
\end{aligned}
$$

On the other hand, by a similar computation, we have

$$
\hat{g}_{j^{*}} \hat{g}_{i} \hat{g}_{j^{*}} \hat{g}_{i} \hat{g}_{j^{*}} \hat{g}_{i}=\hat{x}_{i}^{-1} \hat{x}_{j}^{-2} \hat{g}_{j} \hat{g}_{i} \hat{g}_{j} \hat{g}_{i} \hat{g}_{j} \hat{g}_{i} \hat{x}_{i} \hat{x}_{j}^{2}
$$

By the Coxeter relation for $\hat{g}_{i}$ and $\hat{g}_{j}$ we have the statement.

Lemma 7.4.5. Let $\alpha_{i}, \alpha_{j} \in \Gamma_{\max }$ be as same as in Lemma 7.4 .3 with $\mu=1$. Then we have $\hat{g}_{i^{*}} \hat{g}_{j^{*}} \hat{g}_{i^{*}}=\hat{g}_{j^{*}} \hat{g}_{i^{*}} \hat{g}_{j^{*}}$.

Proof. By Lemma 7.4.3 we have

$$
\begin{aligned}
\hat{g}_{i^{*}} \hat{g}_{j^{*}} \hat{g}_{i^{*}} & =\hat{g}_{i}^{-1} \hat{x}_{i} \hat{g}_{j}^{-1} \hat{x}_{j} \hat{g}_{i}^{-1} \hat{x}_{i} \\
& =\hat{g}_{i}^{-1} \hat{x}_{j}^{-1} \hat{g}_{j} \hat{x}_{i} \hat{x}_{j} \hat{g}_{i}^{-1} \hat{x}_{i} \\
& =\hat{x}_{i}^{-1} \hat{x}_{j}^{-1} \hat{g}_{i} \hat{g}_{j} \hat{g}_{i} \hat{x}_{i} \hat{x}_{j} .
\end{aligned}
$$

By a similar computation we have

$$
\hat{g}_{j^{*}} \hat{g}_{i^{*}} \hat{g}_{j^{*}}=\hat{x}_{i}^{-1} \hat{x}_{j}^{-1} \hat{g}_{j} \hat{g}_{i} \hat{g}_{j} \hat{x}_{i} \hat{x}_{j}
$$

Therefore we have the statement by the Coxeter relation for $\hat{g}_{i}$ and $\hat{g}_{j}$.

Assume $\alpha_{i}, \alpha_{j} \in \Gamma_{\max }$ such that $\alpha_{i} \stackrel{2^{ \pm 1}}{\longrightarrow} \alpha_{j}$ and $\alpha_{j}$ is the b-side. Namely we consider the following situation:

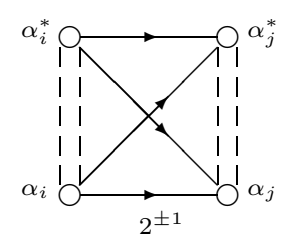

In this case we recall that $t_{i}=t_{i^{*}}$ and $t_{i} \neq t_{i^{*}}$ in $\mathbb{A}$. 
The remaining problems are: (i) The Coxeter relation for $\hat{g}_{i}$ and $\hat{g}_{j^{*}}$, (ii) That for $\hat{g}_{i^{*}}$ and $\hat{g}_{j}$, (iii) That for $\hat{g}_{i^{*}}$ and $\hat{g}_{j^{*}}$.

We have (i) by the same computation as the proof of (7.4.3).

Let us prove (ii). By Lemma 7.4.3 we have

$$
\hat{g}_{i} \hat{x}_{j} \hat{g}_{i}=\hat{x}_{i} \hat{x}_{j}, \quad \text { and } \quad \hat{g}_{j} \hat{x}_{i} \hat{x}_{j}=\hat{x}_{i} \hat{x}_{j} \hat{g}_{j} .
$$

Therefore we have

$$
\begin{aligned}
\hat{g}_{j} \hat{g}_{i^{*}} \hat{g}_{j} \hat{g}_{i^{*}} & =\hat{g}_{j} \hat{g}_{i}^{-1} \hat{x}_{i} \hat{g}_{j} \hat{g}_{i}^{-1} \hat{x}_{i} \\
& =\hat{g}_{j} \hat{g}_{i}^{-1} \hat{x}_{j}^{-1} \hat{g}_{j} \hat{x}_{i} \hat{x}_{j} \hat{g}_{i}^{-1} \hat{x}_{i} \\
& =\hat{g}_{j} \hat{x}_{i}^{-1} \hat{x}_{j}^{-1} \hat{g}_{i} \hat{g}_{j} \hat{g}_{i} \hat{x}_{i} \hat{x}_{j} \\
& =\hat{x}_{i}^{-1} \hat{x}_{j}^{-1} \hat{g}_{j} \hat{g}_{i} \hat{g}_{j} \hat{g}_{i} \hat{x}_{i} \hat{x}_{j} .
\end{aligned}
$$

On the other hand we have

$$
\hat{g}_{i^{*}} \hat{g}_{j} \hat{g}_{i^{*}} \hat{g}_{j}=\hat{x}_{i}^{-1} \hat{x}_{j}^{-1} \hat{g}_{i} \hat{g}_{j} \hat{g}_{i} \hat{g}_{j} \hat{x}_{i} \hat{x}_{j}
$$

by the similar way. Therefore we have $\hat{g}_{j} \hat{g}_{i^{*}} \hat{g}_{j} \hat{g}_{i^{*}}=\hat{g}_{i^{*}} \hat{g}_{j} \hat{g}_{i^{*}} \hat{g}_{j}$ by the Coxeter relation for $\hat{g}_{i}$ and $\hat{g}_{j}$.

Finally, we shall prove $\hat{g}_{j^{*}} \hat{g}_{i^{*}} \hat{g}_{j^{*}} \hat{g}_{i^{*}}=\hat{g}_{i^{*}} \hat{g}_{j^{*}} \hat{g}_{i^{*}} \hat{g}_{j^{*}}$. By the similar computation as the proof of (ii), we have

$$
\begin{aligned}
& \hat{g}_{j^{*}} \hat{g}_{i^{*}} \hat{g}_{j^{*}} \hat{g}_{i^{*}}=\hat{x}_{i}^{-1} \hat{x}_{j}^{-1} \hat{g}_{j}^{-1} \hat{g}_{i}^{-1} \hat{g}_{j}^{-1} \hat{g}_{i} \hat{x}_{i}^{2} \hat{x}_{j}^{3} \quad \text { and } \\
& \hat{g}_{i^{*}} \hat{g}_{j^{*}} \hat{g}_{i^{*}} \hat{g}_{j^{*}}=\hat{x}_{i}^{-1} \hat{x}_{j}^{-1} \hat{g}_{i} \hat{g}_{j}^{-1} \hat{g}_{i}^{-1} \hat{g}_{j}^{-1} \hat{x}_{i}^{2} \hat{x}_{j}^{3}
\end{aligned}
$$

By the Coxeter relation for $\hat{g}_{i}$ and $\hat{g}_{j}$ we have the statement.

Thus, we have proved all Hecke and Coxeter relations.

7.5. Proof of Proposition 5.3.2 II (elliptic Coxeter relations). Firstly let us prove elliptic Coxeter relations $(\mathrm{H} 2-1) \sim(\mathrm{H} 4)$ except for $(\mathrm{H} 2-2)$ and (H2-4).

(H2-1) Since $s_{\beta}\left(\beta^{\dagger}\right)=\alpha^{\dagger}+\beta^{\dagger}$ and $t_{\beta}=t_{\beta^{*}}$, we have $\hat{g}_{\beta} \hat{x}_{\alpha^{\dagger}} \hat{g}_{\beta}=\hat{x}_{\alpha^{\dagger}} \hat{x}_{\beta^{\dagger}}$ by Lemma 7.4.3. Therefore the formula comes from (H'2).

(H2-3) The formulas are already proved in Lemma 7.4.3 (2).

(H3-1) The formula comes from Lemma 7.4.3 (1). Indeed, it is equivalent to $\hat{g}_{\alpha} \hat{x}_{\alpha^{\dagger}} \hat{x}_{\beta^{\dagger}}=\hat{x}_{\alpha^{\dagger}} \hat{x}_{\beta^{\dagger}} \hat{g}_{\alpha}$. Since $\alpha$ is b-side, $s_{\alpha}\left(\alpha^{\dagger}+\beta^{\dagger}\right)=\left(-\alpha^{\dagger}\right)+2 \alpha^{\dagger}+$ $\beta^{\dagger}=\alpha^{\dagger}+\beta^{\dagger}$. Therefore we have the formula. 
(H3-2) The first equality is proved by the same argument as (H2-1). The second one is equivalent to $\hat{x}_{\alpha^{\dagger}}^{2} \hat{x}_{\beta^{\dagger}}=\hat{g}_{\alpha} \hat{x}_{\alpha^{\dagger}} \hat{x}_{\beta^{\dagger}} \hat{g}_{\alpha}$. As we already mentioned in the last subsection, $t_{\alpha}=t_{\alpha^{*}}$. Moreover $s_{\alpha}\left(\alpha^{\dagger}+\beta^{\dagger}\right)=2 \alpha^{\dagger}+\beta^{\dagger}$. Therefore we have the second equality by Lemma $7.4 .3(2)$.

(H3-3) Since $\alpha$ is b-side, $t_{\beta}=t_{\beta^{*}}$ and $s_{\beta}\left(\alpha^{\dagger}\right)=\alpha^{\dagger}+\beta^{\dagger}$. Thus we have the formula form Lemma $7.4 .3(2)$.

(H4) Since $\alpha$ is not belong to $\Gamma_{\max }$, for the bond $\alpha_{\circ} \stackrel{\mu^{ \pm 1}}{\longrightarrow} \beta, \mu=1$, or $\mu=$ 2,3 and $\beta$ is b-side. Therefore we have $\hat{g}_{\alpha} \hat{x}_{\beta^{\dagger}} \hat{g}_{\alpha}=\hat{x}_{\alpha^{\dagger}} \hat{x}_{\beta^{\dagger}}$. Therefore the formula is reduced to $\hat{x}_{\beta^{\dagger}} \hat{g}_{\gamma} \hat{x}_{\alpha^{\dagger}} \hat{x}_{\beta^{\dagger}}=\hat{x}_{\beta^{\dagger}} \hat{x}_{\alpha^{\dagger}} \hat{g}_{\gamma} \hat{x}_{\beta^{\dagger}}$. Since $\alpha$ and $\gamma$ are not connected, $\hat{g}_{\gamma} \hat{x}_{\alpha^{\dagger}}=\hat{x}_{\alpha^{\dagger}} \hat{g}_{\gamma}$. Thus we have a statement.

Let us prove (H2-2). This diagram only appears in of type $A_{1}^{(1,1) *}$. ( $\alpha=\alpha_{1}$ and $\beta=\alpha_{0}$.) In this case, $s_{0}\left(\alpha_{1}^{\dagger}\right)=\alpha_{0}^{\dagger}+\alpha_{1}^{\dagger}$ and $t_{0}=t_{0^{*}}$. Then we have the first formula by the similar argument as (H2-1).

We remark that $\hat{g}_{0} \hat{x}_{1} \hat{g}_{0} \hat{x}_{1}=\hat{x}_{0} \hat{x}_{1}^{2}$. Since $s_{\sharp}\left(\alpha_{0}^{\dagger}+2 \alpha_{1}^{\dagger}\right)=\alpha_{0}^{\dagger}+2 \alpha_{1}^{\dagger}(\sharp=0,1)$, we have $\hat{g}_{\sharp} \hat{x}_{0} \hat{x}_{1}^{2}=\hat{x}_{0} \hat{x}_{1}^{2} \hat{g}_{\sharp}$ by the similar computations as Lemma 7.4.3 (1). Since $\hat{g}_{1^{*}}=\hat{g}_{1}^{-1} \hat{x}_{1}, \hat{g}_{1^{*}}$ also commutes with $\hat{x}_{0} \hat{x}_{1}^{2}$.

Finally we shall prove (H2-4). This diagram only appears in of type $A_{1}^{(1,1)}$. ( $\alpha=\alpha_{0}$ and $\beta=\alpha_{1}$.) The first equality is nothing but one of the defining relations. Since $s_{\sharp}\left(\alpha_{0}^{\dagger}+\alpha_{1}^{\dagger}\right)=\alpha_{0}^{\dagger}+\alpha_{1}^{\dagger}(\sharp=0,1)$, we can show that $\hat{x}_{0} \hat{x}_{1}$ is a central element by the similar argument as the proof of (H2-2).

So we have all elliptic Coxeter relations.

7.6. Proof of Proposition 5.3.2 III. Let us prove Proposition 5.3.2. From 7.3 and 7.4 , we already know the map $\Phi: \mathbb{H}(R, G) \rightarrow \hat{\mathbb{H}}(R, G)$ defined by

$$
g_{\alpha_{i}} \mapsto \hat{g}_{\alpha_{i}}\left(\alpha_{i} \in \Gamma_{a}\right) \quad \text { and } \quad g_{\alpha_{i}^{*}} \mapsto \hat{g}_{\alpha_{i}}^{-1} \hat{x}_{\alpha_{i}^{\dagger}}\left(\alpha_{i}^{*} \in \Gamma_{\max }^{*}\right)
$$

is a well-defined algebra homomorphism.

Let us consider $\Psi: \hat{\mathbb{H}}(R, G) \rightarrow \mathbb{H}(R, G)$ defined by

$$
\hat{g}_{\alpha_{i}} \mapsto g_{\alpha_{i}} \quad \text { and } \quad \hat{x}_{\alpha_{i}^{\dagger}} \mapsto x_{\alpha_{i}^{\dagger}}
$$

for $\alpha_{i} \in \Gamma_{a}$. By Proposition 5.2.2 and 5.2.3, the map $\Psi$ is also a well-defined algebra homomorphism. Since

$$
\Psi \circ \Phi\left(g_{\alpha_{i}}\right)=\Psi\left(\hat{g}_{\alpha_{i}}\right)=g_{\alpha_{i}} \quad\left(\alpha_{i} \in \Gamma_{a}\right)
$$




$$
\Psi \circ \Phi\left(g_{\alpha_{i}^{*}}\right)=\Psi\left(\hat{g}_{\alpha_{i}}^{-1} \hat{x}_{\alpha_{i}^{\dagger}}\right)=g_{\alpha_{i}^{*}} \quad\left(\alpha_{i}^{*} \in \Gamma_{\max }^{*}\right),
$$

$\Psi \circ \Phi$ is the identity map of $\mathbb{H}(R, G)$.

Let us prove $\Phi \circ \Psi$ is the identity map of $\hat{\mathbb{H}}(R, G)$. The following formulas are obvious:

$$
\begin{gathered}
\Phi \circ \Psi\left(\hat{g}_{\alpha_{i}}\right)=\hat{g}_{\alpha_{i}} \quad\left(\alpha_{i} \in \Gamma_{a}\right), \\
\Phi \circ \Psi\left(\hat{x}_{\alpha_{j}^{\dagger}}\right)=\hat{x}_{\alpha_{j}^{\dagger}} \quad\left(\alpha_{j} \in \Gamma_{\max }\right) .
\end{gathered}
$$

For $\alpha_{j} \in \Gamma_{a} \backslash \Gamma_{\max }$, recall that $\beta_{0}:=\alpha_{j}^{\text {term }} \in \Gamma_{\max }$ and $\beta_{l}:=\alpha_{j}$ live in the following positions in the elliptic Dynkin diagram:

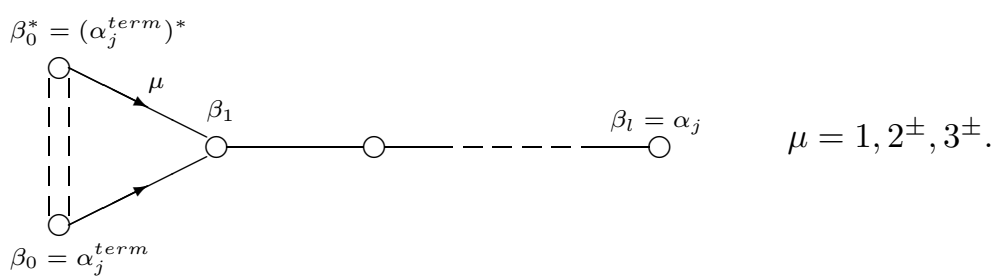

Since all Hecke, Coxeter and elliptic Coxeter relations hold in $\hat{\mathbb{H}}(R, G)$, we have

$$
\hat{x}_{\beta_{m+1}^{\dagger}}=\hat{g}_{\beta_{m+1}} \hat{x}_{\beta_{m}^{\dagger}} \hat{g}_{\beta_{m+1}} \hat{x}_{\beta_{m}^{\dagger}}^{-1} \quad(0 \leq m \leq l-1) .
$$

Since $\beta_{0}=\alpha_{j}^{\text {term }} \in \Gamma_{\max }$, we have $\Phi \circ \Psi\left(\hat{x}_{\beta_{0}^{\dagger}}\right)=\hat{x}_{\beta_{0}^{\dagger}}$. By induction on $m$, we have

$$
\begin{aligned}
\Phi \circ \Psi\left(\hat{x}_{\beta_{m+1}^{\dagger}}\right) & =\Phi \circ \Psi\left(\hat{g}_{\beta_{m+1}} \hat{x}_{\beta_{m}^{\dagger}} \hat{g}_{\beta_{m+1}} \hat{x}_{\beta_{m}^{\dagger}}^{-1}\right) \\
& =\hat{g}_{\beta_{m+1}} \hat{x}_{\beta_{m}^{\dagger}} \hat{g}_{\beta_{m+1}} \hat{x}_{\beta_{m}^{\dagger}}^{-1} \\
& =\hat{x}_{\beta_{m+1}^{\dagger}} .
\end{aligned}
$$

Therefore $\Phi \circ \Psi\left(\hat{x}_{\alpha_{j}^{\dagger}}\right)=\hat{x}_{\alpha_{j}^{\dagger}}$ for all $\alpha_{j} \in \Gamma_{a}$. Namely, $\Phi \circ \Psi$ is the identity map of $\hat{H}(R, G)$ and the proof of Proposition 5.3.2 is completed.

\section{Appendix A. A List of Reduced Affine Root Systems}

We will present a complete list of reduced affine root systems. In this article, we use Macdonald's notations for the types of reduced affine root systems. (See [M5].) In this list, $\varepsilon_{1}, \varepsilon_{2}, \cdots$ is a sequence of orthonormal vectors in a real vector space.

For each root system, we shall exhibit the Dynkin diagram and

(a) the name of that type, in Kac's notation;

(b) roots of that type; 
(c) a basis.

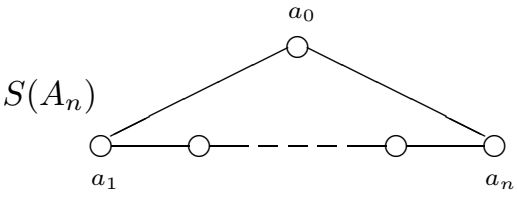

$$
(n \geq 2)
$$

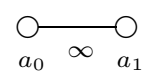

$(n=1)$
(a) $A_{n}^{(1)}$.
(b) $\pm\left(\varepsilon_{i}-\varepsilon_{j}\right)+r c(1 \leq i<j \leq n+1, r \in \mathbb{Z})$.
(c) $a_{0}=-\varepsilon_{1}+\varepsilon_{n}+c, \quad a_{i}=\varepsilon_{i}-\varepsilon_{i+1}(1 \leq i \leq n)$.

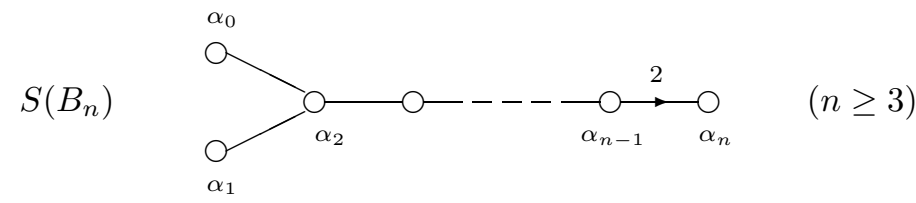
(a) $B_{n}^{(1)}$.
(b) $\pm \varepsilon_{i}+r c(1 \leq i \leq n, r \in \mathbb{Z}) ; \quad \pm \varepsilon_{i} \pm \varepsilon_{j}+r c(1 \leq i<j \leq n, r \in \mathbb{Z})$.
(c) $a_{0}=-\varepsilon_{1}-\varepsilon_{2}+c, \quad a_{i}=\varepsilon_{i}-\varepsilon_{i+1}(1 \leq i \leq n-1), \quad a_{n}=\varepsilon_{n}$.

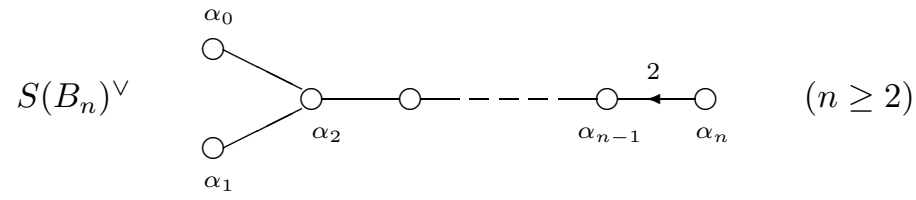
(a) $A_{2 n-1}^{(2)}$.
(b) $\pm 2 \varepsilon_{i}+2 r c(1 \leq i \leq n, r \in \mathbb{Z}) ; \quad \pm \varepsilon_{i} \pm \varepsilon_{j}+r c(1 \leq i<j \leq n, r \in \mathbb{Z})$.
(c) $a_{0}=-\varepsilon_{1}-\varepsilon_{2}+c, \quad a_{i}=\varepsilon_{i}-\varepsilon_{i+1}(1 \leq i \leq n-1), \quad a_{n}=2 \varepsilon_{n}$.

$S\left(C_{n}\right)$

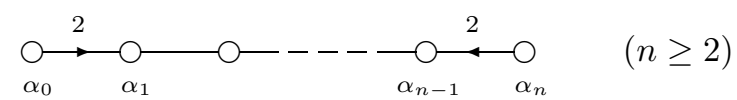
(a) $C_{n}^{(1)}$.
(b) $\pm 2 \varepsilon_{i}+r c(1 \leq i \leq n, r \in \mathbb{Z}) ; \quad \pm \varepsilon_{i} \pm \varepsilon_{j}+r c(1 \leq i<j \leq n, r \in \mathbb{Z})$.
(c) $a_{0}=-2 \varepsilon_{1}+c, \quad a_{i}=\varepsilon_{i}-\varepsilon_{i+1}(1 \leq i \leq n-1), \quad a_{n}=2 \varepsilon_{n}$. 
$S\left(C_{n}\right)^{\vee}$

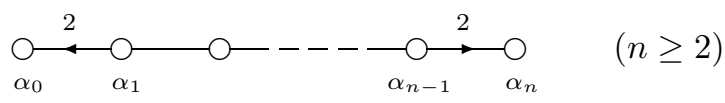

(a) $D_{n+1}^{(2)}$.

(b) $\pm \varepsilon_{i}+\frac{1}{2} r c(1 \leq i \leq n, r \in \mathbb{Z}) ; \quad \pm \varepsilon_{i} \pm \varepsilon_{j}+r c(1 \leq i<j \leq n, r \in \mathbb{Z})$.

(c) $a_{0}=-\varepsilon_{1}+\frac{1}{2} c, \quad a_{i}=\varepsilon_{i}-\varepsilon_{i+1}(1 \leq i \leq n-1), \quad a_{n}=\varepsilon_{n}$.

$S\left(B C_{n}\right)$
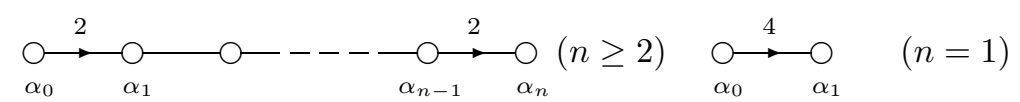

(a) $A_{2 n}^{(2)}$.

(b) $\pm \varepsilon_{i}+r c(1 \leq i \leq n, r \in \mathbb{Z}) ; \quad \pm 2 \varepsilon_{i}+(2 r+1) c(1 \leq i \leq n, r \in \mathbb{Z})$; $\pm \varepsilon_{i} \pm \varepsilon_{j}+r c(1 \leq i<j \leq n, r \in \mathbb{Z})$.

(c) $a_{0}=-2 \varepsilon_{1}+c, \quad a_{i}=\varepsilon_{i}-\varepsilon_{i+1}(1 \leq i \leq n-1), \quad a_{n}=\varepsilon_{n}$.

$S\left(D_{n}\right)$

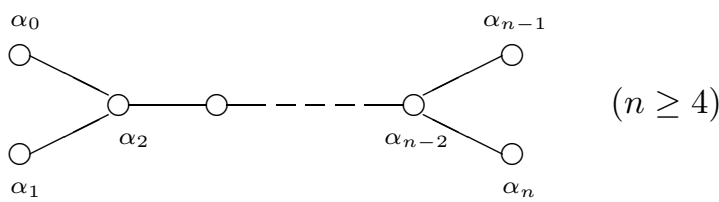

(a) $D_{n}^{(1)}$.

(b) $\pm \varepsilon_{i} \pm \varepsilon_{j}+r c(1 \leq i<j \leq n, r \in \mathbb{Z})$.

(c) $a_{0}=-\varepsilon_{1}-\varepsilon_{2}+c, \quad a_{i}=\varepsilon_{i}-\varepsilon_{i+1}(1 \leq i \leq n-1), \quad a_{n}=\varepsilon_{n-1}+\varepsilon_{n}$.

In the following three cases, let

$$
\omega_{i}=\varepsilon_{i}-\frac{1}{9}\left(\varepsilon_{1}+\cdots+\varepsilon_{9}\right) \quad(1 \leq i \leq 9) .
$$

$S\left(E_{6}\right)$

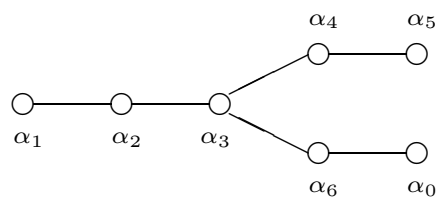

(a) $E_{6}^{(1)}$. 
(b) $\pm\left(\omega_{i}-\omega_{j}\right)+r c(1 \leq i<j \leq 6, r \in \mathbb{Z})$;

$\pm\left(\omega_{i}+\omega_{j}+\omega_{k}\right)+r c(1 \leq i<j<k \leq 6, r \in \mathbb{Z}) ;$

$\pm\left(\omega_{i}+\omega_{2}+\cdots+\omega_{6}\right)+r c(r \in \mathbb{Z})$.

(c) $a_{0}=-\left(\omega_{1}+\cdots+\omega_{6}\right)+c, \quad a_{i}=\omega_{i}-\omega_{i+1}(1 \leq i \leq 5), \quad a_{6}=\omega_{4}+\omega_{5}+\omega_{6}$.

$S\left(E_{7}\right)$

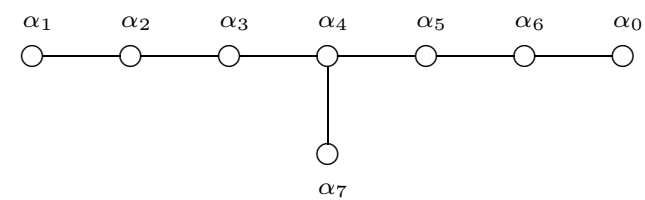
(a) $E_{7}^{(1)}$.
(b) $\pm\left(\omega_{i}-\omega_{j}\right)+r c(1 \leq i<j \leq 7, r \in \mathbb{Z})$;
$\pm\left(\omega_{i}+\omega_{j}+\omega_{k}\right)+r c(1 \leq i<j<k \leq 7, r \in \mathbb{Z})$
$\pm\left(\omega_{1}+\cdots+\hat{\omega}_{i}+\cdots+\omega_{6}\right)+r c(1 \leq i \leq 7, r \in \mathbb{Z})$.
(c) $a_{0}=-\left(\omega_{1}+\cdots+\omega_{7}\right)+c, \quad a_{i}=\omega_{i}-\omega_{i+1}(1 \leq i \leq 6), \quad a_{7}=\omega_{5}+\omega_{6}+\omega_{7}$.

$S\left(E_{8}\right)$

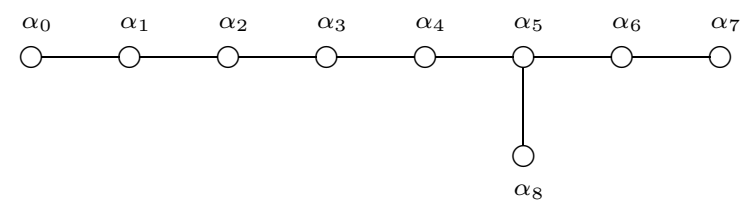
(a) $E_{8}^{(1)}$.
(b) $\pm\left(\omega_{i}-\omega_{j}\right)+r c(1 \leq i<j \leq 9, r \in \mathbb{Z})$;
$\pm\left(\omega_{i}+\omega_{j}+\omega_{k}\right)+r c(1 \leq i<j<k \leq 9, r \in \mathbb{Z}) ;$
(c) $a_{0}=\left(\omega_{1}-\omega_{2}\right)+c, \quad a_{i}=\omega_{i+1}-\omega_{i+2}(1 \leq i \leq 7), \quad a_{8}=\omega_{7}+\omega_{8}+\omega_{9}$.

$S\left(F_{4}\right)$

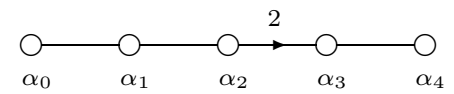
(a) $F_{4}^{(1)}$.
(b) $\pm \varepsilon_{i}+r c(1 \leq i \leq 4, r \in \mathbb{Z}) ; \quad \pm \varepsilon_{i} \pm \varepsilon_{j}+r c(1 \leq i<j \leq 4, r \in \mathbb{Z})$;
$\frac{1}{2}\left( \pm \varepsilon_{1}+ \pm \varepsilon_{2}+ \pm \varepsilon_{3}+ \pm \varepsilon_{4}\right)+r c(r \in \mathbb{Z})$.
(c) $a_{0}=-\varepsilon_{1}-\varepsilon_{2}+c, \quad a_{1}=\varepsilon_{2}-\varepsilon_{3}, \quad a_{2}=\varepsilon_{3}-\varepsilon_{4}, \quad a_{3}=\varepsilon_{4}$,
$a_{4}=\frac{1}{2}\left(\varepsilon_{1}-\varepsilon_{2}-\varepsilon_{3}-\varepsilon_{4}\right)$. 
$S\left(F_{4}\right)^{\vee}$

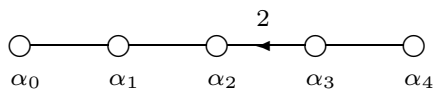

(a) $E_{6}^{(2)}$.

(b) $\pm 2 \varepsilon_{i}+2 r c(1 \leq i \leq 4, r \in \mathbb{Z}) ; \quad \pm \varepsilon_{i} \pm \varepsilon_{j}+r c(1 \leq i<j \leq 4, r \in \mathbb{Z})$; $\pm \varepsilon_{1}+ \pm \varepsilon_{2}+ \pm \varepsilon_{3}+ \pm \varepsilon_{4}+2 r c(r \in \mathbb{Z})$.

(c) $a_{0}=-\varepsilon_{1}-\varepsilon_{2}+c, \quad a_{1}=\varepsilon_{2}-\varepsilon_{3}, \quad a_{2}=\varepsilon_{3}-\varepsilon_{4}, \quad a_{3}=2 \varepsilon_{4}$, $a_{4}=\varepsilon_{1}-\varepsilon_{2}-\varepsilon_{3}-\varepsilon_{4}$.

$S\left(G_{2}\right)$

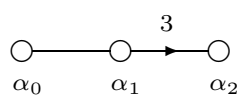

(a) $G_{2}^{(1)}$.

(b) $\pm\left(\varepsilon_{i}-\frac{1}{3}\left(\varepsilon_{1}+\varepsilon_{2}+\varepsilon_{3}\right)\right)+r c(1 \leq i \leq 3, r \in \mathbb{Z})$; $\pm\left(\varepsilon_{i}-\varepsilon_{j}\right)+r c(1 \leq i<j \leq 3, r \in \mathbb{Z})$.

(c) $a_{0}=\varepsilon_{1}-\varepsilon_{2}+c, \quad a_{1}=\varepsilon_{2}-\varepsilon_{3}, \quad a_{2}=\varepsilon_{3}-\frac{1}{3}\left(\varepsilon_{1}+\varepsilon_{2}+\varepsilon_{3}\right)$.

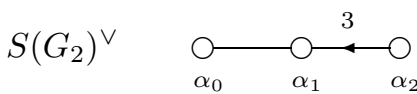

(a) $D_{4}^{(3)}$.

(b) $\pm\left(3 \varepsilon_{i}-\left(\varepsilon_{1}+\varepsilon_{2}+\varepsilon_{3}\right)\right)+3 r c(1 \leq i \leq 3, r \in \mathbb{Z})$; $\pm\left(\varepsilon_{i}-\varepsilon_{j}\right)+r c(1 \leq i<j \leq 3, r \in \mathbb{Z})$.

(c) $a_{0}=\varepsilon_{1}-\varepsilon_{2}+c, \quad a_{1}=\varepsilon_{2}-\varepsilon_{3}, \quad a_{2}=3 \varepsilon_{3}-\left(\varepsilon_{1}+\varepsilon_{2}+\varepsilon_{3}\right)$.

\section{Appendix B. Non-Reduced Affine Root System of Type $\left(C_{n}^{\vee}, C_{n}\right)$}

The non-reduced affine root system of type $\left(C_{n}^{\vee}, C_{n}\right)(n \geq 1)$ is realized as follows:

roots: $\pm \varepsilon_{i}+\frac{1}{2} r c, \quad \pm 2 \varepsilon_{i}+r c(1 \leq i \leq n, r \in \mathbb{Z})$

$\pm \varepsilon_{i} \pm \varepsilon_{j}+r c(1 \leq i<j \leq n, r \in \mathbb{Z})$.

basis: $a_{0}=-\varepsilon_{1}+\frac{1}{2} c, \quad a_{i}=\varepsilon_{i}-\varepsilon_{i+1}(1 \leq i \leq n-1), \quad a_{n}=\varepsilon_{n}$.

Dynkin diagram: 


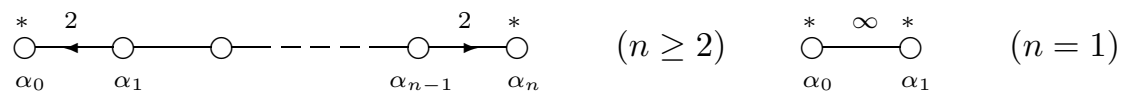

\section{Appendix C. A List of Marked Eliptic Root Systems}

In this paragraph, we will present a complete list of marked elliptic root systems $(R, G)$ with the following assumption,

both $R_{a}=R / G$ and $R_{f}=R / \operatorname{rad} I$ are reduced.

As we mentioned before, they are classified by their elliptic Dynkin diagrams. In the following list, the names of marked elliptic root systems $X^{\left(t_{1}, t_{2}\right)}$ are taken form . For each type, we shall exhibit the elliptic Dynkin diagram and

(a) $\left\{k_{\alpha_{i}}\right\}_{i=0, \cdots, n} ;\left\{m_{i}\right\}_{i=0, \cdots, n}$,

(b) all roots,

(c) the explicit form of $\alpha_{0}, \cdots, \alpha_{n}$,

(d) $R_{f}, R_{a},(R, G)_{a}$,

(e) the dual marked elliptic root system $\left(R^{\vee}, G\right)$ of $(R, G)$.

$A_{1}^{(1,1)}$

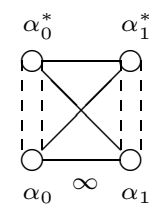

(a) $\quad k_{\alpha_{i}}=1 ; m_{i}=1(i=0,1)$.

(b) $\pm\left(2 \varepsilon_{1}\right)+r \delta_{1}+s \delta_{2}(r, s \in \mathbb{Z})$.

(c) $\alpha_{0}=-2 \varepsilon_{1}+\delta_{2}, \quad \alpha_{1}=2 \varepsilon_{1}$.

(d) $\quad R_{f} \cong A_{1}, R_{a} \cong S\left(A_{1}\right),(R, G)_{a} \cong S\left(A_{1}\right)$.

(e) $\left(A_{1}^{(1,1)}\right)^{\vee}=A_{1}^{(1,1)}$ (self-dual).

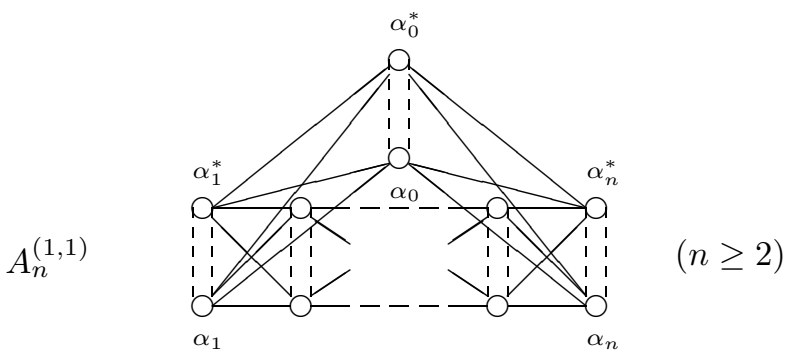

(a) $k_{\alpha_{i}}=1 ; m_{i}=1(0 \leq i \leq n)$.

(b) $\pm\left(\varepsilon_{i}-\varepsilon_{j}\right)+r \delta_{1}+s \delta_{2}(1 \leq i<j \leq n+1, r, s \in \mathbb{Z})$. 
(c) $\alpha_{0}=-\varepsilon_{1}+\varepsilon_{n}+\delta_{2}, \quad \alpha_{i}=\varepsilon_{i}-\varepsilon_{i+1}(1 \leq i \leq n)$.

(d) $R_{f}=A_{n}, R_{a}=S\left(A_{n}\right),(R, G)_{a}=S\left(A_{n}\right)$.

(e) $\left(A_{n}^{(1,1)}\right)^{\vee}=A_{n}^{(1,1)}$ (self-dual).

$A_{1}^{(1,1) *}$

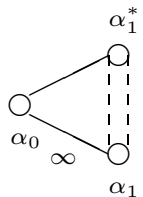

(a) $k_{\alpha_{0}}=2, k_{\alpha_{1}}=1 ; m_{0}=1 / 2, m_{1}=1$.

(b) $\pm \varepsilon_{1}+r \delta_{1}+s \delta_{2}(r, s \in \mathbb{Z}$ such that $r s \equiv 0 \bmod 2)$.

(c) $\alpha_{0}=-\varepsilon_{1}+\delta_{2}, \quad \alpha_{1}=\varepsilon_{1}$.

(d) $R_{f}=A_{1}, R_{a}=S\left(A_{1}\right),(R, G)_{a}=S\left(A_{1}\right)$.

(e) $\left(A_{1}^{(1,1) *}\right)^{\vee}=A_{1}^{(1,1) *}$ (self-dual).

$B_{n}^{(1,1)}$

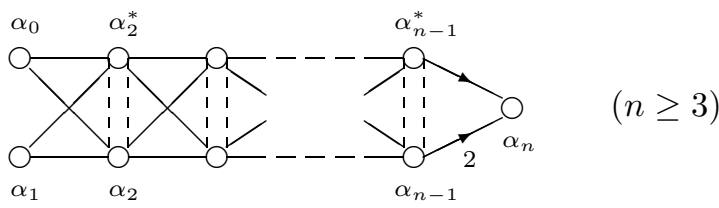

(a) $k_{\alpha_{i}}=1(0 \leq i \leq n) ; m_{0}=2, m_{1}=2, m_{i}=4(2 \leq i \leq n-1)$, $m_{n}=2$.

(b) $\pm \varepsilon_{i}+r \delta_{1}+s \delta_{2}(1 \leq i \leq n+1, r, s \in \mathbb{Z})$, $\pm \varepsilon_{i} \pm \varepsilon_{j}+r \delta_{1}+s \delta_{2}(1 \leq i<j \leq n+1, r, s \in \mathbb{Z})$.

(c) $\alpha_{0}=-\varepsilon_{1}-\varepsilon_{2}+\delta_{2}, \quad \alpha_{i}=\varepsilon_{i}-\varepsilon_{i+1}(1 \leq i \leq n-1), \quad \alpha_{n}=\varepsilon_{n}$.

(d) $R_{f}=B_{n}, R_{a}=S\left(B_{n}\right),(R, G)_{a}=S\left(B_{n}\right)^{\vee}$.

(e) $\left(B_{n}^{(1,1)}\right)^{\vee}=C_{n}^{(2,2)}$.

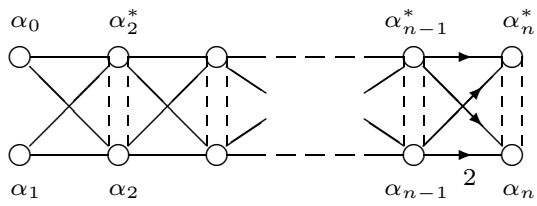

$$
(n \geq 3)
$$

(a) $k_{\alpha_{i}}=2(0 \leq i \leq n-1), k_{\alpha_{n}}=1 ; m_{0}=1, m_{1}=1, m_{i}=2(2 \leq i \leq n)$.

(b) $\pm \varepsilon_{i}+r \delta_{1}+s \delta_{2}(1 \leq i \leq n+1, r, s \in \mathbb{Z})$, 

$\pm \varepsilon_{i} \pm \varepsilon_{j}+2 r \delta_{1}+s \delta_{2}(1 \leq i<j \leq n+1, r, s \in \mathbb{Z})$.
(c) $\alpha_{0}=-\varepsilon_{1}-\varepsilon_{2}+\delta_{2}, \quad \alpha_{i}=\varepsilon_{i}-\varepsilon_{i+1}(1 \leq i \leq n-1), \quad \alpha_{n}=\varepsilon_{n}$.
(d) $R_{f}=B_{n}, R_{a}=S\left(B_{n}\right),(R, G)_{a}=S\left(B_{n}\right)$.
(e) $\left(B_{n}^{(1,2)}\right)^{\vee}=C_{n}^{(2,1)}$.

$B_{n}^{(2,1)}$

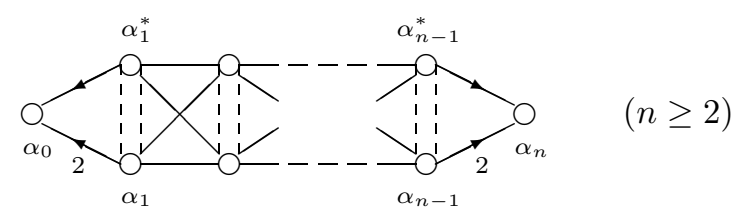

(a) $\quad k_{\alpha_{i}}=1(0 \leq i \leq n) ; m_{0}=1, m_{i}=2(1 \leq i \leq n-1), m_{n}=1$.

(b) $\pm \varepsilon_{i}+r \delta_{1}+s \delta_{2}(1 \leq i \leq n+1, r, s \in \mathbb{Z})$, $\pm \varepsilon_{i} \pm \varepsilon_{j}+r \delta_{1}+2 s \delta_{2}(1 \leq i<j \leq n+1, r, s \in \mathbb{Z})$.

(c) $\alpha_{0}=-\varepsilon_{1}+\delta_{2}, \quad \alpha_{i}=\varepsilon_{i}-\varepsilon_{i+1}(1 \leq i \leq n-1), \quad \alpha_{n}=\varepsilon_{n}$.

(d) $R_{f}=B_{n}, R_{a}=S\left(C_{n}\right)^{\vee},(R, G)_{a}=S\left(C_{n}\right)$.

(e) $\left(B_{n}^{(2,1)}\right)^{\vee}=C_{n}^{(1,2)}$

$B_{n}^{(2,2)}$

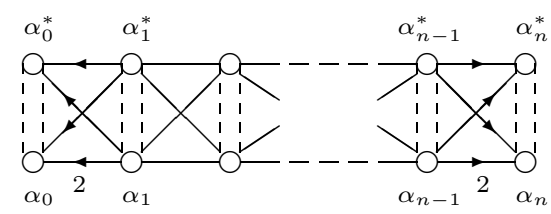

(a) $k_{\alpha_{0}}=1, k_{\alpha_{i}}=2(1 \leq i \leq n-1), k_{\alpha_{n}}=1 ; m_{i}=1(0 \leq i \leq n)$.

(b) $\pm \varepsilon_{i}+r \delta_{1}+s \delta_{2}(1 \leq i \leq n+1, r, s \in \mathbb{Z})$, $\pm \varepsilon_{i} \pm \varepsilon_{j}+2 r \delta_{1}+2 s \delta_{2}(1 \leq i<j \leq n+1, r, s \in \mathbb{Z})$.

(c) $\alpha_{0}=-\varepsilon_{1}+\delta_{2}, \quad \alpha_{i}=\varepsilon_{i}-\varepsilon_{i+1}(1 \leq i \leq n-1), \quad \alpha_{n}=\varepsilon_{n}$.

(d) $R_{f}=B_{n}, R_{a}=S\left(C_{n}\right)^{\vee},(R, G)_{a}=S\left(C_{n}\right)^{\vee}$.

(e) $\left(B_{n}^{(2,2)}\right)^{\vee}=C_{n}^{(1,1)}$.

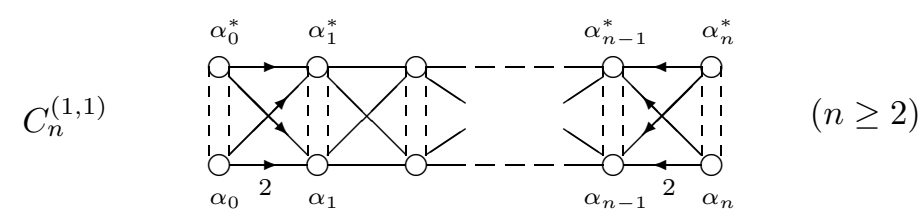

(a) $\quad k_{\alpha_{i}}=1(0 \leq i \leq n) ; m_{i}=2(0 \leq i \leq n)$. 
(b) $\pm 2 \varepsilon_{i}+r \delta_{1}+s \delta_{2}(1 \leq i \leq n+1, r, s \in \mathbb{Z})$, $\pm \varepsilon_{i} \pm \varepsilon_{j}+r \delta_{1}+s \delta_{2}(1 \leq i<j \leq n+1, r, s \in \mathbb{Z})$.

(c) $\alpha_{0}=-2 \varepsilon_{1}+\delta_{2}, \quad \alpha_{i}=\varepsilon_{i}-\varepsilon_{i+1}(1 \leq i \leq n-1), \quad \alpha_{n}=2 \varepsilon_{n}$.

(d) $R_{f}=C_{n}, R_{a}=S\left(C_{n}\right),(R, G)_{a}=S\left(C_{n}\right)^{\vee}$.

(e) $\left(C_{n}^{(1,1)}\right)^{\vee}=B_{n}^{(2,2)}$.

$C_{n}^{(1,2)}$

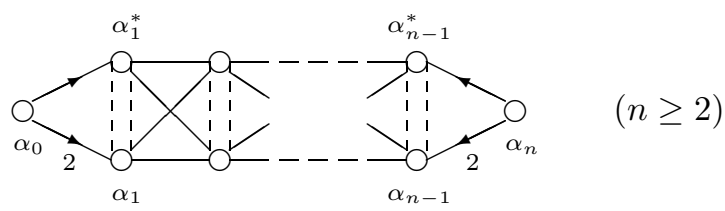

(a) $k_{\alpha_{0}}=2, k_{\alpha_{i}}=1(1 \leq i \leq n-1), k_{\alpha_{n}}=2$;

$m_{0}=1, m_{i}=2(1 \leq i \leq n-1), m_{n}=1$.

(b) $\pm 2 \varepsilon_{i}+2 r \delta_{1}+s \delta_{2}(1 \leq i \leq n+1, r, s \in \mathbb{Z})$,

$\pm \varepsilon_{i} \pm \varepsilon_{j}+r \delta_{1}+s \delta_{2}(1 \leq i<j \leq n+1, r, s \in \mathbb{Z})$.

(c) $\alpha_{0}=-2 \varepsilon_{1}+\delta_{2}, \quad \alpha_{i}=\varepsilon_{i}-\varepsilon_{i+1}(1 \leq i \leq n-1), \quad \alpha_{n}=2 \varepsilon_{n}$.

(d) $R_{f}=C_{n}, R_{a}=S\left(C_{n}\right),(R, G)_{a}=S\left(C_{n}\right)$.

(e) $\left(C_{n}^{(1,2)}\right)^{\vee}=B_{n}^{(2,1)}$.

$C_{n}^{(2,1)}$

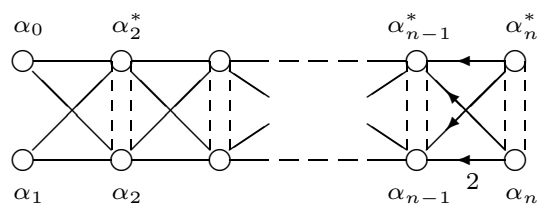

$(n \geq 3)$

(a) $\quad k_{\alpha_{i}}=1(0 \leq i \leq n) ; m_{0}=1, m_{1}=1, m_{i}=2(2 \leq i \leq n)$.

(b) $\pm 2 \varepsilon_{i}+r \delta_{1}+2 s \delta_{2}(1 \leq i \leq n+1, r, s \in \mathbb{Z})$,

$\pm \varepsilon_{i} \pm \varepsilon_{j}+r \delta_{1}+s \delta_{2}(1 \leq i<j \leq n+1, r, s \in \mathbb{Z})$.

(c) $\quad \alpha_{0}=-2 \varepsilon_{1}+\delta_{2}, \quad \alpha_{i}=\varepsilon_{i}-\varepsilon_{i+1}(1 \leq i \leq n-1), \quad \alpha_{n}=2 \varepsilon_{n}$.

(d) $R_{f}=C_{n}, R_{a}=S\left(B_{n}\right)^{\vee},(R, G)_{a}=S\left(B_{n}\right)$.

(e) $\left(C_{n}^{(2,1)}\right)^{\vee}=B_{n}^{(1,2)}$.

$C_{n}^{(2,2)}$

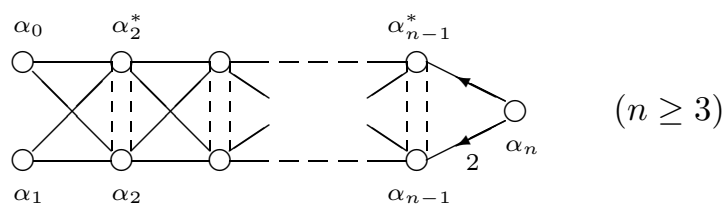



(a) $k_{\alpha_{i}}=1(0 \leq i \leq n-1), k_{\alpha_{n}}=2$;
$m_{0}=1, m_{1}=1, m_{i}=2(2 \leq i \leq n-1), m_{n}=1$.
(b) $\pm 2 \varepsilon_{i}+2 r \delta_{1}+2 s \delta_{2}(1 \leq i \leq n+1, r, s \in \mathbb{Z})$,
$\pm \varepsilon_{i} \pm \varepsilon_{j}+r \delta_{1}+s \delta_{2}(1 \leq i<j \leq n+1, r, s \in \mathbb{Z})$.
(c) $\alpha_{0}=-2 \varepsilon_{1}+\delta_{2}, \quad \alpha_{i}=\varepsilon_{i}-\varepsilon_{i+1}(1 \leq i \leq n-1), \quad \alpha_{n}=2 \varepsilon_{n}$.
(d) $R_{f}=C_{n}, R_{a}=S\left(B_{n}\right)^{\vee},(R, G)_{a}=S\left(B_{n}\right)^{\vee}$.
(e) $\left(C_{n}^{(2,2)}\right)^{\vee}=B_{n}^{(1,1)}$

$B_{n}^{(2,2) *}$

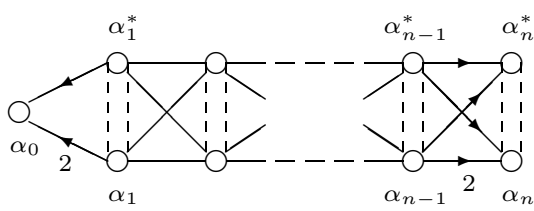

$(n \geq 2)$

(a) $\quad k_{\alpha_{i}}=2(0 \leq i \leq n-1), k_{\alpha_{n}}=1 ; m_{0}=1 / 2, m_{i}=1(1 \leq i \leq n)$.

(b) $\pm \varepsilon_{i}+r \delta_{1}+s \delta_{2}(1 \leq i \leq n+1, r, s \in \mathbb{Z}$ such that $r s \equiv 0 \bmod 2)$, $\pm \varepsilon_{i} \pm \varepsilon_{j}+2 r \delta_{1}+2 s \delta_{2}(1 \leq i<j \leq n+1, r, s \in \mathbb{Z})$.

(c) $\alpha_{0}=-\varepsilon_{1}+\delta_{2}, \quad \alpha_{i}=\varepsilon_{i}-\varepsilon_{i+1}(1 \leq i \leq n-1), \quad \alpha_{n}=\varepsilon_{n}$.

(d) $R_{f}=B_{n}, R_{a}=S\left(C_{n}\right)^{\vee},(R, G)_{a}=S\left(B C_{n}\right)$.

(e) $\left(B_{n}^{(2,2) *}\right)^{\vee}=C_{n}^{(1,1) *}$.

$C_{n}^{(1,1) *}$

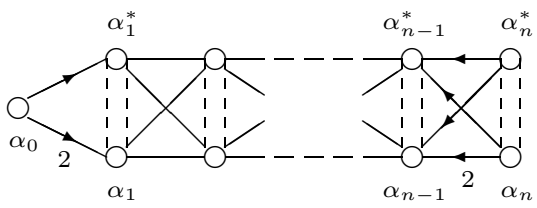

$(n \geq 2)$

(a) $k_{\alpha_{0}}=2, k_{\alpha_{i}}=1(1 \leq i \leq n) ; m_{0}=1, m_{i}=2(1 \leq i \leq n)$.

(b) $\pm 2 \varepsilon_{i}+r \delta_{1}+s \delta_{2}(1 \leq i \leq n+1, r, s \in \mathbb{Z}$ such that $r s \equiv 0 \bmod 2)$, $\pm \varepsilon_{i} \pm \varepsilon_{j}+r \delta_{1}+s \delta_{2}(1 \leq i<j \leq n+1, r, s \in \mathbb{Z})$.

(c) $\alpha_{0}=-2 \varepsilon_{1}+\delta_{2}, \quad \alpha_{i}=\varepsilon_{i}-\varepsilon_{i+1}(1 \leq i \leq n-1), \quad \alpha_{n}=2 \varepsilon_{n}$.

(d) $R_{f}=C_{n}, R_{a}=S\left(C_{n}\right),(R, G)_{a}=S\left(B C_{n}\right)$.

(e) $\left(C_{n}^{(1,1) *}\right)^{\vee}=B_{n}^{(2,2) *}$. 
$D_{n}^{(1,1)}$

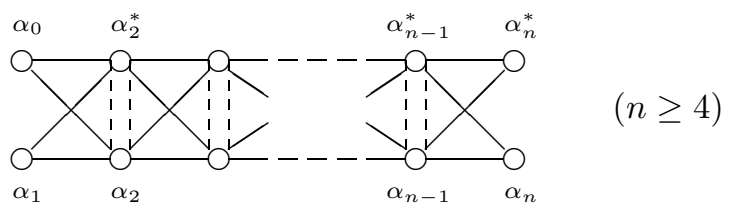

(a) $k_{\alpha_{i}}=1(0 \leq i \leq n)$;

$m_{0}=1, m_{1}=1, m_{i}=2(2 \leq i \leq n-1), m_{n-1}=1, m_{n}=1$.

(b) $\pm \varepsilon_{i} \pm \varepsilon_{j}+r \delta_{1}+s \delta_{2}(1 \leq i<j \leq n+1, r, s \in \mathbb{Z})$.

(c) $\alpha_{0}=-\varepsilon_{1}-\varepsilon_{2}+\delta_{2}, \quad \alpha_{i}=\varepsilon_{i}-\varepsilon_{i+1}(1 \leq i \leq n-1), \quad \alpha_{n}=\varepsilon_{n-1}+\varepsilon_{n}$.

(d) $\quad R_{f}=D_{n}, R_{a}=S\left(D_{n}\right),(R, G)_{a}=S\left(D_{n}\right)$.

(e) $\left(D_{n}^{(1,1)}\right)^{\vee}=D_{n}^{(1,1)}$ (self-dual).

$E_{6}^{(1,1)}$

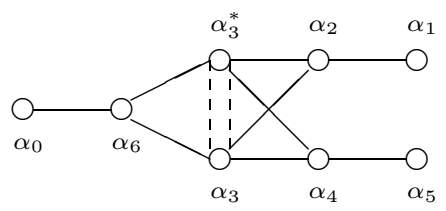

(a) $k_{\alpha_{i}}=1(0 \leq i \leq 6)$;

$m_{0}=1, m_{1}=1, m_{2}=2, m_{3}=3, m_{4}=2, m_{5}=1, m_{6}=2$.

(b) $\pm\left(\omega_{i}-\omega_{j}\right)+r \delta_{1}+s \delta_{2}(1 \leq i<j \leq 6, r, s \in \mathbb{Z})$,

$\pm\left(\omega_{i}+\omega_{j}+\omega_{k}\right)+r \delta_{1}+s \delta_{2}(1 \leq i<j<k \leq 6, r, s \in \mathbb{Z})$,

$\pm\left(\omega_{1}+\omega_{2}+\cdots+\omega_{6}\right)+r \delta_{1}+s \delta_{2}(r, s \in \mathbb{Z})$,

(c) $\alpha_{0}=-\left(\omega_{1}+\cdots+\omega_{6}\right)+\delta_{2}, \quad \alpha_{i}=\omega_{i}-\omega_{i+1}(1 \leq i \leq 5), \quad \alpha_{6}=\omega_{4}+\omega_{5}+\omega_{6}$.

(d) $R_{f}=E_{6}, R_{a}=S\left(E_{6}\right),(R, G)_{a}=S\left(E_{6}\right)$

(e) $\left(E_{6}^{(1,1)}\right)^{\vee}=E_{6}^{(1,1)}$ (self-dual).

$E_{7}^{(1,1)}$

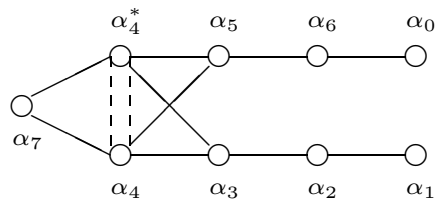

(a) $k_{\alpha_{i}}=1(0 \leq i \leq 7)$;

$m_{0}=1, m_{1}=1, m_{2}=2, m_{3}=3, m_{4}=4, m_{5}=3, m_{6}=2, m_{7}=2$.

(b) $\pm\left(\omega_{i}-\omega_{j}\right)+r \delta_{1}+s \delta_{2}(1 \leq i<j \leq 7, r, s \in \mathbb{Z})$,

$\pm\left(\omega_{i}+\omega_{j}+\omega_{k}\right)+r \delta_{1}+s \delta_{2}(1 \leq i<j<k \leq 7, r, s \in \mathbb{Z})$,

$\pm\left(\omega_{1}+\cdots+\hat{\omega}_{i}+\cdots+\omega_{7}\right)+r \delta_{1}+s \delta_{2}(1 \leq i<j \leq 7, r, s \in \mathbb{Z})$, 
(c) $\alpha_{0}=-\left(\omega_{1}+\cdots+\omega_{6}\right)+\delta_{2}, \quad \alpha_{i}=\omega_{i}-\omega_{i+1}(1 \leq i \leq 6), \quad \alpha_{7}=\omega_{5}+\omega_{6}+\omega_{7}$.

(d) $R_{f}=E_{7}, R_{a}=S\left(E_{7}\right),(R, G)_{a}=S\left(E_{7}\right)$.

(e) $\left(E_{7}^{(1,1)}\right)^{\vee}=E_{7}^{(1,1)}$ (self-dual).

$E_{8}^{(1,1)}$

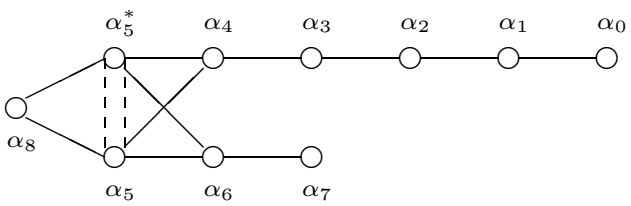

(a) $k_{\alpha_{i}}=1(0 \leq i \leq 8)$

$m_{0}=1, m_{1}=2, m_{2}=3, m_{3}=4, m_{4}=5, m_{5}=6, m_{6}=4, m_{7}=2$,

$m_{8}=3$.

(b) $\pm\left(\omega_{i}-\omega_{j}\right)+r \delta_{1}+s \delta_{2}(1 \leq i<j \leq 9, r, s \in \mathbb{Z})$,

$\pm\left(\omega_{i}+\omega_{j}+\omega_{k}\right)+r \delta_{1}+s \delta_{2}(1 \leq i<j<k \leq 9, r, s \in \mathbb{Z})$,

(c) $\alpha_{0}=\omega_{1}-\omega_{2}+\delta_{2}, \quad \alpha_{i}=\omega_{i+1}-\omega_{i+2}(1 \leq i \leq 7), \quad \alpha_{8}=\omega_{7}+\omega_{8}+\omega_{9}$.

(d) $R_{f}=E_{8}, R_{a}=S\left(E_{8}\right),(R, G)_{a}=S\left(E_{8}\right)$.

(e) $\left(E_{8}^{(1,1)}\right)^{\vee}=E_{8}^{(1,1)}$ (self-dual).

$F_{4}^{(1,1)}$

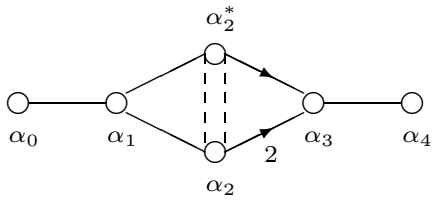

(a) $\quad k_{\alpha_{i}}=1(0 \leq i \leq 4) ; m_{0}=2, m_{1}=4, m_{2}=6, m_{3}=4, m_{4}=2$.

(b) $\pm \varepsilon_{i}+r \delta_{1}+s \delta_{2}(1 \leq i \leq 4, r, s \in \mathbb{Z})$,

$\pm \varepsilon_{i} \pm \varepsilon_{j}+r \delta_{1}+s \delta_{2}(1 \leq i<j \leq 4, r, s \in \mathbb{Z})$,

$\frac{1}{2}\left( \pm \varepsilon_{1}+ \pm \varepsilon_{2}+ \pm \varepsilon_{3}+ \pm \varepsilon_{4}\right)+r \delta_{1}+s \delta_{2}(r, s \in \mathbb{Z})$.

(c) $\alpha_{0}=\varepsilon_{1}-\varepsilon_{2}+\delta_{2}, \quad \alpha_{1}=\varepsilon_{2}-\varepsilon_{3}, \quad \alpha_{2}=\varepsilon_{3}-\varepsilon_{4}, \quad \alpha_{3}=\varepsilon_{4}-\sigma, \quad \alpha_{4}=\sigma$, where $\sigma=\frac{1}{2}\left(\varepsilon_{1}+\varepsilon_{2}+\varepsilon_{3}+\varepsilon_{4}\right)$.

(d) $\quad R_{f}=F_{4}, R_{a}=S\left(F_{4}\right),(R, G)_{a}=S\left(F_{4}\right)^{\vee}$.

(e) $\left(F_{4}^{(1,1)}\right)^{\vee}=F_{4}^{(2,2)}$. 


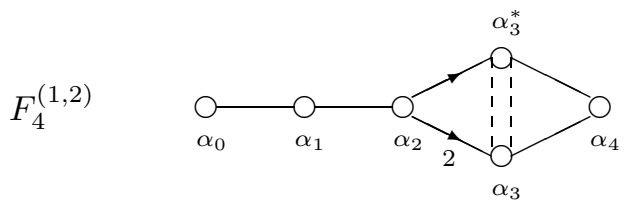

(a) $k_{\alpha_{0}}=2, k_{\alpha_{1}}=2, k_{\alpha_{2}}=2, k_{\alpha_{3}}=1, k_{\alpha_{4}}=1$;

$m_{0}=1, m_{1}=2, m_{2}=3, m_{3}=4, m_{4}=2$.

(b) $\pm \varepsilon_{i}+r \delta_{1}+s \delta_{2}(1 \leq i \leq 4, r, s \in \mathbb{Z})$,

$\pm \varepsilon_{i} \pm \varepsilon_{j}+2 r \delta_{1}+s \delta_{2}(1 \leq i<j \leq 4, r, s \in \mathbb{Z})$,

$\frac{1}{2}\left( \pm \varepsilon_{1}+ \pm \varepsilon_{2}+ \pm \varepsilon_{3}+ \pm \varepsilon_{4}\right)+r \delta_{1}+s \delta_{2}(r, s \in \mathbb{Z})$.

(c) $\alpha_{0}=\varepsilon_{1}-\varepsilon_{2}+\delta_{2}, \quad \alpha_{1}=\varepsilon_{2}-\varepsilon_{3}, \quad \alpha_{2}=\varepsilon_{3}-\varepsilon_{4}, \quad \alpha_{3}=\varepsilon_{4}-\sigma, \quad \alpha_{4}=\sigma$.

(d) $R_{f}=F_{4}, R_{a}=S\left(F_{4}\right),(R, G)_{a}=S\left(F_{4}\right)$.

(e) $\left(F_{4}^{(1,2)}\right)^{\vee}=F_{4}^{(2,1)}$.

$F_{4}^{(2,1)}$

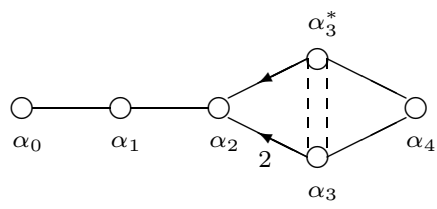

(a) $\quad k_{\alpha_{i}}=1(0 \leq i \leq 4) ; m_{0}=1, m_{1}=2, m_{2}=3, m_{3}=4, m_{4}=2$.

(b) $\pm 2 \varepsilon_{i}+r \delta_{1}+2 s \delta_{2}(1 \leq i \leq 4, r, s \in \mathbb{Z})$,

$\pm \varepsilon_{i} \pm \varepsilon_{j}+r \delta_{1}+s \delta_{2}(1 \leq i<j \leq 4, r, s \in \mathbb{Z})$,

$\pm \varepsilon_{1}+ \pm \varepsilon_{2}+ \pm \varepsilon_{3}+ \pm \varepsilon_{4}+r \delta_{1}+2 s \delta_{2}(r, s \in \mathbb{Z})$.

(c) $\alpha_{0}=\varepsilon_{1}-\varepsilon_{2}+\delta_{2}, \quad \alpha_{1}=\varepsilon_{2}-\varepsilon_{3}, \quad \alpha_{2}=\varepsilon_{3}-\varepsilon_{4}, \quad \alpha_{3}=2 \varepsilon_{4}$,

$\alpha_{4}=-\varepsilon_{1}-\varepsilon_{2}-\varepsilon_{3}-\varepsilon_{4}$.

(d) $R_{f}=F_{4}, R_{a}=S\left(F_{4}\right)^{\vee},(R, G)_{a}=S\left(F_{4}\right)$.

(e) $\left(F_{4}^{(2,1)}\right)^{\vee}=F_{4}^{(1,2)}$.

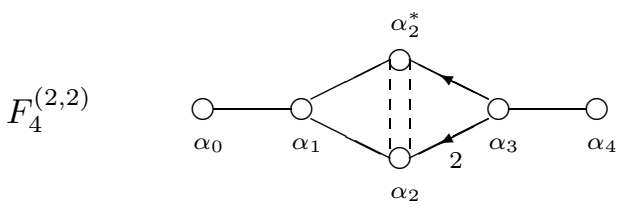

(a) $k_{\alpha_{0}}=1, k_{\alpha_{1}}=1, k_{\alpha_{2}}=1, k_{\alpha_{3}}=2, k_{\alpha_{4}}=2$;

$m_{0}=1, m_{1}=2, m_{2}=3, m_{3}=2, m_{4}=1$.

(b) $\pm 2 \varepsilon_{i}+2 r \delta_{1}+2 s \delta_{2}(1 \leq i \leq 4, r, s \in \mathbb{Z})$, 


$$
\begin{array}{ll} 
& \pm \varepsilon_{i} \pm \varepsilon_{j}+r \delta_{1}+s \delta_{2}(1 \leq i<j \leq 4, r, s \in \mathbb{Z}), \\
& \pm \varepsilon_{1}+ \pm \varepsilon_{2}+ \pm \varepsilon_{3}+ \pm \varepsilon_{4}+2 r \delta_{1}+2 s \delta_{2}(r, s \in \mathbb{Z}) . \\
\text { (c) } & \alpha_{0}=\varepsilon_{1}-\varepsilon_{2}+\delta_{2}, \quad \alpha_{1}=\varepsilon_{2}-\varepsilon_{3}, \quad \alpha_{2}=\varepsilon_{3}-\varepsilon_{4}, \quad \alpha_{3}=2 \varepsilon_{4}, \\
& \alpha_{4}=-\varepsilon_{1}-\varepsilon_{2}-\varepsilon_{3}-\varepsilon_{4} . \\
\text { (d) } & R_{f}=F_{4}, R_{a}=S\left(F_{4}\right)^{\vee},(R, G)_{a}=S\left(F_{4}\right)^{\vee} . \\
\text { (e) } & \left(F_{4}^{(2,2)}\right)^{\vee}=F_{4}^{(1,1)} .
\end{array}
$$

$G_{2}^{(1,1)}$

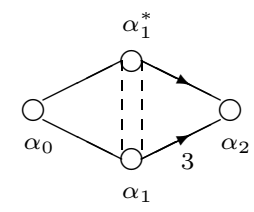

(a) $k_{\alpha_{i}}=1(0 \leq i \leq 2) ; m_{0}=3, m_{1}=6, m_{2}=3$.

(b) $\pm\left(\varepsilon_{i}-\frac{1}{3}\left(\varepsilon_{1}+\varepsilon_{2}+\varepsilon_{3}\right)\right)+r \delta_{1}+s \delta_{2}(1 \leq i \leq 3, r, s \in \mathbb{Z})$, $\pm\left(\varepsilon_{i}-\varepsilon_{j}\right)+r \delta_{1}+s \delta_{2}(1 \leq i<j \leq 3, r, s \in \mathbb{Z})$.

(c) $\alpha_{0}=-\varepsilon_{1}+\varepsilon_{3}+\delta_{2}, \quad \alpha_{1}=\varepsilon_{1}-\varepsilon_{2}, \quad \alpha_{2}=\varepsilon_{2}-\frac{1}{3}\left(\varepsilon_{1}+\varepsilon_{2}+\varepsilon_{3}\right)$.

(d) $R_{f}=G_{2}, R_{a}=S\left(G_{2}\right),(R, G)_{a}=S\left(G_{2}\right)^{\vee}$.

(e) $\left(G_{1}^{(1,1)}\right)^{\vee}=G_{2}^{(3,3)}$.

$G_{2}^{(1,3)}$

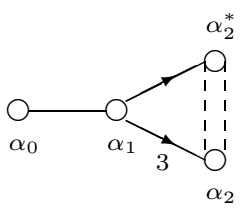

(a) $k_{\alpha_{0}}=3, k_{\alpha_{1}}=3, k_{\alpha_{2}}=1 ; m_{0}=1, m_{1}=2, m_{2}=3$.

(b) $\pm\left(\varepsilon_{i}-\frac{1}{3}\left(\varepsilon_{1}+\varepsilon_{2}+\varepsilon_{3}\right)\right)+r \delta_{1}+s \delta_{2}(1 \leq i \leq 3, r, s \in \mathbb{Z})$, $\pm\left(\varepsilon_{i}-\varepsilon_{j}\right)+3 r \delta_{1}+s \delta_{2}(1 \leq i<j \leq 3, r, s \in \mathbb{Z})$.

(c) $\alpha_{0}=-\varepsilon_{1}+\varepsilon_{3}+\delta_{2}, \quad \alpha_{1}=\varepsilon_{1}-\varepsilon_{2}, \quad \alpha_{2}=\varepsilon_{2}-\frac{1}{3}\left(\varepsilon_{1}+\varepsilon_{2}+\varepsilon_{3}\right)$.

(d) $R_{f}=G_{2}, R_{a}=S\left(G_{2}\right),(R, G)_{a}=S\left(G_{2}\right)$.

(e) $\left(G_{1}^{(1,3)}\right)^{\vee}=G_{2}^{(3,1)}$.

$G_{2}^{(3,1)}$

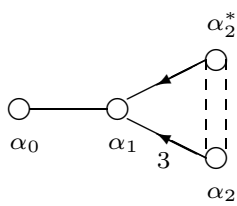


(a) $\quad k_{\alpha_{i}}=1(0 \leq i \leq 2) ; m_{0}=1, m_{1}=2, m_{2}=3$.

(b) $\pm\left(3 \varepsilon_{i}-\left(\varepsilon_{1}+\varepsilon_{2}+\varepsilon_{3}\right)\right)+r \delta_{1}+3 s \delta_{2}(1 \leq i \leq 3, r, s \in \mathbb{Z})$, $\pm\left(\varepsilon_{i}-\varepsilon_{j}\right)+r \delta_{1}+s \delta_{2}(1 \leq i<j \leq 3, r, s \in \mathbb{Z})$.

(c) $\alpha_{0}=-\varepsilon_{1}+\varepsilon_{3}+\delta_{2}, \quad \alpha_{1}=\varepsilon_{1}-\varepsilon_{2}, \quad \alpha_{2}=-\varepsilon_{1}+2 \varepsilon_{2}-\varepsilon_{3}$.

(d) $R_{f}=G_{2}, R_{a}=S\left(G_{2}\right)^{\vee},(R, G)_{a}=S\left(G_{2}\right)$.

(e) $\left(G_{1}^{(3,1)}\right)^{\vee}=G_{2}^{(1,3)}$.

$G_{2}^{(3,3)}$

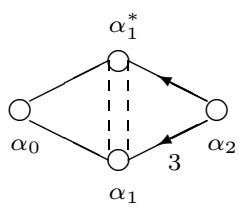

(a) $k_{\alpha_{0}}=1, k_{\alpha_{1}}=1, k_{\alpha_{2}}=3 ; m_{0}=1, m_{1}=2, m_{2}=1$.

(b) $\pm\left(3 \varepsilon_{i}-\left(\varepsilon_{1}+\varepsilon_{2}+\varepsilon_{3}\right)\right)+3 r \delta_{1}+3 s \delta_{2}(1 \leq i \leq 3, r, s \in \mathbb{Z})$, $\pm\left(\varepsilon_{i}-\varepsilon_{j}\right)+r \delta_{1}+s \delta_{2}(1 \leq i<j \leq 3, r, s \in \mathbb{Z})$.

(c) $\alpha_{0}=-\varepsilon_{1}+\varepsilon_{3}+\delta_{2}, \quad \alpha_{1}=\varepsilon_{1}-\varepsilon_{2}, \quad \alpha_{2}=-\varepsilon_{1}+2 \varepsilon_{2}-\varepsilon_{3}$.

(d) $R_{f}=G_{2}, R_{a}=S\left(G_{2}\right)^{\vee},(R, G)_{a}=S\left(G_{2}\right)^{\vee}$.

(e) $\left(G_{1}^{(3,3)}\right)^{\vee}=G_{2}^{(1,1)}$.

\section{Appendix D. The Triplet Associated with $(R, G)$ and the Corresponding Double Affine Hecke Algebra}

We will present a list of the triplet associated with a marked elliptic root system $(R, G)$ and the corresponding double affine Hecke algebra. In the following table, we shall exhibit;

(a) the corresponding triplet and its type;

(b) the type of corresponding double affine Hecke algebra in the sense of [M5].

As we proved in Section 6 , for a triplet of type I or II, $\tilde{\mathcal{A}}_{a} \otimes_{\mathbb{A}} \mathbb{H}(R, G)$ is isomorphic to the small double affine Hecke algebra $\mathcal{H}(\Xi(R, G))_{s}$ and, for a triplet of type III, $\mathbb{H}(R, G)$ is isomorphic to $\mathcal{H}(\Upsilon(R, G))$. In the following table, $\times$ means that we do not consider the corresponding triplet. 


\begin{tabular}{|c|c|c|}
\hline Type of $(R, G)$ & (a) & (b) \\
\hline \multirow[t]{2}{*}{$A_{1}^{(1,1)}$} & $\begin{aligned} \Xi(R, G)= & \left(A_{1} ; S\left(A_{1}\right), Q\left(S\left(A_{1}\right)\right)\right) \\
& (\text { type I or II) }\end{aligned}$ & $\left(S\left(A_{1}\right), S\left(A_{1}\right)\right)$ \\
\hline & $\begin{array}{c}\Upsilon(R, G)=\left(A_{1} ; S\left(A_{1}\right) \cup S\left(A_{1}\right)^{\vee}, Q\left(S\left(A_{1}\right)\right)\right) \\
(\text { type III })\end{array}$ & $\left(C_{1}^{\vee}, C_{1}\right)$ \\
\hline$A_{1}^{(1,1) *}$ & $x$ & $x$ \\
\hline$A_{n}^{(1,1)}(n \geq 2)$ & $\begin{aligned} \Xi(R, G)= & \left(A_{n} ; S\left(A_{n}\right), Q\left(S\left(A_{n}\right)\right)\right) \\
& (\text { type I or II })\end{aligned}$ & $\left(S\left(A_{n}\right), S\left(A_{n}\right)\right)$ \\
\hline$B_{n}^{(1,1)}(n \geq 3)$ & $\begin{array}{c}\Xi(R, G)=\left(B_{n} ; S\left(B_{n}\right), Q\left(S\left(B_{n}\right)^{\vee}\right)\right) \\
(\text { type II })\end{array}$ & $\left(S\left(B_{n}\right), S\left(B_{n}\right)^{\vee}\right)$ \\
\hline$B_{n}^{(1,2)}(n \geq 3)$ & $\begin{array}{c}\Xi(R, G)=\left(B_{n} ; S\left(B_{n}\right), Q\left(S\left(B_{n}\right)\right)\right) \\
\text { (type I) }\end{array}$ & $\left(S\left(B_{n}\right), S\left(B_{n}\right)\right)$ \\
\hline$B_{n}^{(2,1)}(n \geq 2)$ & $\begin{array}{c}\Xi(R, G)=\left(C_{n} ; S\left(C_{n}\right)^{\vee}, Q\left(S\left(C_{n}\right)\right)\right) \\
(\text { type I) }\end{array}$ & $\left(S\left(C_{n}\right)^{\vee}, S\left(C_{n}\right)\right)$ \\
\hline \multirow[t]{2}{*}{$B_{n}^{(2,2)}(n \geq 2)$} & $\begin{array}{c}\Xi(R, G)=\left(C_{n} ; S\left(C_{n}\right)^{\vee}, Q\left(S\left(C_{n}\right)^{\vee}\right)\right) \\
\text { (type II })\end{array}$ & $\left(S\left(C_{n}\right)^{\vee}, S\left(C_{n}\right)^{\vee}\right)$ \\
\hline & $\begin{array}{c}\Upsilon(R, G)=\left(C_{n} ; S\left(C_{n}\right)^{\vee} \cup S\left(C_{n}\right), Q\left(S\left(C_{n}\right)^{\vee}\right)\right) \\
\text { (type III) }\end{array}$ & $\left(C_{n}^{\vee}, C_{n}\right)$ \\
\hline \multirow[t]{2}{*}{$C_{n}^{(1,1)}(n \geq 2)$} & $\begin{array}{c}\Xi(R, G)=\left(C_{n} ; S\left(C_{n}\right), Q\left(S\left(C_{n}\right)^{\vee}\right)\right) \\
\text { (type II) }\end{array}$ & $\left(S\left(C_{n}\right), S\left(C_{n}\right)^{\vee}\right)$ \\
\hline & $\begin{array}{c}\Upsilon(R, G)=\left(C_{n} ; S\left(C_{n}\right) \cup S\left(C_{n}\right)^{\vee}, Q\left(S\left(C_{n}\right)^{\vee}\right)\right) \\
\text { (type III) }\end{array}$ & $\left(C_{n}^{\vee}, C_{n}\right)$ \\
\hline$C_{n}^{(1,2)}(n \geq 2)$ & $\begin{array}{c}\Xi(R, G)=\left(\begin{array}{c}\left(C_{n} ; S\left(C_{n}\right), Q\left(S\left(C_{n}\right)\right)\right) \\
(\text { type I) }\end{array}\right. \\
\end{array}$ & $\left(S\left(C_{n}\right), S\left(C_{n}\right)\right)$ \\
\hline$C_{n}^{(2,1)}(n \geq 3)$ & $\begin{array}{c}\Xi(R, G)=\left(B_{n} ; S\left(B_{n}\right)^{\vee}, Q\left(S\left(B_{n}\right)\right)\right) \\
(\text { type I) }\end{array}$ & $\left(S\left(B_{n}\right)^{\vee}, S\left(B_{n}\right)\right)$ \\
\hline$C_{n}^{(2,2)}(n \geq 3)$ & $\begin{array}{c}\Xi(R, G)=\left(B_{n} ; S\left(B_{n}\right)^{\vee}, Q\left(S\left(B_{n}\right)^{\vee}\right)\right) \\
(\text { type II) }\end{array}$ & $\left(S\left(B_{n}\right)^{\vee}, S\left(B_{n}\right)^{\vee}\right)$ \\
\hline$B_{n}^{(2,2) *}(n \geq 2)$ & $x$ & $x$ \\
\hline$C_{1}^{(1,1) *}(n \geq 2)$ & $\times$ & $\times$ \\
\hline$D_{n}^{(1,1)}(n \geq 4)$ & $\begin{aligned} \Xi(R, G)= & \left(D_{n} ; S\left(D_{n}\right), Q\left(S\left(D_{n}\right)\right)\right) \\
& (\text { type I or II })\end{aligned}$ & $\left(S\left(D_{n}\right), S\left(D_{n}\right)\right)$ \\
\hline$E_{n}^{(1,1)}(n=6,7,8)$ & $\begin{aligned} \Xi(R, G)= & \left(E_{n} ; S\left(E_{n}\right), Q\left(S\left(E_{n}\right)\right)\right) \\
& (\text { type I or II })\end{aligned}$ & $\left(S\left(E_{n}\right), S\left(E_{n}\right)\right)$ \\
\hline$F_{4}^{(1,1)}$ & $\begin{array}{c}\Xi(R, G)=\left(F_{4} ; S\left(F_{4}\right), Q\left(S\left(F_{4}\right)^{\vee}\right)\right) \\
\text { (type II) }\end{array}$ & $\left(S\left(F_{4}\right), S\left(F_{4}\right)^{\vee}\right)$ \\
\hline$F_{4}^{(1,2)}$ & $\begin{array}{c}\Xi(R, G)=\left(F_{4} ; S\left(F_{4}\right), Q\left(S\left(F_{4}\right)\right)\right) \\
(\text { type I })\end{array}$ & $\left(S\left(F_{4}\right), S\left(F_{4}\right)\right)$ \\
\hline$F_{4}^{(2,1)}$ & $\begin{array}{c}\Xi(R, G)=\left(F_{4} ; S\left(F_{4}\right)^{\vee}, Q\left(S\left(F_{4}\right)\right)\right) \\
\text { (type II) }\end{array}$ & $\left(S\left(F_{4}\right)^{\vee}, S\left(F_{4}\right)\right)$ \\
\hline$F_{4}^{(2,2)}$ & $\begin{array}{c}\Xi(R, G)=\left(F_{4} ; S\left(F_{4}\right)^{\vee}, Q\left(S\left(F_{4}\right)^{\vee}\right)\right) \\
(\text { type II) }\end{array}$ & $\left(S\left(F_{4}\right)^{\vee}, S\left(F_{4}\right)^{\vee}\right)$ \\
\hline$G_{2}^{(1,1)}$ & $\begin{array}{c}\Xi(R, G)=\left(G_{2} ; S\left(G_{2}\right), Q\left(S\left(G_{2}\right)^{\vee}\right)\right) \\
\text { (type II) }\end{array}$ & $\left(S\left(G_{2}\right), S\left(G_{2}\right)^{\vee}\right)$ \\
\hline$G_{2}^{(1,3)}$ & $\begin{array}{c}\Xi(R, G)= \\
\quad(\text { type I })\end{array}$ & $\left(S\left(G_{2}\right), S\left(G_{2}\right)\right)$ \\
\hline$G_{2}^{(3,1)}$ & $\begin{array}{c}\Xi(R, G)=\left(G_{2} ; S\left(G_{2}\right)^{\vee}, Q\left(S\left(G_{2}\right)\right)\right) \\
\text { (type I) }\end{array}$ & $\left(S\left(G_{2}\right)^{\vee}, S\left(G_{2}\right)\right)$ \\
\hline$G_{2}^{(3,3)}$ & $\begin{array}{c}\Xi(R, G)=\left(G_{2} ; S\left(G_{2}\right)^{\vee}, Q\left(S\left(G_{2}\right)^{\vee}\right)\right) \\
\text { (type II) }\end{array}$ & $\left(S\left(G_{2}\right)^{\vee}, S\left(G_{2}\right)^{\vee}\right)$ \\
\hline
\end{tabular}


Remark. For a finite root system $R_{0}$ of type $A_{n}, D_{n}, E_{n}, S\left(R_{0}\right)^{\vee}=$ $S\left(R_{0}\right)$. Therefore, for $A_{n}^{(1,1)}, D_{n}^{(1,1)}, E_{n}^{(1,1)}$, we can regard the triplet $\Xi(R, G)$ as of type I or II.

\section{Appendix E. A List of Unequal Parameters}

We will present a list of unequal parameters for an elliptic Hecke algebra $\mathbb{H}(R, G)$ and the corresponding double affine Hecke algebra $\mathcal{H}(\Xi(R, G))$. We remark that, for each case, $\Xi(R, G)$ is a triplet of type I or II.

We shall exhibit in the following table:

(a) the number of unequal parameters in $\mathbb{H}(R, G)$,

(b) independent variables in $\mathbb{A}$,

(c) the number of unequal parameters in $\mathcal{H}(\Xi(R, G))$,

(d) independent variables in $\mathcal{A}$.

\begin{tabular}{|c|c|c|c|c|}
\hline Type of $(R, G)$ & (a) & (b) & (c) & (d) \\
\hline$A_{1}^{(1,1)}$ & 4 & $t_{0}, t_{0^{*}}, t_{1}, t_{1^{*}}$ & 1 & $\tau=\tau_{0}=\tau_{1}$ \\
\hline$A_{1}^{(1,1) *}$ & 3 & $t_{0}, t_{1}, t_{1^{*}}$ & $\times$ & $\times$ \\
\hline$A_{n}^{(1,1)}(n \geq 2)$ & 1 & $\begin{array}{l}t=t_{i}=t_{i^{*}} \\
(0 \leq i \leq n)\end{array}$ & 1 & $\begin{array}{c}\tau=\tau_{i} \\
(0 \leq i \leq n)\end{array}$ \\
\hline$B_{n}^{(1,1)}(n \geq 3)$ & 2 & $\begin{array}{c}t_{n} \\
t=t_{0}=t_{1}=t_{i}=t_{i^{*}} \\
(2 \leq i \leq n-1)\end{array}$ & 2 & $\begin{array}{c}\tau_{n}, \tau=\tau_{i} \\
(0 \leq i \leq n-1)\end{array}$ \\
\hline$B_{n}^{(1,2)}(n \geq 3)$ & 3 & $\begin{array}{c}t_{n}, t_{n^{*}} \\
t=t_{0}=t_{1}=t_{i}=t_{i^{*}} \\
(2 \leq i \leq n-1)\end{array}$ & 2 & $\begin{array}{c}\tau_{n}, \tau=\tau_{i} \\
(0 \leq i \leq n-1)\end{array}$ \\
\hline$B_{n}^{(2,1)}(n \geq 2)$ & 3 & $\begin{array}{c}t_{0}, t_{n}, t=t_{i}=t_{i^{*}} \\
(1 \leq i \leq n-1)\end{array}$ & 2 & $\begin{array}{l}\tau_{0}=\tau_{n}, \tau=\tau_{i} \\
(1 \leq i \leq n-1)\end{array}$ \\
\hline$B_{n}^{(2,2)}(n \geq 2)$ & 5 & $\begin{array}{c}t_{0}, t_{0^{*}}, t_{n}, t_{n^{*}} \\
t=t_{i}=t_{i^{*}} \\
(1 \leq i \leq n-1)\end{array}$ & 2 & $\begin{array}{c}\tau_{0}=\tau_{n}, \tau=\tau_{i} \\
(1 \leq i \leq n-1)\end{array}$ \\
\hline$C_{n}^{(1,1)}(n \geq 2)$ & 5 & $\begin{array}{c}t_{0}, t_{0^{*}}, t_{n}, t_{n^{*}} \\
t=t_{i}=t_{i^{*}} \\
(1 \leq i \leq n-1)\end{array}$ & 2 & $\begin{array}{l}\tau_{0}=\tau_{n}, \tau=\tau_{i} \\
(1 \leq i \leq n-1)\end{array}$ \\
\hline$C_{n}^{(1,2)}(n \geq 2)$ & 3 & $\begin{array}{c}t_{0}, t_{n}, t=t_{i}=t_{i^{*}} \\
(1 \leq i \leq n-1)\end{array}$ & 2 & $\begin{array}{l}\tau_{0}=\tau_{n}, \tau=\tau_{i} \\
(1 \leq i \leq n-1)\end{array}$ \\
\hline$C_{n}^{(2,1)}(n \geq 3)$ & 3 & $\begin{array}{c}t_{n}, t_{n^{*}} \\
t=t_{0}=t_{1}=t_{i}=t_{i^{*}} \\
(2 \leq i \leq n-1)\end{array}$ & 2 & $\begin{array}{c}\tau_{n}, \tau=\tau_{i} \\
(0 \leq i \leq n-1)\end{array}$ \\
\hline
\end{tabular}




\begin{tabular}{|c|c|c|c|c|}
\hline Type of $(R, G)$ & (c) & (d) & (e) & (f) \\
\hline$C_{n}^{(2,2)}(n \geq 3)$ & 2 & $\begin{array}{c}t_{n} \\
t=t_{0}=t_{1}=t_{i}=t_{i^{*}} \\
(2 \leq i \leq n-1)\end{array}$ & 2 & $\begin{array}{c}\tau_{n}, \tau=\tau_{i} \\
(0 \leq i \leq n-1)\end{array}$ \\
\hline$B_{n}^{(2,2) *}(n \geq 2)$ & 4 & $\begin{array}{c}t_{0}, t_{n}, t_{n^{*}}, t=t_{i}=t_{i^{*}} \\
(1 \leq i \leq n-1)\end{array}$ & $\times$ & $\times$ \\
\hline$C_{n}^{(1,1) *}(n \geq 2)$ & 4 & $\begin{array}{c}t_{0}, t_{n}, t_{n^{*}}, t=t_{i}=t_{i^{*}} \\
(1 \leq i \leq n-1)\end{array}$ & $\times$ & $x$ \\
\hline$D_{n}^{(1,1)}(n \geq 4)$ & 1 & $\begin{array}{c}t=t_{\alpha} \\
(\alpha \in \Gamma)\end{array}$ & 1 & $\begin{array}{c}\tau=\tau_{i} \\
(0 \leq i \leq n)\end{array}$ \\
\hline$E_{n}^{(1,1)}(n=6 \sim 8)$ & 1 & $\begin{array}{l}t=t_{\alpha} \\
(\alpha \in \Gamma)\end{array}$ & 1 & $\begin{array}{c}\tau=\tau_{i} \\
(0 \leq i \leq n)\end{array}$ \\
\hline$F_{4}^{(1,1)}$ & 2 & $\begin{array}{c}t_{0}=t_{1}=t_{2}=t_{2^{*}} \\
t_{3}=t_{4}\end{array}$ & 2 & $\begin{array}{c}\tau_{0}=\tau_{1}=\tau_{2} \\
\tau_{3}=\tau_{4}\end{array}$ \\
\hline$F_{4}^{(1,2)}$ & 2 & $\begin{array}{c}t_{0}=t_{1}=t_{2}=t_{3} \\
=t_{3^{*}}, t_{4}\end{array}$ & 2 & $\begin{array}{l}\tau_{0}=\tau_{1}=\tau_{2} \\
=\tau_{3}, \tau_{4}\end{array}$ \\
\hline$F_{4}^{(2,1)}$ & 2 & $\begin{array}{c}t_{0}=t_{1}=t_{2}=t_{3} \\
=t_{3^{*}}, t_{4}\end{array}$ & 2 & $\begin{array}{l}\tau_{0}=\tau_{1}=\tau_{2} \\
\quad=\tau_{3}, \tau_{4}\end{array}$ \\
\hline$F_{4}^{(2,2)}$ & 2 & $\begin{array}{c}t_{0}=t_{1}=t_{2}=t_{2^{*}} \\
t_{3}=t_{4}\end{array}$ & 2 & $\begin{array}{c}\tau_{0}=\tau_{1}=\tau_{2} \\
\tau_{3}=\tau_{4}\end{array}$ \\
\hline$G_{2}^{(1,1)}$ & 2 & $t_{0}=t_{1}=t_{1 *}, t_{2}$ & 2 & $\tau_{0}=\tau_{1}, \tau_{2}$ \\
\hline$G_{2}^{(1,3)}$ & 2 & $t_{0}=t_{1}, t_{2}=t_{2 *}$ & 2 & $\tau_{0}=\tau_{1}, \tau_{2}$ \\
\hline$G_{2}^{(3,1)}$ & 2 & $t_{0}=t_{1}, t_{2}=t_{2^{*}}$ & 2 & $\tau_{0}=\tau_{1}, \tau_{2}$ \\
\hline$G_{2}^{(3,3)}$ & 2 & $t_{0}=t_{1}=t_{1^{*}}, t_{2}$ & 2 & $\tau_{0}=\tau_{1}, \tau_{2}$ \\
\hline
\end{tabular}

Remark. For an elliptic root system of type $A_{1}^{(1,1)}, B_{n}^{(2,2)}$ or $C_{n}^{(1,1)}(n \geq$ 2 ), we consider another double affine Hecke algebra $\mathcal{H}(\Upsilon(R, G))$. The explicit forms of unequal parameters in $\mathcal{H}(\Upsilon(R, G))$ were already given in 6.3.

\section{References}

[AW] R. Askey and J. Wilson, Some basic hypergeometric orthogonal polynomials that generalize Jacobi polynomials, Mem. Amer. Math. Soc. 54 (1985), no. 319, iv+55 pp.

[A] S. Azam, Extended affine root systems, J. Lie Theory 12 (2002), no. 2, 515-527.

[C1] I. Cherednik, Double affine Hecke algebras, Knizhnik-Zamolodchikov equations, and Macdonald's operators, Internat. Math. Res. Notices 1992, no. 9, 171-180.

[C2] , Double affine Hecke algebras and Macdonald's conjectures, Ann. of Math. (2) 141 (1995), no. 1, 191-216. 
[C3] I. Cherednik, Double affine Hecke algebras, London Mathematical Society Lecture Note Series, 319, Cambridge Univ. Press, Cambridge, 2005.

[H] G. W. Hofmann, Weyl groups with Coxeter presentation and presentation by conjugation, J. Lie Theory 17 (2007), no. 2, 337-355.

[K] A. A. Kirillov, Jr., Lectures on affine Hecke algebras and Macdonald's conjectures, Bull. Amer. Math. Soc. (N.S.) 34 (1997), no. 3, 251-292.

[Ko] T. H. Koornwinder, Askey-Wilson polynomials for root systems of type $B C$, in Hypergeometric functions on domains of positivity, Jack polynomials, and applications (Tampa, FL, 1991), 189-204, Contemp. Math., 138, Amer. Math. Soc., Providence, RI, 1992.

[L] H. van der Lek, Extended Artin groups, in Singularities, Part 2 (Arcata, Calif., 1981), 117-121, Proc. Sympos. Pure Math., 40, Amer. Math. Soc., Providence, RI, 1983.

[M1] I. G. Macdonald, Orthogonal polynomials associated with root systems, Sém. Lothar. Combin. 45 (2000/01), Art. B45a, 40 pp. (electronic).

[M2] , Symmetric functions and Hall polynomials, Second edition, Oxford Univ. Press, New York, 1995.

[M3] Affine Hecke algebras and orthogonal polynomials, Astérisque No. 237 (1996), Exp. No. 797, 4, 189-207.

[M4] Symmetric functions and orthogonal polynomials, University Lecture Series, 12, Amer. Math. Soc., Providence, RI, 1998.

[M5] , Affine Hecke algebras and orthogonal polynomials, Cambridge Tracts in Mathematics, 157, Cambridge Univ. Press, Cambridge, 2003.

[N] M. Noumi, Macdonald-Koornwinder polynomials and affine Hecke rings, Sūrikaisekikenkyūsho Kōkyūroku No. 919 (1995), 44-55 (written in Japanese).

[NS] M. Noumi and J. V. Stokman, Askey-Wilson polynomials: an affine Hecke algebra approach, in Laredo Lectures on Orthogonal Polynomials and Special Functions, 111-144, Adv. Theory Spec. Funct. Orthogonal Polynomials, Nova Sci. Publ., Hauppauge, NY, 2004.

[Sa] S. Sahi, Nonsymmetric Koornwinder polynomials and duality, Ann. of Math. (2) 150 (1999), no. 1, 267-282.

[S] K. Saito, Extended affine root systems. I. Coxeter transformations, Publ. Res. Inst. Math. Sci. 21 (1985), no. 1, 75-179.

[ST] K. Saito and T. Takebayashi, Extended affine root systems. III. Elliptic Weyl groups, Publ. Res. Inst. Math. Sci. 33 (1997), no. 2, 301-329.

[SS] Y. Saito and M. Shiota, On Hecke algebras associated to elliptic root systems, to appear in Representation Theory of Algebraic Groups and Quantum Groups, Birkhäuser.

[T1] T. Takebayashi, Double affine Hecke algebras and elliptic Hecke algebras, J. Algebra 253 (2002), no. 2, 314-349.

[T2] , Elliptic Artin groups and elliptic Hecke algebras, J. Algebra 292 (2005), no. 2, 343-362.

[Y] H. Yamada, Elliptic root system and elliptic Artin group, Publ. Res. Inst. Math. Sci. 36 (2000), no. 1, 111-138. 